\title{
Reverse Mathematics of Divisibility in Integral Domains
}

by

Valentin B. Bura

\author{
A thesis \\ submitted to the Victoria University of Wellington \\ in fulfilment of the \\ requirements for the degree of \\ Master of Science \\ in Mathematics. \\ Victoria University of Wellington \\ 2013
}





\begin{abstract}
This thesis establishes new results concerning the proof-theoretic strength of two classic theorems of Ring Theory relating to factorization in integral domains.

The first theorem asserts that if every irreducible is a prime, then every element has at most one decomposition into irreducibles; the second states that well-foundedness of divisibility implies the existence of an irreducible factorization for each element.

After introductions to the Algebra framework used and Reverse Mathematics, we show that the first theorem is provable in the base system of Second Order Arithmetic $R C A_{0}$, while the other is equivalent over $R C A_{0}$ to the system $A C A_{0}$.
\end{abstract}




\section{Acknowledgments}

Foremost thanks are due to my supervisor, A/Professor Noam Greenberg, for suggesting the topic and directing its development. Collaborating with him was a privilege and a first-rate learning opportunity: his great deal of patience, thoughtful advice, pedagogical foresight and mathematical intuition have guided my steps through this first research project.

Professor Rod Downey influenced my journey as a graduate student in Mathematics.

Dr Peter Donelan and Dr BD Kim were available for discussion and had suggestions. Dr Dillon Mayhew supervised my research for a period of time.

Dr David Diamondstone and Dr Dan Turetski, the postdocs working in Computability at SMSOR, have helped the development of my ideas.

I was inspired by many of my past lecturers in Logic, including Professor Cris Calude at Auckland, Dr Colin Bailey, Professor Rob Goldblatt and Professor Edwin Mares in Wellington.

I have benefited from the friendship and fruitful exchange of ideas with Ahmad Abdul-Ghaffar, Valentina Baccetti, Amanda Cameron, Dr Carolyn Chun, Mohammed Daher, Henry Macdonald, Joel Miller and Zheng Jingwei.

My thoughts turn towards the multitude of people from which I have learned during my formative years. I mention here three of my high-school teachers: Mioara Enache and Ioan Lepãdatu, who have fostered my mathematical abilities, and Gilbert Danciu, who has instilled in my mind the desire to succeed in academic research.

On a personal note, I thank my parents, Lucian and Raluca, for their continuous encouragement and their support on multiple levels, key ingredients in my ability to pursue a higher education. 


\section{Contents}

1 Introduction $\quad \mathbf{1}$

1.1 Background . . . . . . . . . . . . . . . . 1

1.2 Effective and Reverse Algebra . . . . . . . . . . . . . 3

1.3 Results ....................... 5

2 Elements of Algebra $\quad 9$

2.1 Divisibility . . . . . . . . . . . . . . . 10

2.2 Localization . . . . . . . . . . . . . . . 15

2.3 Polynomial Rings . . . . . . . . . . . . . . . . . . . . 19

2.4 Independence and Generation . . . . . . . . . . . . . 23

3 Reverse Mathematics and Logic 33

3.1 Notions from Computability Theory . . . . . . . . . . . . . . 34

3.2 Reverse Mathematics . . . . . . . . . . . . . . . . . . . . . . 35

3.3 Subsystems of $\mathcal{Z}_{2} \ldots \ldots \ldots \ldots$

3.4 Computability and Algebra . . . . . . . . . . . 39

4 Equivalent characterizations of UFDs 49

4.1 The theorems . . . . . . . . . . . . . . . . 49

4.2 A tree encoding $\emptyset^{\prime} \ldots \ldots \ldots \ldots \ldots$

4.3 Equivalence with $A C A_{0} \quad \ldots \ldots \ldots$. . . . . . . 58

4.4 Conclusion . . . . . . . . . . . . . . . 79 


\section{Chapter 1}

\section{Introduction}

The program of Reverse Mathematics was introduced by Harvey Friedman in his 1974 address to the International Congress of Mathematicians in Vancouver. It is a program in Mathematical Logic with deep philosophical import, redefining one of the pillars of the Foundations of Mathematics, namely Proof Theory.

\subsection{Background}

Reverse Mathematics is a study of the foundations of ordinary Mathematics, and this involves both reasoning within a given proof system and "going backwards from the theorems to the first principles". The main question it poses is "which set-existence axioms are needed to prove the theorems of non-set-theoretic Mathematics?" It stands in intimate connection with Computability Theory, since a certain notion of effectiveness provides a foundation to these types of investigations.

The results the logician focuses on are, in a sense, already known to be true if one considers the larger proof framework of ZFC Set Theory. It is not the 'truth' of results that one seeks to establish, but the more philosophically adequate notion of 'relative truth'. We come to a better understanding of what this means if we try to restrict our attention to different programs in the Philosophy of Mathematics, ranging from Constructive Mathematics to Impredicativism.

The process is carried out in subsystems of Second Order Arithmetic, 
where one takes a base system as proving grounds and then proves that a certain theorem is equivalent to a stronger set of axioms, over the base system. Like any equivalence, this proof is done in two parts: first, a direct proof is formulated that the theorem follows from the axioms, and then a 'reversal' step is appended, in which it is shown that the axioms follow if we are to assume the theorem.

In the words of S. S. Wainer, reviewing Friedman's survey, two main themes can be identified straight away: first, that "the 'proper' axioms to use in proving particular fundamental theorems of mathematics often turn out to be (provably) equivalent to those theorems", and second that "stronger axioms are needed to provide explicit definitions of hard-todefine sets of integers than merely to prove their existence".

Friedman introduced several axioms for arithmetic sets. RCA, the base system, which is too weak for most proofs but strong enough for most definitions, consists of basic axioms for arithmetic manipulations, a restricted induction scheme and a comprehension scheme for computable properties. Hence, provability in this system is equivalent to effectiveness.

ACA is the Arithmetic Comprehension axiom system, KL consists of RCA (recursive comprehension axioms) plus König's Lemma, SLUB consists of RCA plus the axiom "every bounded sequence of reals has a l.u.b.". SBW is the sequential Bolzano-Weierstrass system. The above systems are all equivalent.

WKL (the weak König's Lemma system, for binary trees) is equivalent to SHB (sequential Heine-Borel system) and to the reflection principle "if a statement is true there is a structure in which it holds" (it has a model), which can also be viewed as a soundness statement. The second theme is illustrated by the fact that whereas ACA is clearly sufficient to explicitly define non-recursive sets, WKL is not.

Even though it deals with Constructivism, Reverse Mathematics differs from this school of thought because it assumes the framework of Classical Logic and does not make the radical ontological commitments of intuitionists.

However, from this perspective, Reverse Mathematics is the answer to those who are not prepared to make the full set-existence commitments that a working mathematician makes: the big five subsystems correspond 
roughly to Bishop's Constructive mathematics, Hilbert's Finitistic reductionism, Weyl's and Feferman's Predicativism, Friedman's and Simpson's Predicative reductionism, and to Impredicativism respectively.

\subsection{Effective and Reverse Algebra}

Effective Algebra incorporates both Computable Algebra and Constructive Algebra. Good surveys of the latter are found in $[1,16]$.

The systematic study of computability in the framework of Ring Theory started in the fifties and sixties with $[7,8,20,21]$.

The work of Fröhlich and Sheperdson in [7,8] assumed a less rigorous foundation for computable sets, while Rabin in [20,21] and Mal'cev in [14] developed the modern concept of a computable structure by using an analogue of the Gödel numbering of logical syntax.

The focus of [7] was effective factorization of polynomials, extending on the work of Van der Waerden ([29]) and Kneser([12]). In particular, [29] has discussed the problem of carrying out certain field theoretical procedures effectively, in a "finite number of steps", and it was shown that there can be no general algorithm for splitting polynomials over an explicitly given field $K$, more precisely that such an algorithm would lead to a general procedure for deciding whether arbitrarily given properties of positive integers are instantiated. (A splitting algorithm for a field $K$ decides the irreducibility of a polynomial over $K[x]$.) In [29] it was defined an "explicitly given" field as one whose elements are uniquely represented by distinguishable symbols with which one can perform the operations of addition, multiplication, subtraction and division in a finite number of steps, and Fröhlich and Sheperdson assume the same framework.

In $[7,8]$ it was constructed an explicit field for which there is no splitting algorithm, and explicit fields $K, \bar{K}$ such that $\bar{K}$ is a simple non separable extension of $K$, and $K$ has a splitting algorithm but $\bar{K}$ does not. In addition to this, they show there exist isomorphic "explicitly given fields", one of which possesses a splitting algorithm but the other does not, and that a computable field with a splitting algorithm has a computable algebraic closure that is unique up to computable isomorphism.

In $[20,21]$ Rabin develops the notion of what it means for an algebraic 
structure to be "computable" by defining an indexing of a set $S$ as an injective mapping $i: S \rightarrow \omega$ such that $i(S)$ is a recursive subset of $\omega$. Several results are then proved, including the fact that the algebraic closure $T$ of any computable field $K$ is computable, and the natural embedding of $K$ into $T$ is computable.

Mal'cev ([14]), working on the same framework based on Gödel numbering, focused on effective universal algebras.

A historical note on Computability in Ring Theory is found in [26].

Reverse Algebra (Reverse Mathematics in the context of Algebra) is intimately related to Computable Algebra and debuted with the seminal paper by Friedman et al. "Countable Algebra and set existence axioms" ([6]). Several equivalences are proved over $R C A_{0}$. It is shown that $W K L_{0}$ is equivalent to the following statements: every countable field has a unique algebraic closure; every countable formally real field is orderable; every countable formally real field has a real closure; every countable commutative ring has a prime ideal. It is shown that $A C A_{0}$, is equivalent to the statements: every countable field is isomorphic to a subfield of its algebraic closure; every countable ordered field is isomorphic to a subfield of its real closure; every countable field has a transcendence base; every countable vector space has a basis; every countable abelian group has a torsion subgroup; every countable abelian group has a unique divisible hull; every countable commutative ring (or countable integral domain) has a maximal ideal. Finally, it is shown that $A T R_{0}$ is equivalent to the statement: every countable reduced abelian group has a system of Ulm invariants which determine it up to isomorphism, and that $\Pi_{1}^{1}-C A$ is equivalent to the statement: every countable abelian group is the direct sum of a divisible group and a reduced group.

Subsequently, weaker theories have been investigated to allow for equivalences with $R C A_{0}$ to be proven; some equivalences are: every finitely generated vector space over a countable field has a basis (Friedman quoted by [11]); a polynomial over a countable field has finitely many roots, such a polynomial has an irreducible factor, the ring of polynomials over a countable field is a unique factorization domain ([25]) and a principal ideal domain, every countable Euclidean domain is a unique factorization domain, if $R$ is a countable unique factorization domain, so is $R[x]$ ([22]).

For a treatment of Reverse Algebra we refer the reader to [24]. 


\subsection{Results}

Our problems relate to two well-known equivalent characterizations for Unique Factorization Domains. Either one of these characterizations is employed as a definition, while the other is showed to be equivalent. The two characterizations are:

ACCP \& AP-domain

Atomic \& U-UFD

Characterization I invokes the Ascending Chain Condition on Principal Ideals (ACCP), which is equivalent to the statement "the divisibility relation is well-founded". It also invokes the property of being an AP-domain, by which every irreducible is a prime. Hence, the first characterization reads:

The divisibility relation is well-founded and every irreducible is a prime.

Characterization II invokes the property of being Atomic, which requires every element of the ring to have an irreducible factorization, and the property of being an U-UFD, which stipulates that any element which admits an irreducible factorization has a unique such factorization, up to units and order of the factors. So this reads:

Every element has exactly one factorization into irreducibles.

The equivalence is not hard to prove, and we state it here as the first theorem:

Theorem 1.1. Let $R$ be an integral domain. The following are equivalent:

1. $R$ is an AP domain which satisfies the ACCP;

2. $R$ is an Atomic U-UFD.

These two characterizations hint towards two other classic theorems of Ring Theory. We will use these two theorems as objects of study and we will provide their strength relative to the five subsystems of Second Order Arithmetic used in Reverse Mathematics. The theorems depict two different implications. The first one links irreducibles, primes and unique factorizations: 
Theorem 1.2. If an integral domain is $A P$, then it is an U-UFD.

As expected, the second theorem links ACCP and Atomicity:

Theorem 1.3. If an integral domain satisfies the ACCP, then it is Atomic.

Given the equivalence between the characterizations and the above implications, it is somewhat surprising that neither of the two converses hold, as shown in [10, 2]. Hence the situation is, in some sense, asymmetrical. This relationship is depicted below by Figure 1.1.

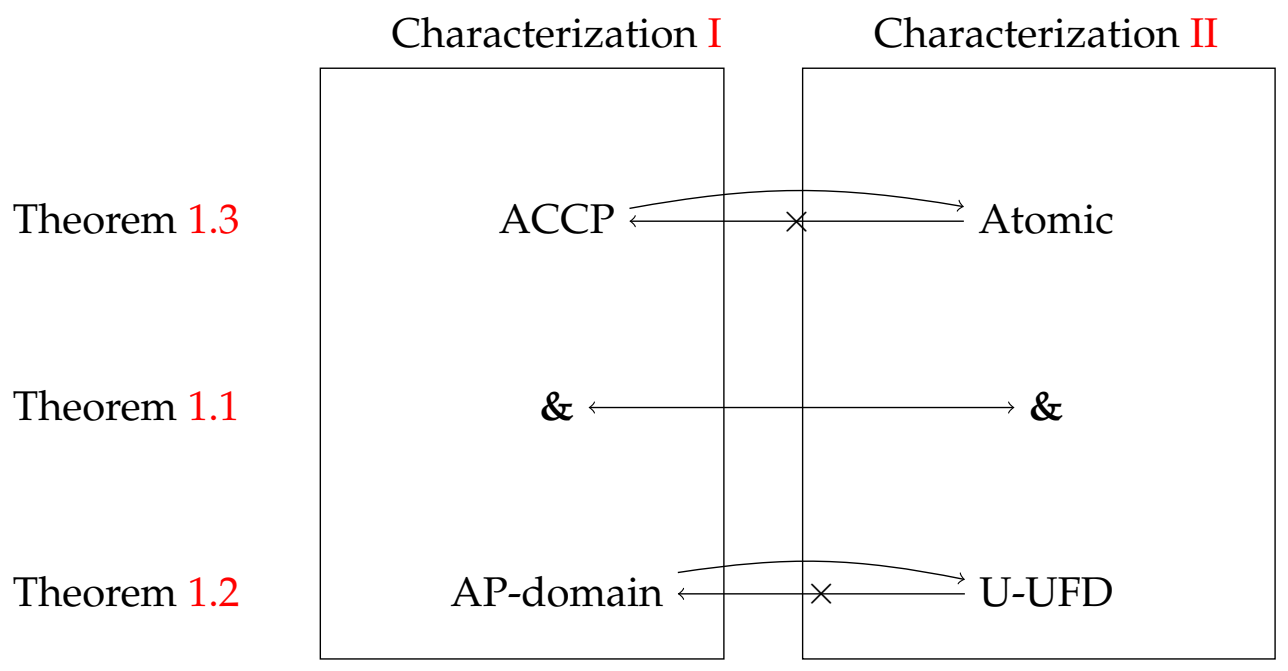

Figure 1.1: The logical structure of our main problems

It is perhaps less surprising that theorems 1.2 and 1.3 exhibit different proof-theoretic strengths. This can be easily seen by examining their standard proofs: while the proof of the second holds effectively, the proof of the first one requires the Halting Set as an oracle. It is these novel facts that the present work establishes.

More precisely, we give an involved proof that theorem 1.3 is equivalent to $A C A_{0}$ over $R C A_{0}$ while also giving an argument that theorem 1.2 is provable in $R C A_{0}$.

Chapter 2 introduces the notions of Algebra we will make use of in our treatment of UFDs. Chapter 3 provides a short introduction to Computability Theory and Reverse Mathematics and presents some results concerning Effective Algebra that will be useful in the next Chapter. Finally, Chapter 4 
focuses on our main results. A brief conclusion is formulated at the end of Chapter 4. 


\section{Chapter 2}

\section{Elements of Algebra}

This chapter sets up the framework of Abstract Algebra we will be interested in, which concerns the theory of commutative rings. Some of the material presented here is standard, but we include it in order to lay a foundation to our inquiry. Other parts of this chapter are more specialized and form prerequisites of Chapter 4 , in which our results are presented.

In the Chapters concerning Algebra the capital letters $P, Q, R, S$ will denote rings, small letters will usually denote elements of rings, maps will be referenced by greek letters $\varphi, \psi, \eta$, sets will be referenced by capitals $I, J, K$ and the letters $f, g, h$ will be reserved for polynomials.

We start by defining a ring structure.

Definition 2.1. A ring is a nonempty set $R$ endowed with two binary operations, + and ·, such that for all $a, b, c \in R$ the following conditions hold:

1. $a+b=b+a$ (commutativity of addition);

2. $(a+b)+c=a+(b+c)$ (associativity of addition);

3. $\exists 0 \in R$ such that $\forall a \in R, a+0=a$ (additive identity);

4. $\forall a \in R \exists-a \in R$ such that $a+(-a)=0$ (additive inverses);

5. $a \cdot b=b \cdot a$ (commutativity of multiplication);

6. $a \cdot(b \cdot c)=(a \cdot b) \cdot c$ (associativity of multiplication);

7. $\exists 1 \in R$ such that $\forall a \in R, a \cdot 1=a$ (multiplicative identity);

8. $a \cdot(b+c)=a \cdot b+a \cdot c$ (distributivity).

In the literature, the structure defined above is known as a "commutative 
ring with unity". These are precisely the kind of rings we will make use of in the present work, so we have restricted the definition of a ring to only include these objects.

Examples include the integers $\mathbb{Z}$, the integers modulo $n, \mathbb{Z} / n \mathbb{Z}$, and the set $Z[x]$ of all polynomials in the variable $x$ with integer coefficients. We will give a formal treatment of polynomial rings below.

We will write $\left(R,+_{R}, \cdot_{R}, 0_{R}, 1_{R}\right)$ for the ring $R$, and if it is understood from context, the subscripts will be dropped.

For the treatment of basic Ring Theory, we follow Gallian in [9].

\subsection{Divisibility}

We begin our treatment of divisibility here.

Definition 2.2. A ring in which there are no non-zero elements $a, b$ such that $a \cdot b=0$ is called an integral domain.

A unit is an element $a \in R$ such that there is $b \in R$ with $a \cdot b=1_{R}$. The set of units of $R$ is denoted as $R^{\times}$. In an integral domain, the element $a$ divides the element $b$ if there exists $c$ such that $a \cdot c=b$. We write $a \mid b$. Elements $a$ and $b$ of an integral domain $D$ are called associates if $a=u \cdot b$ for a unit $u$. This is equivalent to $a \mid b$ and $b \mid a$, and we will write $a \sim b$. A non-zero element $a$ of an integral domain $D$ is called irreducible if it is a non-unit and, whenever $b, c \in D$ with $a=b \cdot c$, then $b$ is a unit or $c$ is a unit. In other words, a is irreducible if a is not a unit and whenever $b \mid a$, either $b$ and $a$ are associates or $b$ is a unit. A non-zero element $a$ of an integral domain $D$ is called a prime if $a$ is not a unit and $a \mid b \cdot c$ implies $a \mid b$ or $b \mid c$. For example, in $\mathbb{Z}$, the irreducible elements are the prime numbers $p$ and their additive inverses $-p$. Non-unit elements which are not irreducible are called reducible.

Proposition 2.3. In an integral domain, every prime is irreducible.

Proof. Suppose $p$ is prime and $p=a \cdot b$. Then, $p \mid a b$, by primeness $p \mid a$ or $p \mid b$. Suppose without loss of generality that $p \mid a$, we have that $p \sim a$, so $b$ must be invertible. 
The converse does not always hold; we will reserve a special name for a ring in which the converse is true.

Definition 2.4. A ring in which every irreducible is prime is called an $A P$ domain.

Definition 2.5. An ideal of a ring $R$ is a non-empty subset $I$ of $R$ such that:

1. $a-b \in I$ whenever $a, b \in I$,

2. $r \cdot a \in I$ whenever $r \in R$ and $a \in I$.

Hence, an ideal generated by $I$ in $R$ has the form:

$$
\langle I\rangle=\left\{r_{1} \cdot a_{1}+r_{2} \cdot a_{2}+\cdots+r_{k} \cdot a_{k} \mid r_{1}, r_{2} \cdots r_{k} \in R, a_{1}, a_{2} \cdots a_{k} \in I\right\} .
$$

A multiset $B$ is a pair $(S, m)$, where $S$ is a set and $m$ is a function $m: S \rightarrow \omega$. If $s \in S, m_{s}(B)=m(s)$ denotes the multiplicity of $s$ in $B$. If $S=\left\{s_{1}, s_{2} \cdots s_{k} \cdots\right\}$ with $m_{s_{i}}=n_{i}$, we write

$$
B=\left[s_{1}, s_{1} \cdots s_{1}, s_{2}, s_{2} \cdots s_{2}, \cdots s_{k}, s_{k} \cdots s_{k}, \cdots\right]
$$

where each $s_{i}$ appears in $B n_{i}$ many times.

To formalize the idea of unique factorization, we use the following definition.

Definition 2.6. An irreducible (prime) factorization of $p$ in the $\operatorname{ring} Q$ is a finite multiset of irreducible (prime) elements $B=\left[p_{i} \mid i \leq n\right]$ in $Q$, such that $p \sim \prod_{i=1}^{n} p_{i}$.

We say $B$ is a subset of $B^{\prime}$ up to association if there exists an injective function $f: B \rightarrow B^{\prime}$ such that if $f(p)=p^{\prime}$, then $p \sim p^{\prime}$. If $f$ is a bijection we say $B$ and $B^{\prime}$ are equal up to association and write $B \sim B^{\prime}$.

Since functions on multisets are defined for each copy of an element of their domain, copies of the same element may be mapped differently. For example, in the ring of integers $[2,2]$ is a subset of $[2,-2,3]$ up to association, since we can put $f(2)=2$ and $f(2)=-2$; the two copies of the integer 2 represent distinct elements of the domain of $f$.

Atomicity will play an important part in the present work. We define it here. 
Definition 2.7. An integral domain $R$ is Atomic if every non-zero element of $R$ has an irreducible factorization.

Note that the factorization of a unit of $R$ is an empty multiset.

U-UFDs are also central in our treatment of divisibility.

Definition 2.8. An integral domain in which every element that admits a factorization into irreducible factors has a unique such factorization (up to association of multisets) is called an unrestricted unique factorization domain, or U-UFD for short.

We are now ready to define a Unique Factorization Domain.

Definition 2.9. An integral domain $R$ is a unique factorization domain if

1. $R$ is Atomic,

2. $R$ is a U-UFD.

A principal ideal in a ring $R$ is an ideal $I$ generated by a single element $a$, i.e. $I=\{r \cdot a \mid r \in R\}$.

A principal ideal domain (PID) is an integral domain in which every ideal is principal. In a PID, the set of irreducibles coincides with the set of primes. Every element of a PID admits a factorization into irreducibles. It turns out that every PID is a UFD, for example the Integers and the Gaussian Integers. It must be noted that the converse does not hold: not every UFD is a PID. For example, $\mathbb{Z}[x]$ is not a PID (e.g. the set of polynomials in $\mathbb{Z}[x]$ whose constant term is even is a non-principal ideal) but it is a UFD.

As in a PID, every irreducible of a UFD is a prime.

Proposition 2.10. Every UFD is an AP domain.

Proof. Let $p$ be an irreducible of $Q$ and suppose $p h=a b$. We have $h=$ $\prod_{h_{i} \in B_{h}} h_{i}, a=\prod_{a_{j} \in B_{a}} a_{j}$ and $b=\prod_{b_{k} \in B_{b}} b_{k}$, such that each factorization is unique.

Then, $p \cdot \prod_{h_{i} \in B_{h}} h_{i}=\prod_{a_{j} \in B_{a}} a_{j} \cdot \prod_{b_{k} \in B_{b}} b_{k}$.

By uniqueness, each irreducible on $[p] \cup B_{h}$ is an associate of an irreducible in $B_{a} \cup B_{b}$, so $[p] \cup B_{h}$ is a subset of $B_{a} \cup B_{b}$, up to association.

In particular, this means $p$ associates with one irreducible in the factorization of $a b$, which means that either $p \mid a$ or $p \mid b$. This shows every irreducible of $Q$ is prime. 
We will make use of the following condition, which we name ACCP. We will prove later that the presence of this condition logically implies that every element admits a factorization into irreducibles.

Lemma 2.11. Let $R$ be an integral domain. The following are equivalent:

1. Every ascending chain of principal ideals is eventually constant,

2. In $R$, the divisibility relation is well-founded; that is, there is no infinite descending chain $\left\langle c_{i}\right\rangle_{i \in \omega}$ such that $c_{i+1}$ properly divides $c_{i}$.

Proof. We make use of the following facts:

Claim 1 For $a, b \in R, a \mid b$ if and only if $\langle a\rangle \subseteq\langle b\rangle$.

This fact is straightforward: if $a \mid b$, then $a \cdot r=b$ for some $r \in R$, which is equivalent to $\langle a\rangle \subseteq\langle b\rangle$.

Claim $2 a$ properly divides $b$ if and only if $\langle a\rangle \subsetneq\langle b\rangle$.

Now, if $a \cdot r=b$ for non-unit $r$, then $b=a \cdot r \notin\langle a\rangle$ whereas $a \in\langle b\rangle$ and if $\langle a\rangle=\langle b\rangle$ then $a \cdot r_{1}=b$ and $b \cdot r_{2}=a$, which makes $r_{1}$ and $r_{2}$ inverses of each other.

$1 . \Rightarrow 2$.

Let $\left\langle p_{i}\right\rangle_{i \in \omega}$ be an infinite descending chain in divisibility. In light of the claims, this corresponds to an infinite ascending chain of principal ideals, contradicting 1 .

2. $\Rightarrow 1$.

Let $\left\langle I_{i}\right\rangle_{i \in \omega}$ be a non-constant ascending chain of principal ideals. From the claims, this corresponds to an infinite descending chain in divisibility, which proves divisibility is not well-founded, contradicting 2.

Proposition 2.12. Let $B$ be a multiset of primes and $C$ a multiset of irreducibles. Then $\prod_{p \in B} p \mid \prod_{q \in C} q$ if and only if $B$ is a subset of $C$ up to association. Furthermore, the division is proper if and only if the subset inclusion is proper.

Proof. Suppose $\prod_{p_{i} \in B} p_{i} \mid \prod_{q_{j} \in C} q_{j}$. Since every $p_{i}$ is prime, we have by induction for all $p_{i}$ that $p_{i} \mid q_{j}$ for some $q_{j} \in C$. Define $f: B \rightarrow C$ by $f\left(p_{i}\right)=q_{j}$, where $j=\min \left\{j\left|p_{i}\right| q_{j}\right.$ and $\left.q_{j} \notin f\left[\left\{p_{1}, p_{2}, \cdots p_{i-1}\right\}\right]\right\}$. Notice that the choice of $j$ ensures injectivity of $f$. By primeness, $p_{i} \sim f\left(p_{i}\right)$. We claim this choice is always possible, and we prove this by induction on $|B|$. If $|B|=1$ with 
$p \in B$, then $p \mid \prod_{q_{j} \in C} q_{j}$ and an induction on $|C|$ shows using primeness of $p$ that $p$ divides exactly one element of $C$, which shows our choice for mapping $p$ under $f$ is possible. Suppose $|B|=t+1, B=\left[p_{1}, p_{2} \cdots p_{t}, p_{t+1}\right]$ and each $p_{i}$ with $i \leq t$ is mapped to $q_{i}$ under $f$. We have $\prod_{i \leq t+1} p_{i}=\prod_{q_{k} \in C} q_{k}$, and we can use cancellation to obtain $p_{t+1}=\prod_{q_{k} \in C, k \geq t+1} q_{k}$, and an induction on $\left|C \backslash f\left(\left[p_{1}, p_{2} \cdots p_{t}\right]\right)\right|$ shows using primeness of $p_{t+1}$ that $p_{t+1}$ divides exactly one element of $C \backslash f\left(\left[p_{1}, p_{2} \cdots p_{t}\right]\right)$, hence an injective choice for $f\left(p_{t+1}\right)$ is possible.

Also, if the division was proper, this means that $f$ was not surjective, which implies $B$ is a proper subset of $C$ up to association. This proves the first direction.

The second direction is easier: if $B$ subset of $C$ up to association, there is injective $f: B \rightarrow C$ such that $f(p)=q$ if $p \sim q$. This means $\prod_{p_{i} \in B} p_{i}$ $\prod_{p_{i} \in B} f\left(p_{i}\right) \mid \prod_{q_{j} \in C} q_{j}$. Again, if $B$ proper subset of $C$, then $f$ is not surjective, which implies the division is proper.

The following is a somewhat technical consequence of a ring being an UFD. We will make use of it in Chapter 4

Proposition 2.13. If $Q$ is a UFD, $a$ is irreducible in $Q, b, c \in Q$ and $m$ the greatest power of $a$ dividing $b, n$ the greatest power of $a$ dividing $c$, and suppose $m>n$. Then $b \nmid c$.

Proof. Let $b=\prod_{p_{i} \in B} p_{i}$ and $c=\prod_{q_{j} \in C} q_{j}$ be prime decompositions of $b$ and $c$ in Q.

By Proposition 2.12, $\prod_{p_{i} \in B} p_{i} \mid \prod_{q_{j} \in C} q_{j}$ if and only if $B$ is a subset of $C$ up to association.

Suppose $b \mid c$, in the light of the claim this means $t \leq v$ and there is an injective map $f:\{1,2 \cdots t\} \rightarrow\{1,2 \cdots v\}$ such that each $p_{i}$ with $i \leq t$ associates with some $q_{f(j)}$ for $j \leq v$.

Note that $a$ is prime, so if $a^{m} \mid b$ then without loss of generality $a=u_{i} p_{i}$ for units $u_{i}$ and $i \leq m \leq t$ and similarly since $a^{n} \mid c a=v_{j} q_{j}$ for units $v_{j}$ and $j \leq n \leq v$. 
But then $a=u_{i} p_{i}=v_{i} q_{f(i)}$ for all $i \leq m$, and so $a^{m} \mid c$. Since $m>n$ and we assumed $n$ is the largest such that $a^{n} \mid c$, we have a contradiction.

Since an isomorphism is a "structure-preserving map", it makes sense for many properties to be preserved under an isomorphism.

Proposition 2.14. If $R, Q$ are rings such that $R \cong Q$ via isomorphism $\varphi$, then

1. $\varphi\left(0_{R}\right)=0_{Q}$,

2. $\varphi\left(1_{R}\right)=1_{Q}$,

3. if $u \in R^{\times}$then $\varphi(u) \in Q^{\times}$,

4. if $p$ is irreducible in $R$, then $\varphi(p)$ is irreducible in $Q$,

5. if $p$ is prime in $R$, then $\varphi(p)$ is prime in $Q$,

6. if $R$ is an integral domain, then $Q$ is an integral domain,

7. if $R$ is a UFD, then $Q$ is a UFD.

The proof is straightforward, so we omit it.

\subsection{Localization}

A multiplicative subset of a ring $R$ is a subset of $R$ that contains $1_{R}$ and is closed under multiplication, but it does not contain $0_{R}$.

Definition 2.15. If $R$ is a ring and $a_{1}, a_{2} \cdots a_{k} \in R, P$ is a multiplicative subset of $R$ generated by $a_{1}, a_{2} \cdots a_{k}$ if it is the collection of finite products of elements in $\left\{a_{1}, a_{2} \cdots a_{k}\right\}$.

We use $r / a$ in the ring $R$ to denote the element $r \cdot a^{-1}$, for a unit $a$.

The localization of a ring $R$ with respect to a multiplicative set $S$ is a generalization of the idea of fraction. Intuitively, it contains precisely those "fractions" of the form $r / s$ where $r \in R$ and $s \in S$. We make this notion precise with the following definition.

Definition 2.16. Let $R$ be an integral domain, and $I$ be a multiplicative subset of $R$. Define a relation $\sim$ on $R \times I$ by setting $\langle r, a\rangle \sim\left\langle r^{\prime}, a^{\prime}\right\rangle$ if $r \cdot a^{\prime}=r^{\prime} \cdot a$ in $R$. Remark 2.17 shows that $\sim$ is an equivalence relation. The localization of $R$ by $I$, written $I^{-1} R$, is defined as the collection of $\sim$-equivalence classes.

We define the operations on $I \times R$ : 
$\langle r, a\rangle+_{I \times R}\left\langle r^{\prime}, a^{\prime}\right\rangle=\left\langle r a^{\prime}+r^{\prime} a, a \cdot a^{\prime}\right\rangle$, and $\langle r, a\rangle \cdot{ }_{I \times R}\left\langle r^{\prime}, a^{\prime}\right\rangle=\left\langle r \cdot r^{\prime}, a \cdot a^{\prime}\right\rangle$.

Operations on $I^{-1} R$ are defined by taking the equivalence classes of the operands and the results. Remark 2.18 shows these operations are welldefined. The multiplicative subset of $R$ generated by $a$ is $\left\{a^{k} \mid k \in \omega\right\}$. If $I$ is generated by a single element $a$, we denote the localization of $R$ by $I$ by $R_{a}$.

Remark 2.17. The relation $\sim$ on $R \times I$ of Definition 2.16 is an equivalence relation.

Proof. Reflexivity and symmetry of $\sim$ are immediate. For transitivity, let $\langle r, a\rangle \sim\left\langle r^{\prime}, a^{\prime}\right\rangle$ and $\left\langle r^{\prime}, a^{\prime}\right\rangle \sim\left\langle r^{\prime \prime}, a^{\prime \prime}\right\rangle$. Then $r \cdot r^{\prime} \cdot a^{\prime \prime}=r \cdot{ }_{Q} r^{\prime \prime} \cdot a^{\prime}$, multiplying by $a$ gives $r \cdot r^{\prime} \cdot a \cdot a^{\prime \prime}=r \cdot a \cdot r^{\prime \prime} \cdot a^{\prime}$, which is equivalent to $\left(r \cdot a^{\prime \prime}\right) \cdot\left(r^{\prime} \cdot a\right)=\left(r^{\prime} \cdot a\right) \cdot\left(r^{\prime \prime} \cdot a\right)$ and by cancellation, $r \cdot a^{\prime \prime}=r^{\prime \prime} \cdot a$.

Remark 2.18. The operations on $I^{-1} R$ are well-defined.

Proof. Let $\langle r, a\rangle \sim\left\langle r^{\prime}, a^{\prime}\right\rangle$ and $\langle p, b\rangle \sim\left\langle p^{\prime}, b^{\prime}\right\rangle$.

We need to show that $\left\langle r^{\prime} b^{\prime}+p^{\prime} a^{\prime}, a^{\prime} b^{\prime}\right\rangle \sim\langle r b+p a, a b\rangle$ and $\left\langle r^{\prime} p^{\prime}, a^{\prime} b^{\prime}\right\rangle \sim$ $\langle r p, a b\rangle$.

Note that $r a^{\prime}=r^{\prime} \cdot a$ and $p b^{\prime}=p^{\prime} \cdot b$.

For addition, we have: $\left(r^{\prime} b^{\prime}+p^{\prime} a^{\prime}\right) \cdot a b=r^{\prime} b^{\prime} a b+p^{\prime} a^{\prime} a b=(r b+p a) \cdot a^{\prime} b^{\prime}$, as required.

For multiplication, we have: $r^{\prime} p^{\prime} \cdot a b=r p \cdot a^{\prime} b^{\prime}$, as required.

We show below that a localization is always a ring and its elements can be written in the fraction form.

Remark 2.19. If $R$ is an integral domain and $I$ is a multiplicative subset of $R$, then the localization of $R$ by $I, I^{-1} R$, is a ring.

Proof. We note that $[\langle 0,1\rangle]$ and $[\langle 1,1\rangle]$ are the identities under addition and multiplication.

We note that the additive inverse of $[\langle r, a\rangle]$ is $[\langle-r, a\rangle]$, since $[\langle r, a\rangle]+$ $[\langle-r, a\rangle]=[\langle 0, a\rangle] \sim[\langle 0,1\rangle]$

Let $[\langle r, a\rangle],\left[\left\langle r^{\prime}, a^{\prime}\right\rangle\right]$ and $\left[\left\langle r^{\prime \prime}, a^{\prime \prime}\right\rangle\right]$ be elements of $I^{-1} R$.

That the operations on $I^{-1} R$ are well-defined was shown on Remark 2.18 . 
Commutativity of addition carries from $R:[\langle r, a\rangle]+\left[\left\langle r^{\prime}, a^{\prime}\right\rangle\right]=\left[\left\langle r \cdot a^{\prime}+\right.\right.$ $\left.\left.r^{\prime} \cdot a, a \cdot a^{\prime}\right\rangle\right]=\left[\left\langle r^{\prime} \cdot a+r \cdot a^{\prime}, a^{\prime} \cdot a\right\rangle\right]=\left[\left\langle r^{\prime}, a^{\prime}\right\rangle\right]+[\langle r, a\rangle]$.

Commutativity of multiplication carries as well: $[\langle r, a\rangle] \cdot\left[\left\langle r^{\prime}, a^{\prime}\right\rangle\right]=[\langle r$. $\left.\left.r^{\prime}, a \cdot a^{\prime}\right\rangle\right]=\left[\left\langle r^{\prime} \cdot r, a^{\prime} \cdot a\right\rangle\right]=\left[\left\langle r^{\prime}, a^{\prime}\right\rangle\right] \cdot[\langle r, a\rangle]$.

Associativity of addition: $[\langle r, a\rangle]+\left(\left[\left\langle r^{\prime}, a^{\prime}\right\rangle\right]+\left[\left\langle r^{\prime \prime}, a^{\prime \prime}\right\rangle\right]\right)=([\langle r, a\rangle]+$ $\left.\left[\left\langle r^{\prime}, a^{\prime}\right\rangle\right]\right)+\left[\left\langle r^{\prime \prime}, a^{\prime \prime}\right\rangle\right]=\left[\left\langle r a^{\prime} a^{\prime \prime}+r^{\prime} a a^{\prime \prime}+r^{\prime \prime} a a^{\prime}, a a^{\prime} a^{\prime \prime}\right\rangle\right]$.

Associativity of multiplication: $[\langle r, a\rangle] \cdot\left(\left[\left\langle r^{\prime}, a^{\prime}\right\rangle\right] \cdot\left[\left\langle r^{\prime \prime}, a^{\prime \prime}\right\rangle\right]\right)=([\langle r, a\rangle] \cdot$ $\left.\left[\left\langle r^{\prime}, a^{\prime}\right\rangle\right]\right) \cdot\left[\left\langle r^{\prime \prime}, a^{\prime \prime}\right\rangle\right]=\left[\left\langle r r^{\prime} r^{\prime \prime}, a a^{\prime} a^{\prime \prime}\right\rangle\right]$.

Distributivity carries from $R:[\langle r, a\rangle]\left(\left[\left\langle r^{\prime}, a^{\prime}\right\rangle\right]+\left[\left\langle r^{\prime \prime}, a^{\prime \prime}\right\rangle\right]\right)=[\langle r, a\rangle]$. $\left[\left\langle r^{\prime}, a^{\prime}\right\rangle\right]+[\langle r, a\rangle] \cdot\left[\left\langle r^{\prime \prime}, a^{\prime \prime}\right\rangle\right]=\left[\left\langle r r^{\prime} a^{\prime \prime}+r r^{\prime \prime} a^{\prime}, a a^{\prime} a^{\prime \prime}\right\rangle\right]$.

Remark 2.20. A ring $R$ is embedded into $I^{-1} R$ by the map $\varphi$ defined by $r \mapsto$ $[\langle r, 1\rangle]$.

Proof. $\varphi$ is injective since $r \cdot 1=p \cdot 1$ implies $r=p$.

We show $\varphi$ is a homomorphism. Additivity: $\varphi(r+p)=[\langle r+p, 1\rangle]$ while $\varphi(r)+\varphi(p)=[\langle r, 1\rangle]+[\langle p, 1\rangle]$ which is the same as $[\langle r+p, 1\rangle$. Multiplicativity is analogous.

At times we will choose to regard $R$ as a subring of $I^{-1} R$ and identify $R$ with its image under this embedding.

Remark 2.21. The equivalence class of $\langle r, a\rangle$ in $I^{-1} R$ is equal to $\varphi(r) / \varphi(a)$, where $\varphi$ is the canonical embedding $\varphi: R \rightarrow I^{-1} R$.

Proof. We note that $\varphi(a)$ is a unit of $I^{-1} R$, since $\varphi(a)=[\langle a, 1\rangle]$ and $[\langle a, 1\rangle]$. $[\langle 1, a\rangle]=[\langle 1,1\rangle]$.

Also note that $\varphi(r) / \varphi(a)=\varphi(r) \cdot(\varphi(a))^{-1}=[\langle r, 1\rangle] \cdot[\langle 1, a\rangle]=[\langle r, a\rangle]$.

Due to Remarks 2.19 and 2.21, we can write $r / a$ for the element $[\langle r, a\rangle]$ of $I^{-1} R$, and note that this notation is well-defined.

If the underlying ring $R$ is an integral domain or a UFD, the localization of $R$ by one of its multiplicative subsets is an integral domain or a UFD.

Lemma 2.22. Let $R$ be an integral domain and I be a multiplicative subset of $R$. Then $I^{-1} R$, the localization of $R$ by $I$, is an integral domain. 
Proof. Suppose $p / a \cdot r / b=0$, for $p, r \in R, a, b \in I$. Note that $p / a=0$ if and only if $p=0$ and assume $p / a \neq 0$ and $r / b \neq 0$, so $p \neq 0, r \neq 0$. But $p / a \cdot r / b=p r / a b$, so $p r=0$, so $R$ cannot be an integral domain.

Lemma 2.23. Let $R$ be an integral domain and $I$ a multiplicative subset of $R$. If $p \in R$ is prime, then in $I^{-1} R p$ is a unit or prime.

Proof. Let $a \in R$ be prime and not a unit of $I^{-1} R$. Suppose $a \mid r^{-1} b \cdot q^{-1} c$ in $I^{-1} R$. So $d^{-1} e \cdot a=r^{-1} b \cdot q^{-1} c$ for some $d^{-1} e \in I^{-1} R$. Then $a \nmid d$ in $R$ since $a$ is not a unit of $I^{-1} R$. So eqr $a=d b c$. Since $a$ is prime and $a \nmid d$, then it must be the case that $a \mid b$ or $a \mid c$ in $R$. But then $a \mid r^{-1} b$ or $a \mid q^{-1} c$ in $I^{-1} R$, as required.

Proposition 2.24. Let $R$ be an integral domain. If every element of $R$ has a prime factorization, then $R$ is a UFD.

Proof. We need to prove $R$ is Atomic and U-UFD.

Atomicity follows immediately from the assumption and the fact that every prime is irreducible.

Let $a \in R$. We know $a$ has a prime decomposition $B$. Suppose $C$ is another irreducible factorization of $a$. By Proposition 2.12, we know that $B$ is a subset of $C$ up to association. But if $B \neq C$ up to association, then $\prod_{q \in B} q$ properly divides $\prod_{p \in C} p$, which is not the case since they are associates.

Lemma 2.25. Let $R$ be a UFD and let $I$ be a multiplicative subset of $R$. Then $I^{-1} R$, the localization of $R$ by $I$, is a UFD.

Proof. By Lemma 2.22, $I^{-1} R$ is an integral domain.

We note that prime elements in $I$ become units in $I^{-1} R$. This is immediate, since all elements of $I$ are invertible in $I^{-1} R$.

Take an arbitrary non-zero element $r^{-1} a$ of $I^{-1} R$. Let the prime factorization of $a$ in $R$ be $B$ with $a \sim \prod_{p_{i} \in B} p_{i}$. We want to show that it is an associate of a product of primes of $I^{-1} R$. But $r^{-1} a$ is an associate of $a$, units of $R$ are also units of $I^{-1} R$ and if an element is prime in $R$, by Lemma 2.23 it is either 
a unit or a prime in $I^{-1} R$. Let $B^{\prime}$ be the multiset of elements of $B$ that are units in $I^{-1} R$, then $B \backslash B^{\prime}$ is the required prime factorization of $r^{-1} a$. By Proposition 2.24, the localization $I^{-1} R$ is a UFD.

\subsection{Polynomial Rings}

Next, we give a formal treatment of the ring of polynomials $R\left[x_{1}, x_{2} \cdots x_{k}\right]$ associated with a ring $R$. Informally, this comprises of the collections of finite expressions of the form $\sum_{\iota} r_{\iota} \bar{x}^{\iota}$, where $\iota$ is a multi-index. Formally, polynomials can be thought of as finite (nested) sequences of elements of $R$.

Definition 2.26. Let $R$ be a ring. A polynomial in one variable $f$ over $R$ is an element of $R^{<\omega}$, i.e. a finite tuple of the form $\left\langle r_{0}, r_{1}, r_{2} \cdots r_{n}\right\rangle$, where $r_{i} \in R$ for $0 \leq i \leq n, n \in \omega$ and $r_{n} \neq 0$. We write as shorthand $f(x)=\sum_{i=0}^{n} r_{i} x^{i}$ for the polynomial, and $R[x]$ for the class of all such polynomials. The operations on $R[x]$ are defined naturally as:

$$
\begin{aligned}
& \left(\sum_{i=0}^{n} r_{i} x^{i}\right)+{ }_{R[x]}\left(\sum_{i=0}^{n} t_{i} x^{i}\right)=\sum_{i=0}^{n}\left(r_{i}+t_{i}\right) x^{i}, \text { and } \\
& \left(\sum_{i=0}^{n} r_{i} x^{i}\right) \cdot R[x]\left(\sum_{i=0}^{m} t_{i} x^{i}\right)=\sum_{k=0}^{m+n}\left(\sum_{i+j=k} r_{i} \cdot t_{j}\right) x^{k} .
\end{aligned}
$$

By the degree of $f$ we mean the largest $i \leq n$ such that $r_{i} \neq 0$, and write $\operatorname{deg}(f)=i$. If $a \in R$, then $f$ evaluates at $a$ as $f(a)=\sum_{i=0}^{n} r_{i} a^{i}$.

We extend this to finitely many variables, and let $R\left[x_{1}, x_{2} \cdots x_{k-1}, x_{k}\right]=$ $R\left[x_{1}, x_{2} \cdots x_{k-1}\right]\left[x_{k}\right]$

To define a shorthand notation, we write $\bar{x}=x_{1}, x_{2} \cdots x_{k}$ and if $\iota=$ $i_{1}, i_{2} \cdots i_{k}$, where $i_{1}, i_{2} \cdots i_{k} \in \omega$, define $\bar{x}^{\iota}=x_{1}^{i_{1}} x_{2}^{i_{2}} \cdots x_{k}^{i_{k}}$, and write $g(\bar{x})=$ $\sum_{\iota} q_{\iota} \bar{x}^{\iota}$, where only finitely many $q_{\iota}$ are different from zero.

By the degree of $g$ relative to $x_{j}$ we mean the largest $i_{j} \in \iota$ with $q_{\iota} \neq 0$ and write $\operatorname{deg}_{x_{j}}(g)=i_{j}$.

If $a_{1}, a_{2} \cdots a_{k} \in R$, write $\bar{a}=a_{1}, a_{2} \cdots a_{k}$, and then $g$ evaluates at $\left\langle a_{1}, a_{2} \cdots a_{k}\right\rangle$ as $g(\bar{a})=\sum_{\iota} q_{\iota} \bar{a}^{\iota}$.

Note that the zero polynomial is coded by the empty string. 
We note in the following remark that if $\bar{x}$ is a permutation of $\bar{x}$, then $R[\bar{x}]=R\left[\overline{x^{\prime}}\right]$.

Remark 2.27. Note that $R\left[x_{n_{1}}, x_{n_{2}} \cdots x_{n_{k}}\right]$ for distinct $n_{1}, n_{2} \cdots n_{k} \in \omega$ refer to the same polynomial ring, namely $R\left[x_{1}, x_{2} \cdots x_{k}\right]$, therefore the indeterminate variables are not to be distinguished by order. We will sometimes write $R\left[y, x_{1}, x_{2} \cdots x_{k}\right]$ and this is the same as $R\left[x_{1}, x_{2} \cdots x_{k}, x_{k+1}\right]$.

We give a proof that the collection of polynomials associated with a ring $R$ forms a ring and further below that if $R$ is an integral domain, then $R[\bar{x}]$ is an integral domain.

Remark 2.28. If $R$ is a ring, then $R\left[x_{1}, x_{2} \cdots x_{k}\right]$ is a ring and $R$ embeds into $R\left[x_{1}, x_{2} \cdots x_{k}\right]$.

As is the case with localization, note that technically $R$ is only embedded into $R\left[x_{1}, x_{2} \cdots x_{k}\right]$, however at times we choose to regard $R$ as a subring of $R\left[x_{1}, x_{2} \cdots x_{k}\right]$ and identify it with its image under the embedding.

Proof. Let $f, g, h \in R[x]$ with $f(x)=\sum_{i=0}^{n} p_{i} x^{i}, g(x)=\sum_{j=0}^{m} q_{j} x^{j}$ and $h(x)=$ $\sum_{l=0}^{v} r_{l} x^{l}$

Note that $\bar{f}(x)=\sum_{i=0}^{n}\left(-p_{i}\right) x^{i}$ is an additive inverse of $f(x)$. Commutativity of addition carries from $R: f(x)+g(x)=g(x)+f(x)=\sum_{i=0}^{\max (m, n)}\left(p_{i}+q_{i}\right) x^{i}$, where we can assume a padding of the polynomial of lesser degree, that is $p_{i}$ or $q_{i}$ are taken as zero if $n<i \leq m$ or $m<i \leq n$. Commutativity of multiplication carries as well: $f(x) \cdot g(x)=g(x) \cdot f(x)=\sum_{k=0}^{m+n}\left(\sum_{k=i+j} p_{i} q_{j}\right) x^{k}$. Associativity of addition: $f(x)+(g(x)+h(x))=(f(x)+g(x))+h(x)=\sum_{i=0}^{\max (n, m, v)}\left(p_{i}+\right.$ $\left.q_{i}+r_{i}\right) x^{i}$, where we can assume a similar padding. Associativity of multiplication: $f(x) \cdot(g(x) \cdot h(x))=(f(x) \cdot g(x)) \cdot h(x) \sum_{k=0}^{n+m+v}\left(\sum_{k=i+j+l} p_{i} q_{j} r_{l}\right) x^{k}$. Distributivity carries from $R: f(x) \cdot(g(x)+h(x)) \stackrel{k=0}{=} f(x) g(x)+f(x) h(x)$ $n+\max (m, v)$

$$
\sum_{k=0}\left(\sum_{k=i+j} p_{i}\left(q_{j}+r_{j}\right)\right) x^{k}
$$

$0_{R}$ and $1_{R}$ serve as additive and multiplicative inverses in $R[x]$. By iteration, $R\left[x_{1}, x_{2} \cdots x_{k}\right]$ is a ring. Since $R$ is embedded in $R\left[x_{1}, x_{2} \cdots x_{k}\right]$ by the map $q \mapsto f$ for $f(\bar{x})=q$, the second part of the remark holds. 
Lemma 2.29. Let $R$ be an integral domain. Then $R\left[x_{1}, x_{2} \cdots x_{k}\right]$ is an integral domain.

Proof. Suppose $\sum_{i=0}^{n}\left(a_{i} x^{i}\right) \cdot \sum_{j=0}^{m}\left(b_{j} x^{j}\right)=0_{R}$, for $a_{i} \in R, b_{j} \in R$ for $0 \leq i \leq n$ and $0 \leq j \leq m$. Assume $\sum_{i=0}^{n}\left(a_{i} x^{i}\right) \neq 0$ and $\sum_{j=0}^{m}\left(b_{j} x^{j}\right) \neq 0$, and this implies $a_{n} \neq 0$ and $b_{m} \neq 0$. But then $\sum_{k=0}^{n+m}\left(\sum_{k=i+j} a_{i} \cdot b_{j}\right) x^{k}=0$. In particular, this means $a_{n} b_{m}=0$, so $R$ cannot be an integral domain. This shows $R[x]$ must be an integral domain. By iteration, $R\left[x_{1}, x_{2} \cdots x_{k}\right]$, must be an integral domain.

The units of an integral domain $R$ and the units of the associated ring of polynomials $R\left[x_{1}, x_{2} \cdots x_{k}\right]$ coincide.

Proposition 2.30. If $R$ is an integral domain, then $\left(R\left[x_{1}, x_{2} \cdots x_{k}\right]\right)^{\times}=R^{\times}$.

Proof. By Remark 2.28, $1_{R}=1_{R[x]}$.

If $r \in R^{\times}$then there is $r^{-1} \in R$ such that $r \cdot r^{-1}=1_{R}$. By Remark 2.28, $R<R[x]$, so $r^{-1} \in R[x]$.

Now suppose $f, g \in R[x]$ such that $f \cdot g=1_{R}$. Then, $\operatorname{deg}(f g)=\operatorname{deg}(1)$, so $\operatorname{deg}(f)+\operatorname{deg}(g)=0$ which means both $f$ and $g$ are constant, so $f, g \in R^{\times}$.

By iteration, $\left(R\left[x_{1}, x_{2} \cdots x_{k}\right]\right)^{\times}=R^{\times}$.

Each element in the collection of indeterminates $\left\{x_{1}, x_{2} \cdots x_{k}\right\}$ of $R\left[x_{1}, x_{2} \cdots x_{k}\right]$ is irreducible.

Proposition 2.31. If $R$ is an integral domain, in $R\left[x_{1}, x_{2} \cdots x_{k}\right]$ each $x_{i}$ with $1 \leq i \leq k$ is irreducible.

Proof. Suppose $g(\bar{x}) \cdot h(\bar{x})=x_{i}$. Then $\operatorname{deg}_{x_{i}}(g h)=\operatorname{deg}_{x_{i}}\left(x_{i}\right)$, so $\operatorname{deg}_{x_{i}}(g)+$ $\operatorname{deg}_{x_{i}}(h)=1$. Note that $\operatorname{deg}_{x_{j}}(g h)=0$ for $j \neq i \operatorname{sodeg} \operatorname{deg}_{x_{j}}(g)=0$ and $\operatorname{deg}_{x_{j}}(h)=$ 0 .

Suppose without loss of generality that $\operatorname{deg}_{x_{i}}(g)=0$ and $\operatorname{deg}_{x_{i}}(h)=1$. Then $h=a x_{i}+b, g=c$ for $a, b, c \in R$. Then $g h=a c x_{i}+b c$ and so $c \neq 0$ so $b=0$ and $c \cdot a=1$ which means $c \in R^{\times}$so $g \in R\left[x_{1}, x_{2} \cdots x_{k}\right]^{\times}$. 
The next result is known as the Factor Theorem, and it states that in $R[x]$ the element $a \in R$ is a zero of $f(x)$ if and only if $x-a$ divides $f(x)$.

Proposition 2.32 (Factor Theorem). If $R$ is an integral domain, $f \in R[x]$ and $a \in R$ then $f(a)=0$ if and only if there exists $g \in R[x]$ with $\operatorname{deg}(g)<\operatorname{deg}(f)$ such that $f(x)=(x-a) g(x)$.

Proof. If $f(x)=(x-a) g(x)$ then $f(a)=(a-a) g(a)=0 \cdot g(a)=0$.

Conversely, suppose $f(a)=0$, and suppose $f(x)=\sum_{i=0}^{n} r_{i} x^{i}$ with $r_{n} \neq 0$, so $\operatorname{deg}(f)=n$. Then, $f(x)=f(x)-f(a)=\sum_{i=0}^{n} r_{i}\left(x^{i}-a^{i}\right)$, but for any $m$, $x^{m}-a^{m}=(x-a)\left(x^{m-1}+x^{m-2} a+\cdots+a^{m-1}\right)$. Then $f(x)=(x-a) g(x)$, where $g(x)=\sum_{i=1}^{n} r_{i}\left(x^{i-1}+x^{i-2} a+\cdots+a^{i-1}\right)$, so $\operatorname{deg}(g)=n-1$.

We use Proposition 2.32 to show that a polynomial of degree $k$ can have at most $k$ zeros.

Proposition 2.33. If $R$ is an integral domain, then if $f \in R[x]$ with $f \neq 0$ and $\operatorname{deg}(f)=k$, there are at most $k$ elements of $R \alpha_{i}$ for $i \leq k$ such that $f\left(\alpha_{i}\right)=0$.

Proof. Proceed by induction on $k$. If $k=0$, the polynomial is constant and we can see there are no roots of $f(x)$.

Assume that if $k<n$, all $f \in R[x]$ have at most $k$ roots.

Let $k=n$ for some $f \in R[x]$. Let $a \in R$ such that $f(a)=0$.

By Proposition 2.32, $f(x)=(x-a) g(x)$ for some $g \in R[x]$ with $\operatorname{deg}(g)<$ $\operatorname{deg}(f)=n$. By induction hypothesis, $g(x)$ has at most $n-1$ roots.

If $f(x)$ has no other roots, we are done. Otherwise, let $b \in R, b \neq a$ such that $f(b)=0$. So $f(b)=(b-a) g(b)=0$, and we can see that $g(b)=0$, i.e. $b$ is a root of $g(x)$. We know there are at most $n-1$ choices for such a $b$. Thus, $f(x)$ has at most $n-1+1=n$ roots.

In any ring of polynomials $R[x]$ that contains the field of rationals, for any polynomial $f(x)$, there exists a unit $\alpha$ that is not a zero of $f$. Intuitively, this is because $\mathbb{Q}$ contains infinitely many units, while $f(x)$ can have only finitely many zeroes. 
Proposition 2.34. If $R$ is an integral domain and $\mathbb{Q}<R$, for any $f, g \in R[x]$ there is $\alpha \in R^{\times}$such that $f(\alpha) \neq 0$ and $g(\alpha) \neq 0$.

Proof. Let $n=\operatorname{deg}(f)$ and $m=\operatorname{deg}(g)$. By Proposition 2.33, there are at most $m+n$ zeroes of $f$ and $g$. But $\mathbb{Q}$ is an infinite field and $\mathbb{Q} \subseteq R^{\times}$. So $R^{\times}$is infinite, while the set of roots of $f$ and $g$ is finite.

The next lemma is known as Gauss' Theorem. We will use it in a construction of Chapter 4.

Lemma 2.35 (Gauss' Theorem). Let $R$ be a UFD. Then $R[x]$ is a UFD.

\subsection{Independence and Generation}

In this section we are concerned with the algebraic independence and generation of certain subrings of a ring; both notions involve sets of elements of a ring $R$. Intuitively, a set $I \subset R$ is algebraically independent over some ring $Q<R$ if no finite sequence of ring operations involving elements of $Q$ and elements of $I$ evaluates to $0_{R}$. Similarly, the ring generated by $I$ over $Q$ comprises of the collection of elements we obtain through such sequences of operations. We make these concepts precise in what follows.

Definition 2.36. If $Q<P$ are rings and $a_{1}, a_{2}, \cdots, a_{k} \in P$, the ring $Q\left[a_{1}, a_{2}, \cdots a_{k}\right]=\left\{f\left(a_{1}, a_{2} \cdots a_{k}\right) \mid f \in Q\left[x_{1}, x_{2}, \cdots x_{k}\right]\right\}$ is the subring of $P$ generated by $Q \cup\left\{a_{1}, a_{2}, \cdots, a_{k}\right\}$.

The ring $Q\left[a_{1}, a_{2} \cdots a_{k}\right]$ is the smallest subring of $P$ under inclusion which contains $Q \cup\left\{a_{1}, a_{2}, \cdots, a_{k}\right\}$. It is important to note that this ring depends on both $P$ and $Q$.

A generated ring will always be a subring of the ring in which we generate it.

Remark 2.37. If $Q<P$ and $a_{1}, a_{2} \cdots a_{k} \in P$, then $Q \leq Q\left[a_{1}, a_{2}, \cdots a_{k}\right] \leq P$.

We omit the proof of this remark. 
The subring of $P$ generated over $Q$ by a multiplicative inverse of an element $b$ of $Q$ can be viewed as a collection of "fractions" over $Q$ with denominators powers of $b$. Hence, such a collection of fractions is isomorphic to the localization $Q_{b}$. This is the gist of the following two propositions.

Proposition 2.38. If $Q<P$ are rings, $c \in Q$ and $c$ is invertible in $P$, then $Q\left[c^{-1}\right]=\left\{b / c^{k} \mid b \in Q, k \in \omega\right\}$.

Proof. By Definition 2.36, $Q\left[c^{-1}\right]=\left\{f\left(c^{-1}\right) \mid f \in Q[x]\right\}$. But if $f(x)=\sum_{i=0}^{n} q_{i} x^{i}$, then $f\left(c^{-1}\right)=\sum_{i=0}^{n} q_{i} c^{-i}=c^{-n}\left(\sum_{i=0}^{n} q_{i} c^{n-i}\right)=c^{-n} \cdot b$ where $b \in Q$, therefore $Q\left[c^{-1}\right]=\left\{b / c^{k} \mid b \in Q, k \in \omega\right\}$.

For rings $R, T, S$ and embeddings $\psi_{1}: R \rightarrow T$ and $\psi_{2}: R \rightarrow S$, we say $T \cong S$ over $R$ with isomorphism $\varphi$, if $\varphi$ is an isomorphism between $T$ and $S$ such that $\varphi \circ \psi_{1}=\psi_{2}$. In particular, $R<T$ and $R<S$ is a special case of this definition, given by $\varphi_{\mid R}=i d_{R}$.

Proposition 2.39. Let $P$ be a ring, and let $b \in P$ be non-zero. Let $Q<P$ be a ring. The following are equivalent:

1. $Q_{b} \cong P$ over $Q$,

2. $b$ is a unit of $P$ and $P=Q\left[b^{-1}\right]$.

Proof. Recall $Q_{b}=Q \times I / \sim$ where $I$ is the multiplicative subset of $Q$ generated by $b$ and $\left\langle q, b^{k}\right\rangle \sim\left\langle q^{\prime}, b^{k^{\prime}}\right\rangle$ if $q \cdot b^{k^{\prime}}=q^{\prime} \cdot b^{k}$. The embedding of $Q$ into $Q_{b}$ is defined by $q \mapsto\langle q, 1\rangle$ and the operations on $Q_{b}$ are given by: $\left\langle q, b^{k}\right\rangle+{ }_{Q_{b}}\left\langle q^{\prime}, b^{k^{\prime}}\right\rangle=\left\langle q b^{k^{\prime}}+q^{\prime} b^{k}, b^{k} b^{k^{\prime}}\right\rangle$ and $\left\langle q, b^{k}\right\rangle \cdot{ }_{Q_{b}}\left\langle q^{\prime}, b^{k^{\prime}}\right\rangle=\left\langle q q^{\prime}, b^{k} b^{k^{\prime}}\right\rangle$.

This is an instance in which we identify $Q$ with its image under the canonical embedding into $Q_{b}$.

1. $\Rightarrow 2$.

Let $\varphi$ be an isomorphism between $Q_{b}$ and $P$. Note that $\varphi_{\uparrow Q}=i d_{Q}$

Since $b$ is a unit of $Q_{b}$, let $c=\langle 1, b\rangle \in Q_{b}$ such that $b c=1$ in $Q_{b}$. Then, $b \varphi(c)=\varphi(b) \varphi(c)=\varphi(b c)=1$ in $P$, so $b$ is a unit of $P$.

We need to show $Q\left[b^{-1}\right] \subseteq P$ and $P \subseteq Q\left[b^{-1}\right]$. The first inclusion is by definition.

Let $p \in P$, we have that $\varphi^{-1}(p) \in Q_{b}$ and so it is of the form $\left[\left\langle c, b^{k}\right\rangle\right]$, so $b^{k} \varphi^{-1}(p)=c \in Q$. Since $\varphi_{\Gamma Q}=i d_{Q}$, we obtain $b^{k} p \in Q$, which means $p \in Q\left[b^{-1}\right]$. 
2. $\Rightarrow 1$.

By Proposition 2.38, $Q\left[b^{-1}\right]=\left\{c / b^{k} \mid c \in Q, k \in \omega\right\}$.

Define a map $\varphi: P \rightarrow Q_{b}$ by $\varphi(p)=\left[\left\langle c, b^{k}\right\rangle\right]$ where $p=c / b^{k}$. Note that if $\left\langle c^{\prime}, b^{k^{\prime}}\right\rangle \in\left[\left\langle c, b^{k}\right\rangle\right]$, then $p=c^{\prime} / b^{k^{\prime}}$, since $p \cdot b^{k}=c$ and $c^{\prime} \cdot b^{k}=c \cdot b^{k^{\prime}}$ so we have $c^{\prime} \cdot b^{k}=p \cdot b^{k} \cdot b^{k^{\prime}}$ which implies $c^{\prime}=p \cdot b^{k^{\prime}}$ and so $p=c^{\prime} / b^{k^{\prime}}$.

Bijectivity follows by definition of $\varphi$, we check additivity and multiplicativity:

$\varphi\left(c / b^{k}+c^{\prime} / b^{k^{\prime}}\right)=\varphi\left(\left(c b^{k^{\prime}}+c^{\prime} b^{k}\right) / b^{k} b^{k^{\prime}}\right)=\left[\left\langle c, b^{k}\right\rangle+\left\langle c^{\prime}, b^{k^{\prime}}\right\rangle\right]=\left[\left\langle c b^{k^{\prime}}+\right.\right.$ $\left.c^{\prime} b^{k}, b^{k} b^{k^{\prime}}\right\rangle$, while $\varphi\left(c / b^{k}\right)+\varphi\left(c^{\prime} / b^{k^{\prime}}\right)=\left[\left\langle c, b^{k}\right\rangle\right]+\left[\left\langle c^{\prime}, b^{k^{\prime}}\right\rangle\right]=\left[\left\langle c b^{k^{\prime}}+c^{\prime} b^{k}, b^{k} b^{k^{\prime}}\right\rangle\right]$, and $\varphi\left(c / b^{k} \cdot c^{\prime} / b^{k^{\prime}}\right)=\varphi\left(c c^{\prime} / b^{k} b^{k^{\prime}}\right)=\left[\left\langle c c^{\prime}, b^{k} b^{k^{\prime}}\right\rangle\right]$, while $\varphi\left(c / b^{k}\right)+\varphi\left(c^{\prime} / b^{k^{\prime}}\right)=$ $\left[\left\langle c, b^{k}\right\rangle\right]+\left[\left\langle c^{\prime}, b^{k^{\prime}}\right\rangle\right]=\left[\left\langle c c^{\prime}, b^{k} b^{k^{\prime}}\right\rangle\right]$.

This shows $\varphi$ is an isomorphism. It is an isomorphism over $Q$ since elements of $Q$ are mapped to elements of $Q_{b}$ by the canonical embedding $\psi_{1}$, to elements of $P$ by the inclusion map $\psi_{2}$, and $\varphi \circ \psi_{2}=\psi_{1}$, because $\varphi\left(\psi_{2}(q)\right)=\varphi(q)=[\langle q, 1\rangle]=\psi_{1}(q)$.

Proposition 2.40. If $R<Q$ are rings and $I, J \subset Q$, then $R[I][J]=R[I \cup J]$.

Proof. Let $I=\left\{i_{1}, i_{2} \cdots i_{k}\right\}$ and $J=\left\{j_{1}, j_{2} \cdots j_{t}\right\}$.

We need to show $R[I][J] \subseteq R[I \cup J]$ and $R[I \cup J] \subseteq R[I][J]$.

Let $r \in R[I][J]$. Then $r=f(\bar{j})$, with $f(\bar{x})=\sum_{\zeta} r_{\zeta} \bar{x}^{\zeta}$, where each $r_{\zeta} \in R[I]$, so $r_{\zeta}=g_{\zeta}(\bar{i})=\sum_{\iota} r_{\zeta, i^{i}}$, with $r_{\zeta, \iota} \in R$. Then we define $h \in R[I \cup J]$ as $h(\bar{x}, \bar{y})=$ $\sum_{\zeta} \sum_{\iota} r_{\zeta, \iota} \bar{x}^{\zeta} \bar{y}^{\iota}$, and we can see that $h(\bar{j}, \bar{i})=f(\bar{j})$. Hence, $r \in R[I \cup J]$.

For the other inclusion, suppose without loss of generality that $I$ and $J$ are disjoint and let $r \in R[I \cup J]$ and the previous argument reverses. So $r=$ $h(\bar{i}, \bar{j})=\sum_{\zeta} \sum_{\iota} r_{\zeta, \iota} \bar{i}^{\zeta} \bar{j}^{\iota}$, with $r_{\zeta, \iota} \in R$, we can define the family of polynomials $g_{\zeta} \in R[I]$ by $g_{\zeta}(\bar{y})=\sum_{\iota} r_{\zeta, \iota} \bar{y}^{\iota}$, and the polynomial $f(\bar{x})=\sum_{\zeta} g_{\zeta}(\bar{i}) \bar{x}^{\zeta}$, and we can see that $f(\bar{j}) \in R[I][J]$ and $f(\bar{j})=h(\bar{i}, \bar{j})=r$.

We now define algebraic independence.

Definition 2.41. Let $Q<P$ and $a_{1}, a_{2} \cdots a_{k} \in P$. The set $\left\{a_{1}, a_{2} \cdots a_{k}\right\}$ is algebraically independent or independent over $Q$ if for all non-zero $f \in Q\left[x_{1}, \ldots, x_{k}\right]$, 
$f\left(a_{1}, \ldots, a_{k}\right) \neq 0$.

If the set $\{a\}$ is algebraically independent over $Q$ we say the element $a$ is transcendental over $Q$.

Note that in $R\left[x_{1}, x_{2} \cdots x_{k}\right]$ the set $\left\{x_{1}, x_{2} \cdots x_{k}\right\}$ is independent over $R$. The proof of this is immediate.

If $\varphi: R \rightarrow S$ and $\psi: P \rightarrow Q$ are maps between rings with $R<P$ and $S<Q$, we say that $\psi$ extends $\varphi$ if $\psi_{\uparrow R}=\varphi$.

The following fact will be useful in many of our proofs.

Lemma 2.42. Suppose we have rings $R<P=R\left[a_{1}, a_{2} \cdots a_{k}\right]$ with $\left\{a_{1}, a_{2} \cdots a_{k}\right\}$ independent over $R$ and $\psi: R \rightarrow S$ a homomorphism. Let $R^{\prime}=\psi[R]<S$ and let $\psi^{*}: R\left[x_{1}, x_{2} \cdots x_{k}\right] \rightarrow R^{\prime}\left[x_{1}, x_{2} \cdots x_{k}\right]$ be defined by $\psi^{*}(f)=\sum_{\iota} \psi\left(q_{\iota}\right) \bar{x}^{\iota}$ for $f=\sum_{\iota} q_{\iota} \bar{x}^{\iota}$.

Let $b_{1}, b_{2} \cdots b_{k} \in S$. Then the map $f(\bar{a}) \mapsto \psi^{*}(f)(\bar{b})$ is the unique homomorphism $\bar{\psi}: P \rightarrow S$ such that $\bar{\psi}\left(a_{i}\right)=b_{i}$ for $i \leq k$ and $\bar{\psi}$ extends $\psi$.

Further, if $\psi$ is injective and $\bar{b}$ is independent over $R^{\prime}$, then $\bar{\psi}$ is injective.

Also, the range of $\bar{\psi}$ is $R^{\prime}\left[b_{1}, b_{2} \cdots b_{k}\right]$.

Proof. We claim $\bar{\psi}$ is well-defined: suppose $f(\bar{a})=g(\bar{a})$, then put $h(\bar{a})=$ $f(\bar{a})-g(\bar{a})$ and if $f \neq g$ then $h$ contradicts the independence of $\bar{a}$. So $f=g$, which means $g(\bar{a}) \mapsto \psi^{*}(f)(\bar{b})$ and the claim is proved.

We note that $\bar{\psi}$ is defined on all of $P$, by definition of $P$.

Let $f(\bar{x})=\sum_{\iota} q_{\iota} \bar{x}^{\iota}$ and $g(\bar{x})=\sum_{\iota} r_{\iota} \bar{x}^{\iota}$, for $q_{\iota}, r_{\iota} \in R$.

Check that $\bar{\psi}$ is a homomorphism. Note that $f(\bar{a})+g(\bar{a})=(f+g)(\bar{a})$ and $f(\bar{a}) \cdot g(\bar{a})=(f \cdot g)(\bar{a})$. Check additivity: $\bar{\psi}(f(\bar{a})+g(\bar{a}))=\bar{\psi}((f+$ $g)(\bar{a}))=\psi^{*}(f+g)(\bar{b})=\sum_{\iota} \psi\left(q_{\iota}+r_{\iota}\right) \bar{b}^{\iota}$ and since $\psi$ is a homomorphism, this is $\psi^{*}(f)(\bar{b})+\psi^{*}(g)(\bar{b})$. Multiplicativity is analogous.

Now $\bar{\psi}$ is injective if $\psi$ is injective and $\bar{b}$ is independent over $R^{\prime}$. To see this, let $f(\bar{a}) \mapsto \psi^{*}(f)(\bar{b})$ and $g(\bar{a}) \mapsto \psi^{*}(g)(\bar{b})$, and suppose $\psi^{*}(f)(\bar{b})=$ $\psi^{*}(g)(\bar{b})$. Since $\bar{b}$ is independent, we get $\psi^{*}(f)=\psi^{*}(g)$. By injectivity of $\psi$, this means $q_{\iota}=r_{\iota}$. Hence, $f=g$.

We claim $\bar{\psi}$ is onto $R^{\prime}[\bar{b}]$. To see this, let $q=\sum_{\iota} \psi\left(q_{\iota}\right) \bar{b}^{\iota}$ be a member of $R^{\prime}[\bar{b}]$ and we can see that $q=\bar{\psi}\left(\sum_{\iota} q_{\iota} \bar{a}^{\iota}\right)$. 
Finally, we prove uniqueness of $\bar{\psi}$. Let $\phi: P \rightarrow S$ be a homomorphism such that $\phi$ extends $\psi$ and $\phi\left(a_{i}\right)=b_{i}$. Let $c=f(\bar{a})$ be an element of $P$, written as above. Then $\phi(c)=\sum_{\iota} \phi\left(q_{\iota} \bar{a}^{\iota}\right)$, and since $\phi$ extends $\psi$ this is the same as $\phi(c)=\sum_{\iota} \psi\left(q_{\iota}\right) \phi\left(\bar{a}^{\iota}\right)$ and since $\phi\left(a_{i}\right)=b_{i}$, we finally get $\phi(c)=\sum_{\iota} \psi\left(q_{\iota}\right) \bar{b}^{\iota}=$ $\bar{\psi}(c)$.

If $Q$ is a UFD and subring of $R, \bar{b} \in R$ is independent over $Q$, then the subring of $R$ generated by $Q$ and $\bar{b}$ is a UFD.

Proposition 2.43. Let $Q$ be a UFD, $Q<R$ and $\left\{b_{1}, b_{2} \cdots b_{k}\right\} \subset R$ be algebraically independent over $Q$. Then $Q\left[b_{1}, b_{2} \cdots b_{k}\right]$ is a UFD.

Proof. By Lemma 2.35, $Q[x]$ is a UFD, and by iteration $Q\left[x_{1}, x_{2} \cdots x_{k}\right]$ is a UFD. By Lemma 2.42, we have an isomorphism $\varphi: Q\left[b_{1}, b_{2} \cdots b_{k}\right] \rightarrow$ $Q\left[x_{1}, x_{2} \cdots x_{k}\right]$ with $b_{i} \mapsto x_{i}$. By Proposition 2.14, $Q\left[b_{1}, b_{2} \cdots b_{k}\right]$ is a UFD.

Proposition 2.44. Suppose we have rings $Q<P$ with $b_{1}, b_{2} \cdots b_{k} \in P$. The following are equivalent:

1. $P \cong Q\left[x_{1}, x_{2} \cdots x_{k}\right]$ over $Q$, with $b_{i} \mapsto x_{i}$ for $1 \leq i \leq k$;

2. $\left\{b_{1}, b_{2} \cdots b_{k}\right\}$ is independent over $Q$ and $P=Q\left[b_{1}, b_{2} \cdots b_{k}\right]$.

Proof. 1. $\Rightarrow 2$.

First we argue that $\left\{b_{1}, b_{2} \cdots b_{k}\right\}$ is independent over $Q$. We note that $0_{P} \mapsto 0_{Q\left[x_{1}, x_{2} \cdots x_{k}\right]}$. Suppose for a contradiction we have some non-zero $f \in Q\left[x_{1}, x_{2} \cdots x_{k}\right]$ with $f(\bar{b})={ }_{P} 0$. By isomorphism, $f(\bar{x})={ }_{Q[\bar{x}]} 0$, contradicting injectivity. So $f(\bar{b}) \neq 0$ for any non-zero $f$.

Now apply Lemma 2.42 to $Q, P, \bar{x}$ and the embedding $\psi$ of $Q$ into $P$ which is the restriction of the isomorphism from $P$ to $Q\left[x_{1}, x_{2} \cdots x_{k}\right]$, to obtain an isomorphism $\bar{\psi}$ between $Q\left[b_{1}, b_{2} \cdots b_{k}\right]$ and $P$. Since $Q=\psi[Q]$, the range of $\bar{\psi}$ is $Q\left[b_{1}, b_{2} \cdots b_{k}\right]$, hence $P=Q\left[b_{1}, b_{2} \cdots b_{k}\right]$.

2. $\Rightarrow 1$.

Apply Lemma 2.42 to $Q, P, Q\left[b_{1}, b_{2} \cdots b_{k}\right],\left\{b_{1}, b_{2} \cdots b_{k}\right\}$ and the embedding $\psi: Q \rightarrow P$ to obtain a homomorphism $\bar{\psi}: P \rightarrow Q\left[x_{1}, x_{2} \cdots x_{k}\right]$ with 
$b_{i} \mapsto x_{i}$. Then, $\bar{\psi}$ is one to one and onto $Q\left[x_{1}, x_{2} \cdots x_{k}\right]$, so it is an isomorphism.

The following result states that if $a$ is transcendental over $R$ and $f(a)$ is irreducible, then $f(x)$ must be irreducible in $R[x]$.

Proposition 2.45. If $R<P$ are rings, $f \in R[x], a \in P$ transcendental over $R$ and $f(a)$ is irreducible in $R[a]$, then $f$ is irreducible in $R[x]$.

Proof. By Proposition 2.44, $R[a] \cong R[x]$ with $a \mapsto x$. This means $f(a) \mapsto f(x)$ under this isomorphism. By Proposition 2.14, $f$ is irreducible in $R[x]$.

We give an equivalent condition for the union of sets of elements to be independent over some ring.

Lemma 2.46. If $Q$ and $R$ are rings, with $Q<R$ and $J, K \subset R$ with $J \cap K=\emptyset$, then $J \cup K$ is independent over $Q$ if and only if $J$ is independent over $T$ and $K$ is independent over $Q[J]$.

Proof. Let $J=\left\{j_{1}, j_{2} \cdots j_{r}\right\}$ and $K=\left\{k_{1}, k_{2} \cdots k_{t}\right\}$.

Note that by Proposition 2.40, $Q\left[x_{1}, x_{2} \cdots x_{r}\right]\left[x_{r}, x_{r+1} \cdots x_{t}\right]=$ $Q\left[x_{1}, x_{2} \cdots x_{r+t}\right]$.

Suppose $J$ is independent over $Q$ and $K$ is independent over $Q[J]$. We show $J \cup K$ is independent over $Q$.

For suppose $f(\bar{j}, \bar{k})=0$, with non-zero $f \in Q\left[x_{1}, x_{2} \cdots x_{t+r}\right]$.

Write $f$ as $f(\bar{x}, \bar{y})=\sum_{\iota} f_{\iota}(\bar{x}) \bar{y}^{\iota}$, where $\bar{x}=x_{1}, x_{2} \cdots x_{r}, \bar{y}=y_{1}, y_{2} \cdots y_{t}$ and $f_{\iota} \in Q\left[x_{1}, x_{2} \cdots x_{r}\right]$.

Note that since $f \neq 0$, some $f_{\iota}(\bar{x})$ must be non-zero.

However, since $f(\bar{j}, \bar{k})=0$ and since $K$ is independent over $Q[J]$, it follows that each $f_{\iota}(\bar{j})$ must be zero, so there is a non-zero polynomial $f$ over $Q\left[x_{1}, x_{2} \cdots x_{r}\right]$ with $f(\bar{j})=0$. Since $J$ is independent over $Q$, this produces a contradiction.

Conversely, suppose $J \cup K$ is independent over $Q$. That $J$ is independent over $Q$ is almost immediate, since $J \subseteq J \cup K$ and from $f \in Q\left[x_{1}, x_{2} \cdots x_{r}\right]$ we can define $f^{*} \in Q\left[x_{1}, x_{2} \cdots x_{r+t}\right]$ with zero-coefficients for $x_{r+1}, x_{r+2} \cdots x_{r+t}$, so $f^{*}=f$. So if $J$ is not independent over $Q$, neither can $J \cup K$ be. 
We now show $K$ is independent over $Q[J]$. Let $g \in(Q[J])[\bar{y}]$, write $g$ as $g(\bar{y})=\sum_{\iota} g_{\iota}(\bar{j}) y^{\iota}$ where as before $\bar{x}=x_{1}, x_{2} \cdots x_{r}, \bar{y}=y_{1}, y_{2} \cdots y_{t}$, and each $g_{\iota} \in Q\left[x_{1}, x_{2} \cdots x_{r}\right]$. But then we can define $g^{*}$ over $Q$ as $g^{*}(\bar{x}, \bar{y})=\sum_{\iota} g_{\iota}(\bar{x}) y^{\iota}$, and we can see that if $g(\bar{k})=0$, then $g^{*}(\bar{j}, \bar{k})=0$, against the independence of $J \cup K$.

Lemma 2.47 and Remarks 2.48, 2.49 and 2.50 establish properties of elements in independent generating sets.

If an element is transcendental over a ring, then all its associates are also transcendental.

Lemma 2.47. If $Q<R$ are rings, $a \in R$ is transcendental over $Q$ and $a \cdot{ }_{R} u=b$ for some unit u of $R$, then $b$ is transcendental over $Q$.

Proof. Suppose $b$ is not transcendental over $Q$. So there is $f \in Q[x], f \neq 0$ and $f(x)=\sum_{i=0}^{n} q_{i} x^{i}$, such that $f(b)=0$.

Define $h \in Q[x]$ by $h(x)=\sum_{i=0}^{n} q_{i} u^{-i} x^{i}$, We claim $h \neq 0$. Otherwise, if $q_{i} u^{-i}=0$ for all $0 \leq i \leq n$, since we know $u \neq 0$ it must be that $q_{i}=0$ for all $i$, but this is impossible since $f \neq 0$.

Then, $h(a)=\sum_{i=0}^{n} q_{i} u^{i} a^{i}=q_{0}+q_{1} b+q_{2} b^{2}+\cdots+q_{n} b^{n}=f(b)=0$, therefore $a$ is not transcendental over $Q$.

No transcendental elements of a generating set can be units in the generated ring.

Remark 2.48. If $Q<R$ are rings and $Q\left[a_{1}, a_{2} \cdots a_{k}\right]<R$ where the set $\left\{a_{1}, a_{2} \cdots a_{k}\right\}$ is independent over $Q$, then no $a_{i}$ with $1 \leq i \leq k$ is invertible in $Q\left[a_{1}, a_{2} \cdots a_{k}\right]$.

Proof. Suppose $b \cdot a_{i}=1_{Q}$.

Now suppose $b \in Q\left[a_{1}, a_{2} \cdots a_{k}\right]$, so $g\left(a_{1}, a_{2} \cdots a_{k}\right)=b$, for some $g \in Q\left[x_{1}, x_{2} \cdots x_{k}\right]$. Define $f \in Q\left[x_{1}, x_{2} \cdots x_{k}\right]$ as $f\left(x_{1}, x_{2} \cdots x_{k}\right)=x_{i}$. $g\left(x_{1}, x_{2} \cdots x_{k}\right)-1$ and note that $f \neq 0$, and so we have $f\left(a_{1}, a_{2} \cdots a_{k}\right)=$ $a_{i} b-1=0$. In this case, $\left\{a_{1}, a_{2} \cdots a_{k}\right\}$ would not be independent over $Q$. 
No elements of an independent generating set associate or divide any elements of the base ring or other elements in the set.

Remark 2.49. If $Q<R$ are rings and $Q\left[a_{1}, a_{2} \cdots a_{k}\right]<R$ where the set $\left\{a_{1}, a_{2} \cdots a_{k}\right\}$ is independent over $Q$, then no $a_{i}$ with $1 \leq i \leq k$ divides or associates with any element of $Q \cup\left\{a_{1}, a_{2} \cdots a_{k}\right\} \backslash\left\{a_{i}\right\}$.

Proof. Suppose $b=a_{i} \cdot c$, where $c=g\left(a_{1}, a_{2} \cdots a_{k}\right)$ for $g \in Q\left[x_{1}, x_{2} \cdots x_{k}\right]$.

Now suppose $b \in\left\{a_{1}, a_{2} \cdots a_{k}\right\} \backslash\left\{a_{i}\right\}$, so $b=a_{j}$. Define $f \in$ $Q\left[x_{1}, x_{2} \cdots x_{k}\right]$ as $f\left(x_{1}, x_{2} \cdots x_{k}\right)=x_{i} g\left(x_{1}, x_{2} \cdots x_{k}\right)-x_{j}$ and note that $f \neq 0$ since $i \neq j$, and so we have $f\left(a_{1}, a_{2} \cdots a_{k}\right)=a_{i} c-b=0$.

If $b \in Q$, define $f \in Q\left[x_{1}, x_{2} \cdots x_{k}\right]$ similarly as $f\left(x_{1}, x_{2} \cdots x_{k}\right)=$ $x_{i} g\left(x_{1}, x_{2} \cdots x_{k}\right)-b$, which again gives $f\left(a_{1}, a_{2} \cdots a_{k}\right)=a_{i} c-b=0$.

In either case, $\left\{a_{1}, a_{2} \cdots a_{k}\right\}$ would not be independent over $Q$.

All elements of an independent generating set are irreducible in the generated ring.

Remark 2.50. If $Q<R$ are rings and $Q\left[a_{1}, a_{2} \cdots a_{k}\right]<R$ where the set $\left\{a_{1}, a_{2} \cdots a_{k}\right\}$ is independent over $Q$, then every $a_{i}$ with $1 \leq i \leq k$ is an irreducible of $Q\left[a_{1}, a_{2} \cdots a_{k}\right]$.

Proof. By Proposition $2.44 Q\left[a_{1}, a_{2} \cdots a_{k}\right] \cong Q\left[x_{1}, x_{2} \cdots x_{k}\right]$ over $Q$, with $a_{i} \mapsto x_{i}$ for $1 \leq i \leq k$. By Proposition 2.31, each such $x_{i}$ is irreducible in $Q\left[x_{1}, x_{2} \cdots x_{k}\right]$. By Proposition 2.14, each $a_{i}$ is irreducible in $Q\left[a_{1}, a_{2} \cdots a_{k}\right]$.

Given a polynomial $f$ over some ring $R$ containing "fractions", it will later be a useful trick to define a polynomial $g$ over a subring of $R$ that has no denominators. This is a convenient way of restricting the domain of the polynomials we deal with.

Lemma 2.51. If $Q$ and $R$ are rings such that $Q<R$ and $b \in Q$ is a unit of $R$, then for all $f \in Q\left[b^{-1}\right]\left[x_{1}, x_{2} \cdots x_{n}\right]$ there exists $N \in \omega$ such that $b^{N} f \in$ $Q\left[x_{1}, x_{2} \cdots x_{n}\right]$. 
Proof. Let $f \in Q\left[b^{-1}\right]\left[x_{1}, x_{2} \cdots x_{k}\right], f(\bar{x})=\sum_{\iota}\left(q_{\iota} \bar{x}^{\iota}\right.$ where each $q_{\iota} \in Q\left[b^{-1}\right]$.

For each $\iota$, let $I_{\iota}=\min \left\{k \in \omega \mid q_{\iota}=c \cdot b^{-k}, c \in Q\right\}$. Let $N=\max \left\{I_{\iota}\right\}$.

Then $b^{N} \cdot q_{\iota} \in Q$ for all $\iota$, so $b^{N} f \in Q\left[x_{1}, x_{2} \cdots x_{n}\right]$.

Lemma 2.52 gives the intersection of rings generated by different elements in terms of the intersection of the generating sets.

Lemma 2.52. If $P, Q, R, S$ are rings, with $P=R\left[b, b_{1} \cdots b_{k}\right], Q=R\left[b, b^{-1}\right]$ for $b, b_{1} \cdots b_{k} \in S$ and $b$ a unit of $S, P<S$ and $Q<S$ where $\left\{b, b_{1} \cdots b_{k}\right\}$ is independent over $R$, then $P \cap Q=R[b]$.

Proof. $R[b] \subseteq P \cap Q$ is immediate since $R[b] \subseteq R\left[b_{1} \cdots b_{k}\right][b]=R\left[b, b_{1} \cdots b_{k}\right]$ and $R[b] \subseteq R\left[b^{-1}\right][b]=R\left[\left[b, b^{-1}\right]\right]$.

To prove the other containment, suppose $c \in P \cap Q$, so $c \in P$ and $c \in Q$. Write $\bar{b}=b_{1}, b_{2} \cdots b_{k}$ and $\bar{y}=y_{1}, y_{2} \cdots y_{k}$. Since $c \in P, c=f(b, \bar{b})$, for some $f \in R[x, \bar{y}]$. Since $c \in Q$, using Proposition 2.38, $c=d / b^{k}$ where $d \in R[b]$ therefore $d=g(b)$, for some $g \in R[x]$.

Put $\bar{f}(x, \bar{y})=x^{k} f(x, \bar{y})$ and let $h(x, \bar{y})=\bar{f}(x, \bar{y})-g(x)$ and we can see that $h \in R[x, \bar{y}]$ and $h(b, \bar{b})=d-d=0$.

If $h(x, \bar{y}) \neq 0$ then $\{b, \bar{b}\}$ is not independent over $R$. So it must be that $h(x, \bar{y})=0$, which means $g(x)=\bar{f}(x, \bar{y})$ so $\bar{f} \in R[x]$ which means $f \in R[x]$ but this implies $c \in R[b]$, as required.

This concludes our exposition of the elements of Abstract Algebra we will be using in our proofs. The next Chapter is concerned with Computability and Reverse Mathematics, and also with a few results of Effective Algebra, building on the material presented here. 


\section{Chapter 3}

\section{Reverse Mathematics and Logic}

In this chapter we set out the logic machinery we will be employing in our proofs, while also proving relevant results concerning Effective Algebra.

We now present briefly the fundamental notions of Computability and the framework of Reverse Mathematics.

A set $S \subseteq \omega$ will often be equated with its characteristic function. If $\sigma=\left\langle s_{0}, s_{1} \cdots s_{k-1}\right\rangle$, we will write $\sigma(i)$ for the element $s_{i}$ and $|\sigma|$ for $k$. If $i \geq k$, we leave the function $\sigma(i)$ undefined. Recall that $S^{<\omega}$ denotes the collection of finite tuples on $S$.

We will make use of strings of natural numbers to index elements of some particular ring. We will work in Baire space ${ }^{\omega} \omega$ of sequences, or strings, of natural numbers. If $\sigma$ and $\tau$ are strings, we use $\sigma^{\wedge} \tau$ to denote appending $\tau$ to the end of $\sigma$, to form a third string. We denote by $\sigma\lceil n$ the initial segment of $\sigma$ of length $n$. A tree is a collection $T$ of strings $\sigma \in \omega^{<\omega}$ such that $T$ is closed under initial segments, i.e. if $\sigma \in T$ then any initial segment of $\sigma$ is in $T$. The empty string is represented by $\lambda$. The relation $\preceq$ on a collection of strings $T$ is defined by $\sigma \preceq \tau$ if $\sigma$ is an initial segment of $\tau$. We write $\sigma \prec \tau$ if $\sigma \preceq \tau$ and $\sigma \neq \tau$. The relations $\succeq$ and $\succ$ are the inverses of relations $\preceq$ and $\prec$. If $\sigma \preceq \tau$ or $\sigma \succeq \tau$, the strings are said to be comparable. Otherwise we call them incomparable, written $\sigma \mid \tau$. For two strings $\sigma$ and $\tau$, we denote by $\sigma \cap \tau$ their longest common initial segment. An infinite path on a tree $T \subseteq \omega^{<\omega}$ is a function $f: \omega \rightarrow \omega$ such that for all $n, f\lceil n$ is in $T$. We say $\tau$ is a child of $\sigma$ in $T$ if both strings are in $T$ and $\tau=\sigma^{\wedge} s$ for some $s \in \omega$. Write $\sigma=\tau^{-}$. Intuitively, a string $\tau$ will later be called terminal in $T$ 
if it is not the initial segment of an infinite path. This nomenclature is not standard, as a string is usually called terminal in the literature if it has no descendants. We will make this notion precise in Chapter 4, in such a way as to be definable within a certain axiomatic framework. A tree $T$ is finitely branching if any string in $T$ has finitely many children. $T$ is binary branching if elements of $T$ have at most two children.

\subsection{Notions from Computability Theory}

For the primitive notions of Computability we follow Soare in [27].

Computability Theory is the study of the effective content of Mathematics, developing the informal concept of an algorithm, or computation, into a more formal framework consisting of computable functions and the structures they act upon. These functions are rigorously defined on the set of natural numbers $\omega=\{0,1,2,3 \cdots\}$. More complicated structures can be embedded into $\omega$ using the technique of Gödel Numbering.

A partial function $\varphi: \omega^{k} \rightarrow \omega$ is a function $\varphi: D \rightarrow \omega$, where $D \subseteq \omega^{k}$. We denote the domain of $\varphi$ by $\operatorname{dom}_{\varphi}$.

A Turing Machine is an abstract model of computation that formalizes the notion of algorithm. Turing Machines compute partial computable functions.

A set $A \subseteq \omega$ is c.e. if it is the empty set or the range of a total computable function. Hence, a set is c.e. if it can be listed (or enumerated) effectively. A set is computable if it and its complement are c.e.

Assuming these details, Turing Machines can be constructed to compute most of the common functions of ordinary Mathematics. We equate the informal concept of effective/computable with the formally defined notion of Turing Computable. This idea is a version of the well-known Church's Thesis.

There exists a Universal Turing Machine. This provides us with an enumeration of the partial computable functions $\varphi_{0}, \varphi_{1}, \varphi_{2} \cdots$ and an enumeration of the c.e. sets $W_{0}, W_{1}, W_{2} \cdots$, where $W_{i}=\operatorname{dom}_{\varphi_{i}}$.

The most natural c.e. set is $\emptyset^{\prime}=\left\{e \mid \varphi_{e}(e) \downarrow\right\}$, known as the Halting Problem. This set is not computable. We denote by $\emptyset_{s}^{\prime}$ the result of enumerating $\emptyset^{\prime}$ for $s$ steps of some enumeration. 
In general, theorems could claim the existence of (non-computable) objects given other (non-computable) objects. Such a theorem claiming the existence of a particular object (output) given the existence of some other object (input) will be said to hold uniformly if there exists an effective way to pass from indices for the inputs to indices for the outputs. If such a proposition claims that for every computable function $g$ and number $b$ there is a computable function $h$ and number $d$ such that some property holds, then to say that the proposition is uniform is to say that there is a computable function $f$ such that for all $a$ and $b$, if $\varphi_{a}$ is total then $f(a, b)=\langle c, d\rangle, \varphi_{c}$ is total and the pair $\left\langle\varphi_{c}, d\right\rangle$ satisfies the conclusion of the proposition given the input $\left\langle\varphi_{a}, b\right\rangle$.

We will often say that $A$ computes $B$ if given access to a finite fragment of the characteristic sequence of $A$, we can decide membership for $B$.

We code sequences by elements of $\omega$ in a way which makes the basic relations and operations on strings (such as $\sigma \preceq \tau$ and $\langle\sigma, \tau\rangle \mapsto \sigma \cap \tau)$ computable.

For two sets $A, B \subseteq \omega$, their join is defined as:

$$
A \oplus B=\{2 a \mid a \in A\} \cup\{2 b+1 \mid b \in B\} .
$$

The join $A \oplus B$ is the least upper bound of the Turing degrees of $A$ and $B$.

\subsection{Reverse Mathematics}

Reverse Mathematics is carried out using the language, $\mathcal{L}_{2}$, of Second Order Arithmetic, $\mathcal{Z}_{2}$. It is the study of theories weaker than $\mathcal{Z}_{2}$. $\mathcal{L}_{2}$ is a two sorted first order language, which has two types of variables: number variables, which are denoted by lower-case letters, and set variables, which are denoted by upper-case letters. $\mathcal{L}_{2}$ also has two types of quantifiers, $\exists x, \forall x$ and $\exists X, \forall X$.

The axioms for $\mathcal{Z}_{2}$ come in three categories: axioms specifying the properties of $+, \cdot, 0,1,<, \in$, to which we add the induction axiom for sets:

$$
((0 \in X \wedge \forall n(n \in X \rightarrow n+1 \in X)) \rightarrow \forall n(n \in X))
$$


and a simplified version of the comprehension scheme for forming sets:

$$
\exists X \forall n(n \in X \leftrightarrow \phi(n)) .
$$

The Arithmetical Hierarchy is ubiquitous in Mathematical Logic and we define it here, for formulas of $\mathcal{L}_{2}$.

Definition 3.1 (Arithmetical Hierarchy). A formula $\psi$ is $\Sigma_{0}^{0}$ and $\Pi_{0}^{0}$ if it is logically equivalent to a first order formula with only bounded quantifiers.

A formula is classified as $\Sigma_{n+1}^{0}$ (or $\Sigma_{n+1}$ ) if it is logically equivalent to a formula of the form:

$$
\exists n_{1} \exists n_{2} \cdots \exists n_{k} \psi
$$

where $\psi$ is a $\Pi_{n}^{0}$ formula.

A formula is classified as $\Pi_{n+1}^{0}\left(\right.$ or $\left.\Pi_{n+1}\right)$ if it is logically equivalent to a formula of the form:

$$
\forall n_{1} \forall n_{2} \cdots \forall n_{k} \psi
$$

where $\psi$ is a $\Sigma_{n}^{0}$ formula.

Note that a formula of $\mathcal{L}_{2}$ is said to be arithmetical if it contains no set quantifiers.

The base system will use a weak form of induction, restricted to $\Sigma_{1}^{0}$ formulas:

Definition 3.2 ( $\Sigma_{1}^{0}$-Induction). The $\Sigma_{1}^{0}$-Induction scheme is given by the following formula:

$$
(\phi(0) \wedge \forall n(\phi(n) \rightarrow \phi(n+1)) \rightarrow \forall n \phi(n)),
$$

where $\phi(n)$ is a $\Sigma_{1}^{0}$ formula that can contain set variables.

Various versions of the axiom for comprehension will also be used.

Definition 3.3 (Axiom Schema of Arithmetic Comprehension). The axiom schema of arithmetic comprehension is as follows:

$$
\forall w_{1}, \ldots, w_{n} \exists B \forall x\left(x \in B \Leftrightarrow \phi\left(x, w_{1}, \ldots, w_{n}\right)\right),
$$

where the formula $\phi$ is in the Arithmetical Hierarchy. This essentially means: 
"there exists a set $B$ whose members are precisely those objects that satisfy the predicate $\phi . "$

Note that $\phi$ cannot contain set quantifiers, however set parameters are allowed.

Definition 3.4 (Recursive Comprehension). Recursive comprehension is defined as follows:

$$
\forall x(\phi(x) \leftrightarrow \psi(x)) \rightarrow \exists X \forall x(x \in X \leftrightarrow \phi(x)),
$$

where $\phi$ is $\Sigma_{1}^{0}$ and $\psi$ is $\Pi_{1}^{0}$ and $X$ is not free on either $\psi$ or $\phi$.

A model for $\mathcal{L}_{2}$ is a first order structure

$$
\mathcal{U}=\left(A, S_{A},+_{A},{ }_{A}, 0_{A}, 1_{A},<_{A}, \in_{S_{A}}\right),
$$

where the number variables range over $A$ and the set variables range over $S_{A} \subseteq \mathcal{P}(A)$ and the function, relation and constant symbols are interpreted as indicated in the context.

$\mathcal{Z}_{2}$ consists of basic arithmetic axioms, the comprehension axiom for every formula $\varphi$, and a second-order induction axiom. This theory is sometimes called "full second order arithmetic" to distinguish it from its subsystems, defined below.

The intended model for $\mathcal{Z}_{2}$ is $(\omega, \mathcal{P}(\omega),+, \cdot, 0,1,<, \in)$, but we can also have non-standard models, if the number variables would range over a nonstandard set or the set variables would range over a set smaller than the full powerset of $A$.

An $\omega$-model $M$ is an $\mathcal{L}_{2}$-model for which the first order part is standard, i.e. $A=\omega$. So $M$ can be viewed as a collection of sets of natural numbers, representing the range of the set variables in $\mathcal{L}_{2}$.

\subsection{Subsystems of $\mathcal{Z}_{2}$}

The five subsystems of $\mathcal{Z}_{2}$ most frequently used in Reverse Mathematics are $R C A_{0}, W K L_{0}, A C A_{0}, A T R_{0}$ and $\Pi_{1}^{1} C A_{0}$. The subscript 0 refers to the usage of restricted induction. 
The first and weakest subsystem of $\mathcal{Z}_{2}, R C A_{0}$, is named because of the recursive comprehension axiom and it includes the arithmetic axioms, the recursive comprehension scheme and restricted induction. $R C A_{0}$ includes the semi-ring axioms while addition and multiplication satisfy their recursive definition. $\Sigma_{1}^{0}$ induction is allowed, while a classic result of [6] shows that $\Pi_{1}^{0}$ induction is also admissible. The unique smallest $\omega$-model for $R C A_{0}$ is the collection $R E C$ of computable/recursive sets. The $\omega$-models of $R C A_{0}$ are the collections of subsets of $\omega$ which are closed under join and relative computability.

This system corresponds roughly to constructive mathematics. If a statement is provable in $R C A_{0}$, its effective or computable version will always be true.

The second subsystem of $\mathcal{Z}_{2}$ is $W K L_{0}$ and is named after the Weak König's Lemma. This is the statement "every infinite binary branching tree has an infinite path". As the name suggests, $W K L_{0}$ is made up by adding Weak König's Lemma to the axioms of $R C A_{0}$. WKL $L_{0}$ is strictly stronger than $R C A_{0}$ - we can see this because the effective version of the Weak König's Lemma is not true. One consequence of equivalences to $W K L_{0}$ is that the effective versions of the results fail to hold.

The third subsystem is dubbed $A C A_{0}$, for Arithmetic Comprehension Axiom, which comprises of $R C A_{0}$ plus the $\Sigma_{1}^{0}$ comprehension. This system can prove the Full König's Lemma and can define the Turing Jump of any set. $A C A_{0}$ allows us to form the set of natural numbers satisfying an arbitrary arithmetical formula, no matter how complex. In this framework, it is easy to prove the existence of the integers, the rationals, the reals (as Cauchy sequences of rationals), the complex numbers (as pairs of reals) and also show some of their properties.

It is then provable that $R C A_{0}$ and the restriction of the comprehension scheme to arithmetical formulas, which yield the arithmetical induction scheme, is equivalent to $A C A_{0}$. The unique smallest $\omega$-model for $A C A_{0}$ is then the collection $A R I T H$ of arithmetical sets. Among the $\omega$-models of $R C A_{0}$, the $\omega$-models for $A C A_{0}$ are characterized by the property of closure under Turing Jump.

The system $A T R_{0}$, short for arithmetic transfinite recursion, comprises of $A C A_{0}$, plus axioms which allow for the arithmetic comprehension to be 
iterated transfinitely, along any well-order.

Given that a well-ordering is an irreflexive linear ordering of a subset of the natural numbers having no infinite descending sequences, the $A T R_{0}$ main axiom asserts that "if ' $<$ ' is a well-ordering, then for any arithmetical formula $\phi(j, Y)$ there exists a set $X$ such that for all $j$ and $n,(j, n) \in X$ if and only if $\phi(j,\{(i, m) \mid m<n \wedge(i, m) \in X\})^{\prime \prime}$.

It is a result of Friedman that $A T R_{0}$ is equivalent to the statement that any two well-orderings are comparable. In particular, it is also equivalent to the assertion that the Turing Jump can be iterated along any countable well ordering starting with any set.

The final and strongest system that is usually considered in Reverse Mathematics is $\Pi_{1}^{1} C A_{0}$, which is strong enough to develop the basic structure theory of countable abelian groups. It consists of the system $A T R_{0}$ plus the axiom of $\Pi_{1}^{1}$ comprehension, which states that $\{x \in \omega \mid \phi(x)\}$ exists for any $\Pi_{1}^{1}$ formula $\phi(x)$. This system can prove the existence of Kleene's $\mathcal{O}$, which cannot be shown in $A T R_{0}$.

This concludes our exposition of the subsystems of $\mathcal{Z}_{2}$. For completeness, we have introduced all of the big five subsystems, however we will only refer to $R C A_{0}$ and $A C A_{0}$ in our proofs, using the first as a base system and proving an equivalence with the latter.

\subsection{Computability and Algebra}

Computability Theory operates with computable structures, so we define the computable counterpart of a ring.

Definition 3.5. A computable ring is a computable subset $R \subseteq \omega$, equipped with two computable binary operations + and $\cdot$ on $R$, together with two elements $0,1 \in R$, such that $(R, 0,1,+, \cdot)$ is a ring.

The embedding of a ring into its associated ring of polynomials is computable with computable image. Furthermore, if the ring is computable, we can effectively compute an index for this embedding.

We state this in the following proposition concerning computability, noting that further details regarding numberings can be found in [26]. 
Proposition 3.6. If $R$ is a computable ring, then $R\left[x_{1}, x_{2} \cdots x_{k}\right]$ is computable and the embedding $\varphi: R \hookrightarrow R\left[x_{1}, x_{2} \cdots x_{k}\right]$ is computable with computable image.

This is an occasion in which we need to make a distinction between $R$ and its image under its embedding into $R[\bar{x}]$ and note that the map is not an identity.

Proof. $R[x]$ is defined as tuples over $R$; this can be the empty tuple or tuples in which the last element is non-zero; using a Gödel numbering these tuples can be coded by natural numbers. The operations on the resulting structure are computable, since the ring $R[x]$ is specified by effective rules for addition and multiplication, based on the operations of $R$. By iteration, $R\left[x_{1}, x_{2} \cdots x_{k}\right]$ is also computable.

The embedding $\varphi: R \hookrightarrow R[x]$ is then defined as $q_{0} \mapsto\left\langle q_{0}\right\rangle$. Since the tuple $\left\langle q_{0}\right\rangle$ is effectively recognizable by the condition $i<1$, the map $\varphi$ is computable with computable image.

By iteration, the embedding of $R$ into $R\left[x_{1}, x_{2} \cdots x_{k}\right]$ is computable with computable image.

We argue that Proposition 3.6 holds uniformly.

Remark 3.7. Proposition 3.6 is effective.

Proof. From indices for the computable ring $R$ we can pass to indices for the ring $R\left[x_{1}, x_{2} \cdots x_{k}\right]$. This is because $R\left[x_{1}, x_{2} \cdots x_{k}\right]$ is effectively defined using elements of $R$, while its operations are based on the operations of $R$.

Also, from indices for the rings $R$ and $R\left[x_{1}, x_{2} \cdots x_{k}\right]$, we can pass to a computable index for $\varphi$, and an index for $\varphi[R]$.

The following remark notes that the operation of evaluating a polynomial of $R\left[x_{1}, x_{2} \cdots x_{k}\right]$ within a computable ring $R$ is a computable operation.

Remark 3.8. If $R<Q$ are computable rings, then the function $\langle f, \bar{a}\rangle \mapsto f(\bar{a})$ from $R[\bar{x}] \times Q^{n} \rightarrow Q$ is computable. 
Proof. $f(\bar{a})$ is obtained effectively by uniformly replacing $\bar{a}$ for $\bar{x}$ in $f(\bar{x})$ and then carrying the multiplications and additions in $Q$. Since $Q$ is computable, the process of obtaining $f(\bar{a})$ must be effective.

Remark 3.9. Let $R$ be a computable integral domain and $I$ a multiplicative c.e. subset of $R$. Let $\varphi$ be the embedding of $R$ into $I^{-1} R$. Then, there is a computable ring $S$, a computable embedding $\psi: R \rightarrow S$, and an isomorphism $\theta: I^{-1} R \rightarrow S$, such that $\psi=\theta \circ \varphi$.

Proof. Recall the relation $\sim$ on $R \times I$ is defined by $\langle r, b\rangle \sim\left\langle r^{\prime}, b^{\prime}\right\rangle$ if $r \cdot b^{\prime}=r^{\prime} \cdot b$.

Let $b_{1}, b_{2} \cdots$ be an enumeration of $I$ where $b_{k}=b^{k}$.

Define a relation $E$ on $R \times \omega$ by $\langle a, k\rangle E\left\langle a^{\prime}, k^{\prime}\right\rangle$ if $\left\langle a, b_{k}\right\rangle \sim\left\langle a, b_{k^{\prime}}\right\rangle$. Then $\mathrm{E}$ is a computable relation: to see if a pair $\langle a, k\rangle$ is in the relation we see the enumeration of $I$ up to step $k$ and checking whether two pairs are $\sim$ equivalent can be done effectively, since $R$ is computable.

Consider the collection $(R \times \omega) / E$ and the map $\eta: R \times \omega \rightarrow R \times \omega$ that chooses a representative for each $E$-equivalence class and sends each element to its representative. From an enumeration $\left\langle a_{1}, k_{1}\right\rangle,\left\langle a_{2}, k_{2}\right\rangle \cdots$ of $R \times \omega$ we can effectively do this by letting $\eta\left(a_{s}, k_{s}\right)=\left\langle a_{v}, k_{v}\right\rangle$ where $v=\min \{t \leq$ $\left.s \mid\left\langle a_{s}, b_{k_{s}}\right\rangle \sim\left\langle a_{t}, b_{k_{t}}\right\rangle\right\}$. In virtue of this definition, $\eta$ is computable with a computable image.

We let $S=\eta[R \times \omega]$.

Define $\theta: I^{-1} R \rightarrow S$ by $\theta\left(\left[\left\langle r, b_{k}\right\rangle\right]\right)=\eta(r, k)$. Bijectivity of $\theta$ follows from the definition of $\eta$.

Define the operations on $S$ by: $a+{ }_{S} b=\theta\left(\theta^{-1}(a)+{ }_{I^{-1}} \theta^{-1}(b)\right)$ and $a \cdot{ }_{S} b=$ $\theta\left(\theta^{-1}(a) \cdot{ }_{I^{-1} R} \theta^{-1}(b)\right)$ It follows that $S$ is a ring since $I^{-1} R$ is a ring, and $\theta$ is an isomorphism.

We claim $S$ is computable. For let $a, c \in S$, we can effectively find $r, s \in R$ and $k, l \in \omega$ such that $a=\langle r, k\rangle$ and $c=\langle s, l\rangle$. Using a search, we can effectively find $m \in \omega$ such that $b_{m}=b_{k} \cdot{ }_{R} b_{l}$. Then $a \cdot{ }_{S} c=\eta\left(r \cdot_{R} s, m\right)$ and $a+{ }_{S} c=\eta\left(r \cdot{ }_{R} b_{l}+{ }_{R} s \cdot{ }_{R} b_{k}, m\right)$.

It is straightforward that $\psi=\theta \circ \varphi$, since $\psi(r)=\eta(r, k)$ such that $b_{k}=1_{R}$ and $\theta(\varphi(r))=\theta\left(\left[\left\langle r, 1_{R}\right\rangle\right]\right)=\eta(r, k)$. The fact that $\psi$ is an embedding follows from the fact that both $\varphi$ and $\theta$ are embeddings. 
Remark 3.10. Proposition 3.9 is effective.

Proof. From an index for $R$ as a computable ring, and a c.e. index for the set $I$, we can effectively compute an index for $S$ as a computable ring, a computable index for $\psi$ and a computable index for the set $\psi[R]$.

Recall that $R_{b}$ is the localization of $R$ by the multiplicative subset $I=$ $\left\{b^{k} \mid k \in \omega, b \in R, b \neq 0_{R}\right\}$.

Remark 3.11. Let $R$ be a computable integral domain, let $a \in R$ be nonzero, and suppose that the relation $\left\{\langle r, k\rangle \in R \times \omega\left|b^{k}\right| r\right\}$ is computable. Then there is a computable integral domain $S$ and a computable embedding $\psi: R \rightarrow S$ with computable image, and an isomorphism $\theta: R_{b} \rightarrow S$ such that $\psi=\theta \circ \varphi$, where $\varphi$ is the canonical embedding of $R$ into $R_{b}$.

Proof. The construction of $S$ is exactly as in the proof of Remark 3.9.

Under the assumption that the divisibility relation is computable, we have that the image of $\psi$ is computable, since $\langle r, k\rangle \in S$ is in the range of $\psi$ if and only if $\left.b^{k}\right|_{R} r$.

Remark 3.12. Remark 3.11 is uniform.

Proof. Just as in the proof of Remark 3.10, with the added assumption that we have a computable index for the relation $\left\{\langle r, k\rangle \in R \times \omega\left|b^{k}\right| r\right\}$. With this added index we obtain a computable index for the set $\psi[R]$.

The following proposition will allow us to prove Corollary 3.21. Again, we need it to hold uniformly.

Proposition 3.13. If $Q$ and $P$ are computable rings with $P=Q[a]$ for $a \in P$ transcendental over $Q$, there is a computable embedding $\varphi: P \hookrightarrow Q[y, z]$, such that:

1. $\varphi(a)=y \cdot z$,

2. $\varphi[P]$ is computable,

3. $\varphi \uparrow Q=i d_{Q}$. 
Proof. This is an instance in which we can assume $Q<Q[y, z]$ and the embedding of $Q$ into $Q[y, z]$ is $i d_{Q}$. Note that $i d_{Q}$ is a homomorphism.

Since $Q<P=Q[a]$ and $a$ is transcendental over $Q$, we can apply Lemma 2.42 to $Q, P$ and $i d_{Q}$ to obtain the unique homomorphism $\varphi: P \rightarrow Q[y, z]$ such that $f(a) \mapsto(f)(y z)$, that extends $i d_{Q}$ with $\varphi(a)=y z$. We claim $y z$ is transcendental over $Q$, otherwise we have non-zero $f \in Q[x]$ with $f(y z)=0$ from which we can construct non-zero $g \in Q[y, z]$ with $g(y, z)=f(y z)=0$, but this is impossible.

We claim $\varphi$ is the required embedding. By definition, $\varphi(a)=y \cdot z$.

We claim $\varphi$ is computable. $Q[x]$ is computable. Fix an element $p \in P$. From an enumeration $f_{1}, f_{2} \cdots$ of $Q[x]$, check if $f_{i}(a)=p$, and if so, map $p$ to $f_{i}(y \cdot z)$.

Note that the image of $\varphi$ is computable, since a polynomial $f \in Q[y, z]$ is in the image of $Q$ if and only if $f(y, z)=\sum_{i, j=0}^{i=n, j=m} q_{i, j} y^{i} z^{j}$ with $q_{i, j}=0$ when $i \neq j$. Also, $f \in \varphi[Q]$ if and only if $q_{i, j}=0$ if $i>0$ or $j>0$. These conditions are recognizable, given the description of the polynomial $f$.

Remark 3.14. Proposition 3.13 is effective.

Proof. From computable indices for $Q$ and $P$ and the element $a$ we can effectively compute indices for $\varphi$ and for the set $\varphi[P]$.

If a computable ring $Q$ is embedded into a computable ring $S$ and $S$ is infinitely bigger than $Q$ and given superset $R$ of $Q$ that is infinitely bigger than $Q$, we can uniformly define a computable structure on $R$ and a computable isomorphism between $R$ and $S$.

Proposition 3.15. Given computable ring $Q$, a computable embedding $\psi: Q \hookrightarrow S$ with $\psi[Q]$ computable, where $S$ is a computable ring and $|S \backslash \psi[Q]|=\omega$ and given computable set $R \supset Q$ with $|R \backslash Q|=\omega$, then there are functions $+_{R}: R^{2} \rightarrow R$ and $\cdot_{R}: R^{2} \rightarrow R$ such that $\left(R,+_{R},{ }_{R}, 0_{Q}, 1_{Q}\right)$ forms a computable ring with $Q<R$, and a computable isomorphism $\eta: S \rightarrow R$ such that $\eta \circ \psi=i d_{Q}$. 
Proof. We want to define $\eta$ as a computable bijection $\eta: S \rightarrow R$, such that $\eta \circ \psi=i d_{Q}$.

To see that such a function exists, let $s_{0}, s_{1}, s_{2} \cdots \in S$ be an effective enumeration of $S$ and $r_{0}, r_{1}, r_{2} \cdots$ be an effective enumeration of $R \backslash Q$. Define $\eta$ as follows

$$
\eta\left(s_{i}\right)= \begin{cases}\psi^{-1}\left(s_{i}\right), & \text { if } s_{i} \in \psi[Q] \\ r_{j} \text { such that } j=\min \left\{j \mid r_{j} \notin \eta\left[\left\{s_{0}, s_{1} \cdots s_{i-1}\right\}\right]\right\}, & \text { otherwise }\end{cases}
$$

Then $\eta_{\lceil\psi[Q]}$ is bijective due to the fact that $\psi$ is an embedding, and $\eta_{\lceil S \backslash \psi[Q]}$ is surjective because we are mapping $s_{i}$ to a minimum $r_{j}$ and injective because $r_{j}$ was not mapped before. In virtue of our definition, $\eta$ is also computable and $\eta \circ \psi=i d_{Q}$.

Define the computable ring structure on $R$ by $s_{1}+{ }_{R} s_{2}=\eta\left(\eta^{-1}\left(s_{1}\right)+{ }_{S}\right.$ $\left.\eta^{-1}\left(s_{2}\right)\right)$ and $s_{1} \cdot R s_{2}=\eta\left(\eta^{-1}\left(s_{1}\right) \cdot_{S} \eta^{-1}\left(s_{2}\right)\right)$.

Because they are compositions of computable operations, these functions are computable and $\eta$ becomes the required isomorphism.

Remark 3.16. Proposition 3.15 is effective.

Proof. Form computable indices for the rings $Q$ and $S$, and computable indices for the sets $R$ and $\psi[Q]$ we can effectively obtain the enumerations $s_{0}, s_{1} \cdots$ and $r_{0}, r_{1} \cdots$ and pass to a computable index for the ring $R$ and a computable index for the map $\eta$.

If $Q$ is a computable ring and there is a computable superset $S$ of $Q$ infinitely bigger than $Q$ and an element $a$ of $S$ that is not in $Q$, we can uniformly put a computable structure on $S$ such that the ring $S$ is generated by $Q$ and the element $a$.

Corollary 3.17. If $Q$ is a computable ring, $S$ is a computable superset of $Q$ with $|S \backslash Q|$ infinite, then there are functions $+_{S}: S^{2} \rightarrow S$ and $\cdot_{S}: S^{2} \rightarrow S$ such that $\left(S,+_{S}, \cdot_{S}, 0_{Q}, 1_{Q}\right)$ is a computable ring, $Q$ is a subring of $S$, and there is some $a \in S$ such that $S=Q[a]$ and $a$ is transcendental over $Q$. 
Proof. From Proposition 3.6 we know $Q[x]$ is computable, and we have a computable embedding with computable image $\varphi: Q \hookrightarrow Q[x]$.

Now, we get by Proposition 3.15 a computable structure $\left(S,+{ }_{S},{ }_{S}, 0_{Q}, 1_{Q}\right)$ and an isomorphism $\eta: Q[x] \rightarrow S$. Let $a=\eta(x)$, we know $a \mapsto x$, i.e. $a$ is transcendental over $Q$. By Proposition $2.44 a$ is transcendental over $Q$ and $S=Q[a]$.

Remark 3.18. Corollary 3.17 is effective.

Proof. Follows from Remark 3.7, Remark 3.16 and Proposition 3.6.

The next Corollary is analogous to Corollary 3.17, and it uniformly defines a computable structure on $S$ such that $S$ is isomorphic to $Q\left[c^{-1}\right]$, for a non-unit non-zero $c \in Q$.

Corollary 3.19. If $Q$ is a computable integral domain and $S$ is a computable set such that $Q \subset S$ and $|S \backslash Q|$ is infinite, and given some non-zero non-unit $c \in Q$ such that the relation $\left\{\langle r, k\rangle \in Q \times \omega\left|c^{k}\right| r\right\}$ is computable, then there are functions $+_{S}: S^{2} \rightarrow S$ and ${ }_{S}: S^{2} \rightarrow S$ such that $\left(S,+_{S},{ }_{S}, 0_{Q}, 1_{Q}\right)$ forms a computable integral domain, with $Q$ a subring of $S, c$ a unit of $S$, and $S=Q\left[c^{-1}\right]$.

Proof. Note that $Q_{c}=I^{-1} Q$, where $I=\left\{c^{k} \mid k \in \omega\right\}$, a c.e. set.

From Remark 3.9, we know there is a computable ring $P$, a computable embedding $\psi: Q \rightarrow P$ with computable image and an isomorphism $\theta: Q_{c} \rightarrow P$ which extends $\psi$. Note that we are considering $Q$ as a subring of $Q_{c}$.

From Proposition 3.15, we obtain a computable structure on $S$ and an isomorphism $\eta: P \rightarrow S$ such that $\eta \circ \psi=i d_{Q}$.

We need to show that $c$ is a unit of $S$ and $S=Q\left[c^{-1}\right]$. This follows by Proposition 2.39 because $\eta \circ \theta$ is an isomorphism between $Q_{c}$ and $S$, over $Q$.

Remark 3.20. Corollary 3.19 is effective.

Proof. Follows from Remark 3.16, Remark 3.10, Remark 3.18 and Proposition 3.6. 
In Chapter 4 we will need a ring homomorphism that maps a particular element of its domain to the product of two elements of its range. We will start with a given ring $Q$ and use this result for defining a ring $R$ containing $Q$ in which a particular element of $Q$ is the product of two elements of $R$. The following Corollary will allow that.

Corollary 3.21. If $Q$ and $P$ are computable rings such that $Q<P$ and $P=Q[a]$ with a transcendental over $Q$, and $S$ is a computable set such that $P \subset S$ and $S \backslash P$ is infinite, then there are functions $+_{S}, \cdot_{S}: S^{2} \rightarrow S$ such that $\left(S,+_{S}, \cdot_{S}, 0_{Q}, 1_{Q}\right)$ is a computable ring, $P$ is a subring of $S$ and elements $b, c \in S$ transcendental over $Q$, such that:

1. $Q[b, c]=S$, and

2. $b \cdot{ }_{S} c=a$.

Proof. From Proposition 3.13 we obtain a computable embedding $\varphi: P \rightarrow$ $Q[y, z]$ such that $\varphi(a)=y \cdot z$.

Apply Proposition 3.15 to $P, S$ and $Q[y, z]$ to put a computable structure on $S$ and obtain an isomorphism $\eta: Q[y, z] \rightarrow S$ such that $\eta \circ \varphi=i d_{P}$.

Put $b=\eta(y)$ and $c=\eta(z)$ to obtain $\left.b \cdot{ }_{S} c=\eta(y) \cdot{ }_{S} \eta(z)=\eta(y \cdot Q[y, z] z)\right)$ $=\eta(\varphi(a))=i d_{P}(a)=a$, and the following diagram:

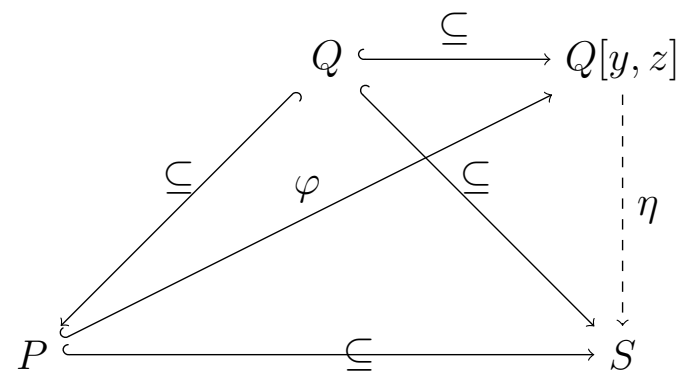

From Definition 2.41, $\{y, z\}$ is independent in $Q[y, z]$ over $Q$, so $\{b, c\}$ is independent in $S$ over $Q$. For if there is $f \in Q[y, z]$ such that $f(b, c)=0$, by isomorphism $f(y, z)=0$. This shows $S=Q[b, c]$ with $\{b, c\}$ independent over $Q$.

Remark 3.22. Corollary 3.21 is effective.

Proof. Follows from Remark 3.16, Remark 3.14 and Remark 3.7, and the fact that $b$ and $c$ can be found effectively, since the function $\eta$ from Proposition 3.15 can be found effectively. 
The following Proposition concerns computability of a generated ring, under the assumptions that the generating set is finite and the base ring is computable.

Proposition 3.23. If $Q<R$ are computable rings with $R=Q[J]$ for some finite $J \subseteq R$ independent over $Q$, and given $I \subset J$, then the ring $Q[I]$ is computable.

Proof. Let $I=\left\{a_{1}, a_{2} \cdots a_{k}\right\}$ and $J=\left\{a_{1}, a_{2} \cdots a_{r}\right\}$ with $r>k$. Then $Q[I]=$ $\left\{f\left(a_{1}, a_{2} \cdots a_{k}\right) \mid f \in Q\left[x_{1}, x_{2} \cdots x_{k}\right]\right\}$ and $R=Q[J]=\left\{g\left(a_{1}, a_{2} \cdots a_{r}\right) \mid g \in\right.$ $\left.Q\left[x_{1}, x_{2} \cdots x_{r}\right]\right\}$.

Let $\bar{x}=x_{1}, x_{2} \cdots x_{k}$ and $\bar{y}=a_{k}, a_{k+1} \cdots a_{r}$. Note that $Q[\bar{x}]$ is a computable subset of $Q[\bar{x}, \bar{y}]$.

By Proposition 2.44, fix a computable bijection $\psi: R \rightarrow Q[\bar{x}, \bar{y}]$. Then $a \in Q[I]$ if and only if $\psi(a) \in Q[\bar{x}]$, so $Q[I]$ is computable.

Remark 3.24. Proposition 3.23 is uniform.

Proof. From computable indices for the rings $Q$ and $J$ and for the sets $I$ and $J$, we can pass to computable indices for the ring $Q[I]$.

Finally, Lemma 3.25 gives a sufficient condition for the computability of a ring generated by two specific elements.

Lemma 3.25. If $P, Q, R$ are computable rings, $Q<R<P$ and $b$ invertible in $P$, with $R=Q\left[b, b_{1}, b_{2} \cdots b_{k}\right]$ where the presented variables are independent over $Q$, and $P=R\left[b^{-1}\right]$, then the ring $Q\left[b, b^{-1}\right]$ is computable.

Proof. By Proposition 3.23 applied to $R,\left\{b, b_{1}, b_{2} \cdots b_{k}\right\}$ and $\{b\}, Q[b]$ is computable.

Proposition 2.38 shows $P=\left\{c / b^{k} \mid c \in R, k \in \omega\right\}$.

Due to Remark 2.40, $Q\left[b, b^{-1}\right]=(Q[b])\left[b^{-1}\right]$. Since $Q[b] \subset P$, Proposition 2.38 also shows that $Q\left[b, b^{-1}\right]=\left\{c / b^{k} \mid c \in Q[b], k \in \omega\right\}$.

Given $d \in P$, we claim we can effectively find $c \in R$ and $k \in \omega$ such that $d=c / b^{k}$. Starting from enumerations $c_{1}, c_{2} \cdots \in R$ and $1, b, b^{2}, b^{3} \cdots$, we 
can consider all pairs $c, b^{k}$. We must eventually find such a pair and halt, because $d \in P$.

Now, if $d=c_{1} / b^{k_{1}}=c_{2} / b^{k_{2}}$ with $c_{1} \in Q[b]$ and $c_{2} \in R$, then $c_{2}=$ $c_{1} b^{k_{2}} / b^{k_{1}} \in Q\left[b, b^{-1}\right]$, so by Lemma $2.52 c_{2} \in Q[b]$.

Finally, we note that the condition $c \in Q[b]$ is decidable. This shows $Q\left[b, b^{-1}\right]$ is computable, for we can perform a search for $d=c / b^{k} \in P$ and decide whether $c \in Q[b]$.

Remark 3.26. Lemma 3.25 is uniform.

Proof. From computable indices for the rings $P, Q, R$ and the element $b$ we can pass to computable indices for the ring $Q\left[b, b^{-1}\right]$.

In the next Chapter we will define a particular sequence of rings. The first ring in the sequence will be isomorphic to the field of rationals $\mathbb{Q}$, hence we need a proposition that establishes an isomorphism between the rationals and an arbitrary countably infinite set.

Proposition 3.27. If $Q$ is a computable set with $|Q|=\omega$, there are computable functions $+_{Q}: Q^{2} \rightarrow Q$ and $\cdot_{Q}: Q^{2} \rightarrow Q$ and elements $0_{Q}, 1_{Q} \in Q$ such that $\left(Q,+_{Q}, \cdot_{Q}, 0_{Q}, 1_{Q}\right)$ forms a ring and $Q \cong \mathbb{Q}$.

Proof. Let $R$ be a computable copy of the integers, such that the set $Q$ contains $R$ and $|Q \backslash R|=\omega$. This can be achieved, for example, by coding the integers into the even numbers and letting $Q=\omega$.

Now let $I=R \backslash\left\{0_{R}\right\}$. Note that $I$ is a c.e. subset of $R$. By Remark 3.9, there is a computable ring $S$, a computable embedding $\psi: R \rightarrow S$ with computable image, and an isomorphism $\eta: I^{-1} R \rightarrow S$ that extends $\psi$.

Note that $I^{-1} R \cong \mathbb{Q}$, so $S \cong \mathbb{Q}$.

By Proposition 3.15, there is a computable structure on $Q$ such that $R<$ $Q$ and there exists isomorphism $\theta: S \rightarrow Q$ with $\theta \circ \psi=i d_{R}$.

This proves there exists an isomorphism between $Q$ and $\mathbb{Q}$.

This is the end of Chapter 3. The final Chapter is an exposition of our main results. 


\section{Chapter 4}

\section{Equivalent characterizations of UFDs}

In this chapter we present our results, which deal with the proof-theoretic strength of Theorems 1.2 and 1.3. We will work in the base system $R C A_{0}$.

\subsection{The theorems}

We will make use of the following two lemmas in the proof of Theorem 1.3.

Recall that $C \sim C^{\prime}$ means that there is a bijection $f: C \rightarrow C^{\prime}$ such that $p \sim f(p)$ for all $p \in C$. Let $\prod C=\prod_{c \in C} c$.

Lemma $4.1\left(R C A_{0}\right)$. Let $R$ be an integral domain. Let $C$ and $C^{\prime}$ be multisets of primes of $R$. If $C \sim C^{\prime}$ then $\prod C \sim \prod C^{\prime}$.

Proof. Let $C=\left[p_{1}, p_{2} \cdots p_{m}\right]$, so $C^{\prime}=\left[q_{1}, q_{2} \cdots q_{m}\right]$ and there is injective $f:\{1,2 \cdots m\} \rightarrow\{1,2 \cdots m\}$ such that $p_{k} \sim q_{f(k)}$ for all $k \in\{1,2 \cdots m\}$.

For $k \leq m$ let $C_{k}=\left[p_{1}, p_{2} \cdots p_{k}\right]$ and $C_{k}^{\prime}=\left[q_{f(1)}, q_{f(2)} \cdots q_{f(k)}\right]$.

We prove the $\Sigma_{1}$ statement $\prod C_{k} \sim \prod C_{k}^{\prime}$ using induction on $k$. Take the base case as $k=0$ and we observe that $C_{k}=C_{k}^{\prime}=\emptyset$, so $\prod C_{k}=1_{R}=$ $\prod C_{k}^{\prime}$. Assume the statement holds for $k<m$, so there is $u \in R^{\times}$such that $u \prod C_{k}=\prod C_{k}^{\prime}$. By assumption, there is $v \in R^{\times}$such that $v p_{k+1}=q_{f(k+1)}$. Then,

$$
(u v) \prod C_{k+1}=u \prod C_{k} \cdot v p_{k+1}=\prod C_{k}^{\prime} q_{f(k+1)}=\prod C_{k+1}^{\prime},
$$


as required.

Lemma $4.2\left(R C A_{0}\right)$. Let $R$ be an integral domain. Let $C$ be a multiset of elements of $R$, and let $p \in R$ be prime. If $p \mid \prod C$, then there is some $a \in C$ such that $p \mid a$.

Proof. Let $C=\left[a_{1}, a_{2} \cdots a_{m}\right]$.

We use induction on $k \leq m$ to show that there is some $j \leq k$ such that $p$ $a_{j}$, or $p \mid \prod\left[a_{k+1}, a_{k+2} \cdots a_{m}\right]$. For the base step $k=0$, this is the assumption $p \mid \prod C$. For the induction step, assume the statement holds for $k$. If there is some $j \leq k$ such that $p \mid a_{j}$, then certainly the statement holds for $k+1$. If not, since $p$ is prime and by induction

$$
p \mid \prod\left[a_{k+1}, a_{k+2} \cdots a_{m}\right]=a_{k+1} \prod\left[a_{k+2} \cdots a_{m}\right],
$$

either $p \mid a_{k+1}$ or $p \mid\left[a_{k+2} \cdots a_{m}\right]$, so the statement holds for $k+1$. So the statement holds for $m$, which means $p \mid a_{j}$ for some $j \leq m$ or $p \mid \prod \emptyset=1_{R}$, which is impossible since $p$ is a non-unit.

The first theorem we will be concerned with states that if every irreducible is prime in an integral domain, then every element has at most one factorization into irreducibles. This result is provable in $R C A_{0}$.

Theorem $1.3\left(R C A_{0}\right)$. If an integral domain is $A P$, then it is an U-UFD.

The proof is essentially a repeat of the proof of Proposition 2.12. We repeat it due to the fact that we are confined to the base system $R C A_{0}$.

Proof. Let $R$ be an AP domain. Let $B$ and $B^{\prime}$ be multisets of irreducibles of $R$, and suppose $\prod B \sim \prod B^{\prime}$. Write $B=\left[p_{1}, p_{2} \cdots p_{m}\right]$ and $B^{\prime}=\left[q_{1}, q_{2} \cdots q_{n}\right]$.

By $\Sigma_{1}$ induction on $k \leq n$ we prove the formula $\varphi(k)$ : "there is injective $f:\{1,2 \cdots k\} \rightarrow\{1,2 \cdots m\}$ such that for all $i \leq k, p_{i} \sim q_{f(i)}$. For the base case $k=0$, the empty function witnesses the formula holds. For the inductive case, suppose $f$ does the job on $\{1,2 \cdots k\}$. Let $C=\left[p_{1}, p_{2} \cdots p_{k}\right]$, $C^{\prime}=\left[q_{f(1)}, q_{f(2)} \cdots q_{f(k)}\right]$, and let $b=\prod B, b^{\prime}=\prod B^{\prime}, c=\prod C$ and $c^{\prime}=\prod C^{\prime}$. Let $d=\prod\left(B \backslash B^{\prime}\right)$ and $d^{\prime}=\prod\left(C \backslash C^{\prime}\right)$.

By Lemma 4.1, $c \sim c^{\prime}$. From the assumption $b \sim b^{\prime}$ we conclude $d \sim d^{\prime}$. In particular, $p_{k+1} \mid d^{\prime}$. By Lemma 4.2, there is $q \in C \backslash C^{\prime}$ such 
that $p \mid q$. By irreducibility of $q, p \sim q$. Let $i \leq m$ such that $q=q_{i}$, define $g:\{1,2 \cdots k+1\} \rightarrow\{1,2 \cdots m\}$ by letting $g(j)=f(j)$ for $j \leq k$ and $g(k+1)=i$.

The second theorem concerns the logical connection between wellfoundedness of divisibility and atomicity. The main contribution of our present work is to prove the equivalence of this result to $A C A_{0}$.

Theorem $1.2\left(A C A_{0}\right)$. If an integral domain satisfies the ACCP, then it is Atomic.

Proof. Let $R$ be a computable non-Atomic integral domain. There are two cases to consider.

Case 1: there is some $a \in R$ with no irreducible factor. Recursively define a sequence $\left\langle a_{i}\right\rangle_{i \in \omega}$ with $a_{0}=a$ and $a_{n+1}$ some proper factor of $a_{n}$. By induction, $a_{n}$ has no irreducible factor, so is reducible itself. $\emptyset^{\prime}$ can identify such $a_{n+1}$, so the sequence $\left\langle a_{i}\right\rangle_{i \in \omega}$ is computable from $\emptyset^{\prime}$. Since this is an infinite descending chain in divisibility, it is a counter-example to ACCP.

Case 2: every $b \in R$ has an irreducible factor, but some $a \in R$ is not the product of irreducible elements. Recursively define a sequence $\left\langle a_{i}\right\rangle_{i \in \omega}$ with $a_{0}=a$ and $a_{n+1}$ a proper factor of $a_{n}$ such that there is some irreducible $p_{n} \in R$ with $a_{n}=a_{n+1} \cdot R p_{n}$. By induction, $a_{n}$ is not the product of irreducible elements, and since $p_{n}$ is irreducible, this implies $a_{n+1}$ does not have an irreducible factorization. $\emptyset^{\prime \prime}$ can identify an irreducible factor of $a_{n}$ and so the sequence $\left\langle a_{i}\right\rangle_{i \in \omega}$ is computable from $\emptyset^{\prime \prime}$. This sequence is a counter-example to ACCP.

These theorems represent the first direction of the following equivalence, which is provable in $A C A_{0}$. The equivalence holds for an integral domain $R$ if and only if $R$ is a UFD.

Theorem $1.1\left(A C A_{0}\right)$. Let $R$ be an integral domain. The following are equivalent:

1. $R$ is an AP domain which satisfies the ACCP;

2. $R$ is an Atomic U-UFD.

Proof. 1. $\Rightarrow 2$. 
Theorems 1.3 and 1.2 prove this direction in $A C A_{0}$.

2. $\Rightarrow 1$.

Suppose every element has exactly one factorization into irreducibles in $R$. We need to prove $R$ satisfies the ACCP and it is an AP-domain.

Let $\left\langle c_{i}\right\rangle_{i \in \omega}$ be a descending chain in divisibility of $R$, that is $c_{i+1}$ divides $c_{i}$. Let $c_{i}=\prod_{p_{i, j} \in B_{i}} p_{i, j}$ be a factorization into irreducibles of $c_{i}$, where $B_{i}$ is a multiset of irreducibles. Note the use of $\emptyset^{\prime \prime}$ to construct this sequence of multisets, hence the proof uses $A C A_{0}$.

We claim $\left|B_{i+1}\right| \leq\left|B_{i}\right|$; for if $p_{1} p_{2} \cdots p_{k} \cdot \prod_{p_{i+1, j} \in B_{i+1}} p_{i+1, j}=\prod_{p_{i, j} \in B_{i}} p_{i, j}$, where each $p_{i}$ is an irreducible, by uniqueness of factorizations $\left|B_{i}\right|=k+\left|B_{i+1}\right|$. In turn, this means the sequence $\left|B_{i}\right|_{i \in \omega}$ is a non-increasing sequence of integers, so by Proposition 4.39, it stabilizes, which means there exists some $k$ such that $\left|B_{r}\right|=\left|B_{r+1}\right|$ for all $r \geq k$. By uniqueness of irreducible factorizations, this means $p_{r+1}$ does not divide $p_{r}$ properly.

The second part, that UFD implies AP, is given by Proposition 2.10.

For a complete discussion of factorization in integral domains, we refer the reader to [18].

Grams (1974, [10]) has given an example of an Atomic ring that does not satisfy the ACCP, hence providing a counter example to the converse of Theorem 1.2.

It was shown by Coykendall and Zafrullah (2004, [2]) that the converse of Theorem 1.3 does not hold. They have shown that there exist U-UFDs that are not APs.

We proceed now to showing that Theorem 1.2 implies $A C A_{0}$.

We start by constructing, in the next section, a tree $T$, encoding $\emptyset^{\prime}$ by its non-terminal elements.

We then proceed, in the following section, to defining an increasing sequence $\left\langle Q_{i}\right\rangle_{i \in \omega}$ of rings that encode precisely the elements of the tree, in such a way as to determine the divisibility relation in those rings by the descendant relation in $T$.

We prove the union of the rings in the sequence forms a computable integral domain $Q_{\omega}$, whose infinite descending chains in divisibility computes $\emptyset^{\prime}$. 
The conjuction of this result with Theorem 1.2 proves the existence of $\emptyset^{\prime}$ in a model of both, hence implying $A C A_{0}$.

The proofs that follow will be carried in $R C A_{0}$.

\subsection{A tree encoding $\emptyset^{\prime}$}

We define in stages trees $T_{n} \subset \omega^{<\omega}$ with $n \in \omega$. At each stage $k$, we have a corresponding string $\sigma_{k}$, which either gets extended to obtain $\sigma_{k+1}$ if it agrees with the configuration of $\emptyset_{k}^{\prime}$, or else $\sigma_{k+1}$ is assigned to the parent of $\sigma_{k}$, in a back-tracking step. In the first case, $\sigma_{k+1}$ is added to $T_{k}$ to obtain $T_{k+1}$, while in the second we simply assign $T_{k}$ to $T_{k+1}$.

This will provide an indexing for the elements of a ring we will construct later, which will help us determine the proof-theoretic strength of Theorem 1.2 .

The collection of strings is formally defined as follows:

Construction 4.3. Let $\sigma_{1}=\lambda$ and $T_{1}=\left\{\sigma_{1}\right\}$.

Step $k$, for $k \geq 1$ : If there exists $n \in \omega$ with $n<\left|\sigma_{k}\right|$ such that $\sigma_{k}(n)=0$ and $n \in \emptyset_{k+1}^{\prime}$, then $\sigma_{k+1}=\sigma_{k}^{-}$and $T_{k+1}=T_{k}$. Otherwise put $n=\left|\sigma_{k}\right|$ and let

$$
\sigma_{k+1}= \begin{cases}\sigma_{k}^{\curvearrowright} 0, & \text { if } n \notin \emptyset_{k+1}^{\prime} \\ \sigma_{k}^{\curvearrowright} s, & \text { where } n \in \emptyset_{k+1}^{\prime} \text { and } n \in \emptyset_{s}^{\prime} \backslash \emptyset_{s-1}^{\prime}\end{cases}
$$

with $T_{k+1}=T_{k} \cup\left\{\sigma_{k+1}\right\}$.

Finally, let $T=\bigcup_{n \in \omega} T_{n}$.

Note that $T$ is a computable tree in $\omega^{<\omega}$. Formally, we will not make use of this fact, as we will only need the sequence of strings $\left\langle\sigma_{i}\right\rangle_{i \in \omega}$. It is provable in $A C A_{0}$ that $T$ has a unique infinite path which computes $\emptyset^{\prime}$. Since we are working in $R C A_{0}$, we cannot use this fact, however it sheds some light on the reasons behind our definition of $T$ : we want an unbounded sequence in $T$ to compute the Halting Problem. Later, we will encode $T$ in a computable integral domain, and make use of an unbounded sequence in $T$ to show that some sequence of the ring computes the Halting Problem. 
Lemma 4.8 and Proposition 4.13 define what it means for a string $\sigma$ to be correct/non-terminal in $T$. As noted in Chapter 3, this terminology is non-standard.

For brevity, we say that a string $\sigma$ appears correct at stage $s$, if for all $n<|\sigma|$, $n \in \emptyset_{s}^{\prime}$ if and only if $\sigma(n)>0$. The next lemmas relate the notion of "appears correct" to Construction 4.3.

Lemma 4.4. Let $k \in \omega$, let $\sigma \in T_{k}$, and let $n<|\sigma|$. If $\sigma(n)>0$ then $n \in \emptyset_{k}^{\prime}$.

Proof. By induction on $k$. Suppose the statement is correct up to $k-1$. Let $\sigma \in T_{k}$. If $\sigma \in T_{k-1}$ then we are done since $\emptyset_{k-1}^{\prime} \subset \emptyset_{k-1}^{\prime}$. So suppose that $\sigma \in T_{k} \backslash T_{k-1}$, which implies that $\sigma=\sigma_{k}$, and $\sigma_{k}$ extends $\sigma_{k-1}$. Let $n<\left|\sigma_{k}\right|$. If $n<\left|\sigma_{k-1}\right|$ and $\sigma_{k}(n)>0$ then $\sigma_{k-1}(n)>0$ (as $\left.\sigma_{k-1} \prec \sigma_{k}\right)$ and so by induction $n \in \emptyset_{k-1}^{\prime} \subset \emptyset_{k}^{\prime}$. Otherwise $n=\left|\sigma_{k-1}\right|$ and by definition of $\sigma_{k}, \sigma_{k}(n)>0$ if and only if $n \in \emptyset_{k}^{\prime}$.

Lemma 4.5. Let $k \in \omega$. If $\sigma_{k}$ appears correct at stage $k+1$ then $\sigma_{k+1}$ is an extension of $\sigma_{k}$. Otherwise, $\sigma_{k+1}=\sigma_{k}^{-}$.

Proof. If $\sigma_{k}$ appears correct at stage $k+1$ then certainly the condition of the construction for letting $\sigma_{k+1}$ extend $\sigma_{k}$ holds. Suppose otherwise. Let $n<\left|\sigma_{k}\right|$ such that $\sigma_{k}(n) \neq 0$ if and only if $n \notin \emptyset_{k+1}^{\prime}$. By Lemma 4.4, $\sigma_{k}(n)>0$ is impossible, so $\sigma_{k}(n)=0$ and $n \in \emptyset_{k+1}^{\prime}$. Thus the condition in the construction for letting $\sigma_{k+1}=\sigma_{k}^{-}$holds.

Lemma 4.6. Suppose $\sigma_{k}$ is an extension of $\sigma_{k-1}$. Then $\sigma_{k}$ appears correct at stage $k$.

Proof. Let $n<\left|\sigma_{k}\right|$, we show that $n \in \emptyset_{k}^{\prime}$ if and only if $\sigma_{k}(n)>0$. There are two cases. First suppose that $n<\left|\sigma_{k-1}\right|$. By Lemma 4.5, $\sigma_{k-1}$ appears correct at stage $k$, or else $\sigma_{k}$ would not be an extension of $\sigma_{k-1}$. So $\sigma_{k}(n)=\sigma_{k-1}(n)>$ 0 if and only if $n \in \emptyset_{k}^{\prime}$ as required. Next take $n=\left|\sigma_{k}\right|$. Then $\sigma_{k}(n)>0$ if and only if $n \in \emptyset_{k}^{\prime}$ by the definition of $\sigma_{k}$.

Lemma 4.7. Let $\sigma \in T$ and let $k$ be the least such that $\sigma=\sigma_{k}$, so that $\sigma \in T_{k} \backslash T_{k-1}$. The collection of stages $s \geq k$ at which $\sigma$ appears correct forms an interval. 
Proof. It suffices to show that if $\sigma$ does not appear correct at some stage $s$, then it does not appear correct at stage $s+1$.

So suppose $\sigma$ does not appear correct at $s \geq k$. The assumption says that there is some $n<|\sigma|$ such that $\sigma(n)=0$ but $n \in \emptyset_{s}^{\prime}$. So either $\sigma(n)=0$ and $n \in \emptyset_{s}^{\prime}$, or $\sigma(n) \neq 0$ and $n \notin \emptyset_{s}^{\prime}$. However, the second is impossible by Lemma 4.4. So $\sigma(n)=0$ and $n \in \emptyset_{s}^{\prime}$, so $n \in \emptyset_{s+1}^{\prime}$ as well, and so witnesses that $\sigma$ does not appear correct at stage $s+1$.

Lemma 4.8. Let $\sigma \in T$ and let $k$ be the least such that $\sigma=\sigma_{k}$. For all $s \geq k$, if $\sigma$ appears correct at stage s, then $\sigma \preceq \sigma_{s}$.

Proof. We prove the lemma by induction on $k$. By Lemma 4.7, we can start with base case $k=s$, which is immediate since $\sigma_{k}=\sigma_{s}$.

Now assume $\sigma_{k} \preceq \sigma_{s}$ and $\sigma_{k}$ appears correct at step $s+1$, and show $\sigma_{k} \preceq \sigma_{s+1}$.

If $\sigma_{s+1}=\sigma_{s}^{\curvearrowright} s$, then $\sigma_{k} \preceq \sigma_{s} \prec \sigma_{s+1}$.

If $\sigma_{s+1}=\sigma_{s}^{-}$, there are two cases to consider: $\sigma_{s}=\sigma_{k}$ or $\sigma_{s} \succ \sigma_{k}$.

If $\sigma_{s}=\sigma_{k}$, since $\sigma_{s+1}=\sigma_{s}^{-}$, there is $j<\left|\sigma_{s}\right|$ such that $\sigma_{s}(j)=0$ and $j \in \emptyset_{s+1}^{\prime}$. This contradicts our assumption.

If $\sigma_{s} \succ \sigma_{k}$, then $\sigma_{s}=\sigma_{k}^{\wedge} s_{1}^{\imath} s_{2} \cdots s_{t}$ where $t>0$, so $\sigma_{s+1}=\sigma_{s}^{-}=$ $\widehat{\sigma_{k}} s_{1}^{\wedge} s_{2} \cdots s_{t-1}$, which shows $\sigma_{s+1} \succeq \sigma_{k}$.

Lemma 4.9. If $\sigma_{k}$ is extending $\sigma_{k-1}$, then $\sigma_{k} \notin T_{k-1}$.

Proof. let $k \in \omega$ and suppose, for a contradiction, that $\sigma_{k}$ is an extension of $\sigma_{k-1}$ but $\sigma_{k} \in T_{k-1}$. Let $m$ be the least stage such that $\sigma_{m}=\sigma_{k}$, so $m<k$ by the assumption for contradiction. By Lemma 4.6, $\sigma_{k}$ appears correct at both stages $m$ and $k$, so by Lemma 4.7, $\sigma_{k}$ appears correct at stage $k-1$. By Lemma 4.8, $\sigma_{k} \preceq \sigma_{k-1}$, contrary to the hypothesis.

We define a string $\sigma=\sigma_{k}$ to be correct if for all $n<\left|\sigma_{k}\right|, n \in \emptyset^{\prime}$ if and only if $\sigma_{k}(n) \neq 0$. Note that this is the same as $\sigma$ appearing correct at every stage $s \geq k$. We also use the notion of a string being non-terminal, which is equivalent to being correct. 
Proposition 4.10. If $\sigma$ is incorrect and $\tau \succeq \sigma$, then $\tau$ is incorrect.

Proof. Let $\sigma$ be incorrect in $T$ and let $\tau \succeq \sigma$. There exists $n<|\sigma|$ with $n \in \emptyset^{\prime}$ and $\sigma(n)=0$. The possibility $\sigma(n)>0$ and $n \notin \emptyset^{\prime}$ is excluded. Since $\tau \succeq \sigma$, the same property applies to $\tau$, which makes $\tau$ incorrect.

Lemma 4.11. For every $\tau \in T$, there is a string of greatest length $\sigma \preceq \tau$ which is correct.

Proof. We note that $\lambda$ is correct; this is immediate from definition.

Fix a string $\tau \in T$. If $\tau$ is correct, we are done. Otherwise, let $A=\{n \leq$ $|\tau| \mid \tau_{\lceil n}$ is not correct $\}$. The set $A$ is $\Sigma_{1}$ and non-empty, by $\Pi_{1}$ induction it has a least element.

Since $\lambda$ is correct, $n>0$. By Proposition 4.10, $\tau_{\lceil n-1}$ is the longest correct initial segment of $\tau$.

Lemma 4.12. Let $\rho \in T$ be incorrect, but suppose that $\tau=\rho^{-}$is correct. Then $\rho=\tau^{\frown} 0$, and there is some $s>0$ such that $\tau^{\wedge} s \in T$ is correct.

Proof. Let $n=|\tau|$. By definition, we know that if $\rho(n)>0$ then $n \in \emptyset^{\prime}$; but in that case, $\rho$ would be correct. Hence $\rho=\tau^{\wedge} 0$, and $n \in \emptyset^{\prime}$. Now, let $s$ be the greatest stage at which $\tau^{\wedge} 0$ appears correct, so $n \in \emptyset_{s+1}^{\prime} \backslash \emptyset_{s}^{\prime}$. By Lemma 4.7, $\rho=\tau^{\frown} 0$ does not appear correct at any stage $t>s$. So if $t \geq s$ and $\rho \preceq \sigma_{t}$, then $\sigma_{t}$ does not appear correct at stage $t$ and $\sigma_{t+1}=\sigma_{t}^{-}$. By induction we see that $\sigma_{s+1}=\sigma_{s}^{-}, \sigma_{s+2}=\sigma_{s+1}^{-} \cdots$ until $\sigma_{t}=\rho$, for $t=s+\left|\sigma_{s}\right|-|\rho|$, and $\sigma_{t+1}=\tau$. Since $\tau$ is correct, it appears correct at stage $t+2$ and so $\sigma_{t+2}=\tau^{\wedge} s$ and that string is also correct.

Proposition 4.13. Let $\sigma \in T$ and let $k$ be the least such that $\sigma=\sigma_{k}$. Then:

1. if $\sigma$ is correct, then for all $s \geq k, \sigma \preceq \sigma_{s}$,

2. if $\sigma$ is incorrect, then $\sigma \preceq \sigma_{s}$ for only finitely many $s$.

If 1 holds we say $\sigma$ is non-terminal in $T$, and if 2 holds we say it is terminal. 
Proof. Proof of 1. Follows from Proposition 4.8 and the fact that $\sigma$ appears correct at every stage $s \geq k$.

Proof of 2. Suppose that $\sigma$ is not correct. By Lemma 4.11, let $\tau$ be its longest initial segment that is correct and let $\rho=\sigma_{|| \tau \mid+1}$, so $\rho$ is not correct and $\tau=\rho^{-}$. By Lemma 4.12, $\rho=\tau^{\wedge} 0$ and there is some $s>0$ such that $\tau^{\wedge} s \in T$ is correct. By part 1, for all but finitely many stages $t$ we have that $\tau^{\wedge} s \preceq \sigma_{t}$. Since $\sigma \succ \tau \frown 0$ is incomparable with $\tau^{\wedge} s$, for every such stage $t$, $\sigma \npreceq \sigma_{t}$.

Proposition 4.13 provides us with the definition of "non-terminal", formulated in $R C A_{0}$, and shows this notion is equivalent to "correct".

Proposition 4.14. Any two non-terminal strings are comparable.

Proof. Let $\sigma, \tau$ be two non-terminal strings. Let $k$ be the least such that $\sigma \in T_{k}$ and $t$ be the least such that $\tau \in T_{t}$.

By Proposition 4.13, if $t \geq k$, then $\sigma \preceq \tau$, otherwise $\sigma \succ \tau$.

Proposition 4.15. Every correct string has a correct proper extension in $T$.

Proof. Let $\sigma \in T$ be correct, and let $k$ be the least such that $\sigma=\sigma_{k}$. If $\sigma_{k+1}$ is correct then we are done. Otherwise, Lemma 4.12 shows that $\sigma_{k+1}=\sigma^{\wedge} 0$ and that there is some $s>0$ such that $\sigma^{\wedge} s \in T$ and is correct.

For a sequence along a path in $T$, it must be that the elements of the sequence have a bigger size than their index in $T$.

Proposition 4.16. In an infinite sequence $\left(\tau_{i}\right)_{i \in \omega}$ consisting of elements of $T$ such that $\tau_{k} \prec \tau_{k+1}$, we have that $\left|\tau_{k}\right| \geq k$, for all $k \in \omega$.

Note that we are working in $R C A_{0}$ and we assume the sequence $\left(\tau_{i}\right)_{i \in \omega}$ exists, i.e. is an element of the second order part of the model. Hence the induction we will be performing in the proof is valid in $R C A_{0}$. 
Proof. We know $\left|\tau_{0}\right| \geq 0$ since lengths are non-negative. Assume $\left|\tau_{k}\right| \geq k$, for some $k \in \omega$.

Since $\left.\tau_{k} \prec \tau_{k+1}, \tau_{k+1}=\tau_{k}^{\imath} s_{1}^{\imath} s_{2} \ldots\right\urcorner s_{v}$, for $v \geq 1$. But then, $\left|\tau_{k+1}\right|=$ $\left|\tau_{k}\right|+v \geq\left|\tau_{k}\right|+1 \geq k+1$, so $\left|\tau_{k+1}\right| \geq k+1$.

We use Propositions 4.16 and 4.13 to show that an infinite sequence of descendants in $T$ computes $\emptyset^{\prime}$. Intuitively, this means an infinite path of $T$ computes the Halting Problem.

Lemma 4.17. Any infinite sequence $\left(\tau_{i}\right)_{i \in \omega}$ consisting of elements of $T$ such that $\tau_{k} \prec \tau_{k+1}$ computes $\emptyset^{\prime}$.

Proof. By Proposition 4.16, for all $k,\left|\tau_{k}\right| \geq k$.

We need to show that each $\tau_{k}$ is correct. This follows from Proposition 4.13 (2) and the fact that $\tau_{k}$ has infinitely many extensions on $T$, namely $\tau_{m}$ for $m>k$.

\subsection{Equivalence with $A C A_{0}$}

We will show there exist a computable non-Atomic integral domain in which every witness of the failure of ACCP computes $\emptyset^{\prime}$. Since $A C A_{0}$ is the weakest system that can prove the existence of the Turing Jump, this will give us our result.

The following proposition gives the sequence of underlying sets for the rings we define in Construction 4.19.

Proposition 4.18. There exists a uniformly computable sequence of sets $\left\langle Q_{n}\right\rangle_{n \in \omega}$ such that

1. $\left|Q_{0}\right|=\omega$,

2. $Q_{m} \subset Q_{m+1}$ and $\left|Q_{m+1} \backslash Q_{m}\right|=\omega$,

3. $\bigcup_{n \in \omega} Q_{n}=\omega$.

Proof. We will be implicitly using pairing functions, which means pairs $(m, x)$ are coded as elements of $\omega$. 
Define the sequence $\left(Q_{n}\right)_{n \in \omega}$ by $Q_{n}=\{(m, x) \mid x \in \omega, m \leq n\}$. The sequence is uniformly computable due to the fact that it can be effectively coded into $\omega$ using computable pairing functions.

Property 1 follows since $\left|Q_{0}\right|=|\{0\} \times \omega|=\omega$.

Since $Q_{m} \subset Q_{m+1}$ and $|\{m+1\} \times \omega|=\omega$, property 2 follows.

Property 3 follows because $\bigcup_{n \in \omega} Q_{n}=\omega \cdot \omega$.

Let $Q_{0}, Q_{1}, Q_{2} \cdots$ be the sequence obtained from Proposition 4.18. We are going to put computable ring structures on each of them in the following construction, which looks at the way Construction 4.3 is carried. Each $Q_{k+1}$ is a superset of $Q_{k}$ and it is generated as a ring over $Q_{k}$. We index its generators $a_{\sigma}, b_{\sigma}$, with elements of $T$. Each $Q_{k}$ will be a computable subring of $Q_{k+1}$.

To define $Q_{k+1}$ we look at how $T_{k+1}$ is obtained from $T_{k}$. If we back-track and make $\sigma_{k+1}=\sigma_{k}^{-}$, then we want $b_{\sigma_{k}}$ to be a unit in $Q_{k+1}$, otherwise we want $a_{\sigma_{k}}=a_{\sigma_{k+1}} b_{\sigma_{k+1}}$, in order to generate an infinite descending chain in divisibility in the rings.

More formally:

Construction 4.19. At step 0 , apply Proposition 3.27 to the set $Q_{0}$ to obtain $Q_{0} \cong \mathbb{Q}$, and let $R_{0}=Q_{0}$.

At step 1, apply Corollary 3.17 to $Q_{0}$ and $Q_{1}$ to obtain a computable structure on $Q_{1}$ and an element $a \in Q_{1}$ transcendental over $Q_{0}$, such that $Q_{1}=Q_{0}[a]$. Let $a_{\lambda}=a$, and we have $Q_{1}=Q_{0}\left[a_{\lambda}\right]=R_{0}\left[a_{\lambda}\right]$, such that $a_{\lambda}$ is transcendental over $R_{0}$. Let $R_{1}=R_{0}$.

Let $a=a_{\sigma_{k}}, b=b_{\sigma_{k}}$ and $\bar{b}=b_{\sigma_{k}\lceil n}$ for $n=1,2 \cdots\left|\sigma_{k}\right|$.

At step $k$, we have computable ring $Q_{k}=R_{k}[a, \bar{b}]$, where $R_{k}$ is a computable subring of $Q_{k}$ and the elements presented are algebraically independent over $R_{k}$.

At step $k+1$, if $T_{k+1}=T_{k}$ we want to make $b$ a unit. Since $Q_{k} \subset Q_{k+1}$, $\left|Q_{k+1} \backslash Q_{k}\right|$ is infinite, choose the element $b \in Q_{k}$ which is non-zero and non- 
unit by Remark 2.48, and since $Q_{k}$ is an integral domain by Corollary 4.22, apply Corollary 3.19 to put a computable structure on $Q_{k+1}$ that makes it a computable ring with $Q_{k}<Q_{k+1}, b$ a unit of $Q_{k+1}$ and $Q_{k+1}=Q_{k}\left[b^{-1}\right]$. Put $R_{k+1}=R_{k}\left[b, b^{-1}\right]$. Note that the conditions for applying Corollary 3.19 hold, in particular the relation $\left\{\langle q, m\rangle \in Q_{k} \times \omega\left|b^{m}\right| q\right\}$ is computable. To see this, note that $Q_{k} \cong R_{k}\left[y, x_{1}, x_{2} \cdots x_{\left|\sigma_{k}\right|}\right]$ over $R_{k}$ and this isomorphism is computable by Remark 3.8. Let $b=b_{\sigma_{k}}, q \in Q_{k}$ and $m \in \omega$, to tell whether $b^{m} \mid q$ find $a$ polynomial $f \in R_{k}[y, \bar{x}]$ such that $f(a, \bar{b})=q$ and check whether $x_{\left|\sigma_{k}\right|}^{m}$ divides $f$ in $R_{k}[y, \bar{x}]$; this is done by examining the coefficients of $f$ to see if the power of $x_{\left|\sigma_{k}\right|}$ is at least $m$ on any monomial with nonzero coefficient.

If $T_{k+1}=T_{k} \cup\left\{\sigma_{k+1}\right\}$, we have $Q_{k}=\left(R_{k}[\bar{b}]\right)[a]$ by Remark 2.40. Note that by Proposition $3.23, R_{k}[\bar{b}]$ is computable. and since $Q_{k+1} \supset Q_{k}$ we can apply Corollary 3.21 to $R_{k}[\bar{b}],\left(R_{k}[\bar{b}]\right)[a]$ and $Q_{k+1}$ to obtain the set $\{c, d\} \subset Q_{k+1}$ independent over $R_{k}[\bar{b}]$ and a computable structure on $Q_{k+1}$, such that $Q_{k+1}=\left(R_{k}[a, \bar{b}]\right)[c, d]$, i.e. $Q_{k+1}=Q_{k}[c, d]$ with $c \cdot Q_{Q_{k+1}} d=a$. We let $b_{\sigma_{k+1}}=c$ and $a_{\sigma_{k+1}}=d$. Put $R_{k+1}=R_{k}$.

Remark 4.20 shows the inductive definition preserves the inductive hypothesis.

Finally, let the union of each element of the sequence be a ring, $Q_{\omega}=\bigcup_{k \in \omega} Q_{k}$.

It is an essential ingredient of Construction 4.19 that the properties inferred in the inductive definition are preserved by induction. We prove this here.

Remark 4.20. In the inductive definition of Construction 4.19, $Q_{k+1}=$ $R_{k+1}\left[a_{\sigma_{k+1}}, b_{\sigma_{k+1} \mid n}\right]_{n=1,2 \cdots\left|\sigma_{k+1}\right|}$, with the elements presented being algebraically independent over $R_{k+1}$, and furthermore $R_{k+1}$ is a computable subring of $Q_{k+1}$.

Proof. We need to verify the induction hypothesis is preserved at step $k+1$. We need to show we have computable ring $Q_{k+1}=R_{k+1}\left[a_{\sigma_{k+1}}, b_{n}\right]_{n=1,2 \cdots\left|\sigma_{k+1}\right|}$, where $R_{k+1}$ is a computable subring of $Q_{k+1}$ and the elements presented are algebraically independent over $R_{k+1}$. 
If $T_{k+1}=T_{k}$ and we make $b_{\sigma_{k}}$ a unit, note that $\sigma_{k+1}=\sigma_{k}^{-}$from Construction 4.3. Let $a=a_{\sigma_{k}}, b=b_{\sigma_{k}}$ and $\bar{b}=b_{1}, b_{2} \cdots b_{\left|\sigma_{k}\right|-1}$.

We have $Q_{k+1}=Q_{k}\left[b^{-1}\right]$, so by inductive hypothesis $Q_{k+1}=$ $\left(R_{k}[a, b, \bar{b}]\left[b^{-1}\right]\right.$, by Remark $2.40 Q_{k+1}=\left(R_{k}\left[b, b^{-1}\right]\right)[a, \bar{b}]=R_{k+1}[a, \bar{b}]$. By definition, we have that $R_{k+1}<Q_{k+1}$. Since $\sigma_{k+1}=\sigma_{k}^{-}, a=a_{\sigma_{k+1}} \cdot b^{-1}$ and $b^{-1} \in R_{k+1}$, we have $Q_{k+1}=R_{k+1}\left[a_{\sigma_{k+1}}, b_{\sigma_{k+1} \mid n}\right]_{n=1,2 \cdots\left|\sigma_{k+1}\right|}$.

We need to show that $\left\{a_{\sigma_{k+1}}, \bar{b}\right\}$ is independent over $R_{k+1}$.

By Induction Hypothesis we know $\{a, b, \bar{b}\}$ is independent over $R_{k}$.

We claim it suffices to show $\{a, \bar{b}\}$ is independent over $R_{k+1}$. For if this is the case, then by Lemma $2.46\{a\}$ is independent over $R_{k+1}[\bar{b}]$ where $\bar{b}$ is independent over $R_{k+1}$, by Lemma $2.47\{a b\}$ is independent over $R_{k+1}[\bar{b}]$, and using Lemma 2.46 again and the fact that $a b=a_{\sigma_{k+1}}$, we obtain $\left\{a_{\sigma_{k+1}}, \bar{b}\right\}$ independent over $R_{k+1}$.

Now let $f \in R_{k+1}\left[x, y_{1}, y_{2} \cdots y_{\left|\sigma_{k}\right|-1}\right]$ such that $f \neq 0$. By Remark 2.40, $f \in\left(\left(R_{k}[b]\right)\left[b^{-1}\right]\right)\left[x, y_{1}, y_{2} \cdots y_{\left|\sigma_{k}\right|-1}\right]$. By Lemma 2.51, there is $N \in \omega$ such that $b^{N} f=g \in R_{k}[b][x, \bar{y}]$. By Lemma 2.46, $\{a, \bar{b}\}$ independent over $R_{k}[b]$ if and only if $\{a, b, \bar{b}\}$ independent over $R_{k}$. This last condition holds by Inductive Hypothesis, so $\{a, \bar{b}\}$ independent over $R_{k}[b]$, which means $g(a, \bar{b}) \neq 0$ which, since $b \neq 0$, means that $f(a, \bar{b}) \neq 0$, so the set $\{a, \bar{b}\}$ is independent over $R_{k+1}$.

Since $R_{k+1}=R_{k}\left[b, b^{-1}\right], R_{k}$ is computable and $b$ is transcendental over $R_{k}$, by Lemma $3.25 R_{k+1}$ is computable.

If $T_{k+1}=T_{k} \cup\left\{\sigma_{k+1}\right\}$, and we let $Q_{k+1}=Q_{k}\left[a_{\sigma_{k+1}}, b_{\sigma_{k+1}}\right]$ and $R_{k+1}=R_{k}$, then we have $\left.Q_{k+1}=\left(R_{k}\left[a_{\sigma_{k}}, b_{\sigma_{k} \mid n}\right]_{n=1,2 \cdots\left|\sigma_{k}\right|}\right]\right)\left[a_{\sigma_{k+1}}, b_{\sigma_{k+1}}\right]$ $=R_{k}\left[a_{\sigma_{k+1}}, a_{\sigma_{k}}, b_{\sigma_{k+1} \mid n}\right]_{n=1,2 \cdots\left|\sigma_{k+1}\right|}$, and since $a_{\sigma_{k}}=a_{\sigma_{k+1}} b_{\sigma_{k+1}}$ we have $a_{\sigma_{k}} \in R_{k}\left[a_{\sigma_{k+1}}, b_{\sigma_{k+1} \mid n}\right]_{n=1,2 \cdots\left|\sigma_{k+1}\right|}$ so $R_{k}\left[a_{\sigma_{k+1}}, a_{\sigma_{k}}, b_{\sigma_{k+1}\lceil n}\right]_{n=1,2 \cdots\left|\sigma_{k+1}\right|}=$ $R_{k}\left[a_{\sigma_{k+1}}, b_{\sigma_{k+1} \mid n}\right]_{n=1,2 \cdots\left|\sigma_{k+1}\right|}$, therefore $Q_{k+1}=R_{k}\left[a_{\sigma_{k+1}}, b_{\sigma_{k+1} \mid n}\right]_{n=1,2 \cdots\left|\sigma_{k+1}\right|}=$ $R_{k+1}\left[a_{\sigma_{k+1}}, b_{\sigma_{k+1} \backslash n}\right]_{n=1,2 \cdots\left|\sigma_{k+1}\right|}$.

The set $\left\{b_{\sigma_{k+1}\lceil n}|n=1,2 \cdots| \sigma_{k} \mid\right\}$ is independent over $R_{k+1}=R_{k}$, since it is a subset of variables at step $k$. The set $\left\{a_{\sigma_{k+1}}, b_{\sigma_{k+1}}\right\}$ is independent over $R_{k+1}\left[b_{\sigma_{k+1}}\lceil n]\right.$ in virtue of Corollary 3.21. By Lemma 2.46, $\left\{a_{\sigma_{k+1}}, b_{\sigma_{k+1}\lceil n}, \mid\right.$ $\left.n=1,2 \cdots\left|\sigma_{k+1}\right|\right\}$ is independent over $R_{k+1}$.

The rings defined in Construction 4.19 are UFDs. We will make use of 
this property extensively in later stages of our proof.

Proposition 4.21. Each ring in the sequence $\left\langle Q_{i}\right\rangle_{i \in \omega}$ is a UFD.

Proof. First we show that the rings in the sequence $\left\langle R_{i}\right\rangle_{i \in \omega}$ are UFDs. We use induction on $i$. $R_{0} \cong \mathbb{Q}, R_{0}$ is a UFD since it is isomorphic to a field, and a field is vacuously a UFD.

Assume $R_{k}$ is a UFD. We look at step $k+1$. If $R_{k+1}=R_{k}$ we are done. If $R_{k+1}=R_{k}\left[b_{\sigma}, b_{\sigma}^{-1}\right]=\left(R_{k}\left[b_{\sigma}\right]\right)\left[b_{\sigma}^{-1}\right]$, we use Proposition 2.43, since $b_{\sigma}$ is independent over $R_{k}$, to obtain that $R_{k}\left[b_{\sigma}\right]$ is a UFD. Then, by Lemma 2.25, the localization of $R_{k}\left[b_{\sigma}\right]$ on $b_{\sigma}, R_{k}\left[b_{\sigma}\right]_{b_{\sigma}}$ must be a UFD. By Proposition 2.39, $R_{k}\left[b_{\sigma}\right]_{b_{\sigma}} \cong\left(R_{k}\left[b_{\sigma}\right]\right)\left[b_{\sigma}^{-1}\right]$, so the latter must be a UFD.

So $R_{k+1}$ is a UFD.

Since at step $k$ we have computable ring $Q_{k}=R_{k}\left[a_{\sigma_{k}}, b_{\sigma_{k} \mid n}\right]_{n=1,2 \cdots\left|\sigma_{k}\right|}$, where $R_{k}$ is a UFD and the elements presented are algebraically independent over $R_{k}$, by Proposition 2.43 we deduce that $Q_{k}$ is a UFD.

This shows that each ring in the sequence must be a UFD.

As a corollary, each $Q_{i}$ must be an integral domain.

Corollary 4.22. Each ring in the sequence $\left\langle Q_{i}\right\rangle_{i \in \omega}$ is an integral domain.

Proof. By Proposition 4.21.

The obvious consequence is that $Q_{\omega}$ must be an integral domain.

Proposition 4.23. The ring $Q_{\omega}$ is an integral domain.

Proof. Suppose $a \cdot Q_{\omega} b=0_{Q_{\omega}}$ with $a \neq 0_{Q_{\omega}}$ and $b \neq 0_{Q_{\omega}}$. Then $a \cdot Q_{n} b=0_{Q_{\omega}}$ and neither elements are zero in $Q_{n}$, where $Q_{n}$ can be chosen as the smallest ring in the sequence containing all three elements. Such a ring must exist because all elements are added at finite stages, and they all contain the element $0_{Q_{\omega}}$.

So an element in the sequence $\left(Q_{i}\right)_{i \in \omega}$ is not an integral domain, which contradicts Corollary 4.22.

Since we are working in $R C A_{0}$, the structures we define need to be computable. This is the motivation behind the following proposition.

Proposition 4.24. The sequence of rings $\left(Q_{i}\right)_{i \in \omega}$ is uniformly computable. 
Proof. By Proposition 4.18 and Construction 4.19, the set $U=\{(q, n) \mid q \in$ $\left.Q_{n}\right\}$ is computable.

We need to show that the sets $P=\left\{(p, q, r, k) \mid p=q+_{Q_{k}} r\right\}$ and $T=$ $\left\{(p, q, r, k) \mid p=q \cdot Q_{k} r\right\}$ are computable. So let $q, r \in \bigcup_{i \in \omega} Q_{i}$. Since $U$ is computable, we can find the least $k_{1}, k_{2} \in \omega$ such that $q \in Q_{k_{1}}$ and $r \in Q_{k_{2}}$. Let $k=\max \left(k_{1}, k_{2}\right)$ and since $Q_{i}<Q_{i+1}$ for all $i$, we can see that $q+{ }_{Q_{k}} r=$ $q+Q_{t} r$ and $q \cdot Q_{k} r=q \cdot Q_{t} r$, for any $t \geq k$. Therefore, $(p, q, r, t) \in P$ and $(v, q, r, t) \in T$ if and only if $t \geq k, q+{ }_{Q_{k}} r=p$ and $q \cdot Q_{k} r=v$. Note that if $p \notin Q_{k}$ or $v \notin Q_{k}$, the corresponding pairs are not in $P$ or $T$ respectively.

We argue that Construction 4.19 is computable. Construction 4.19 appeals to Corollaries 3.19 and 3.21. By Remarks 3.20 and 3.22 these results are effective, which means there is an effective procedure for passing from effective descriptions of the inputs to effective descriptions of the outputs.

We need to argue that the function taking $k$ to:

(i) $a_{\sigma_{k}}, b_{\sigma_{k}}$,

(ii) a computable index for $Q_{k}$,

(iii) a computable index for $R_{k}$,

(iv) a computable index for the isomorphism between $R_{k}[y, \bar{x}]$ and $Q_{k}$, is computable. This is done by induction on $k$.

In particular, at Step 0 we take a computable set $Q_{0}$ and put a ring structure on it such that $Q_{0} \cong \mathbb{Q}$. Step 1 describes a process that takes the computable ring $Q_{0}$ and its computable superset $Q_{1}$ and outputs a computable ring structure on $Q_{1}$ and an element $a \in Q_{1}$ transcendental over $Q_{0}$ such that $Q_{1}=Q_{0}[a]$. These steps are executed once, so uniformity is not an issue.

Assume this has been done up to $k$. We can effectively tell from Construction 4.19 what $\sigma_{k+1}$ is, and so which of the two cases holds. In each case we explain how to effectively get (i), (ii), (iii) and (iv).

Step $k+1$, in the first case $T_{k+1}=T_{k}, \sigma_{k+1}=\sigma_{k}^{-}$; we first find the greatest $m<k$ such that $\sigma_{k+1}=\sigma_{m}$.

(i): $a_{\sigma_{k+1}}=a_{\sigma_{m}}$ and $b_{\sigma_{k+1}}=b_{\sigma_{m}}$, which by induction we already have.

(ii): first note that by induction we have an index for a computable isomorphism between $Q_{k}$ and $R_{k}[y, \bar{x}]$ over $R_{k}$. The argument given in the construction shows how to effectively obtain a computable index for the set $\left\{\langle c, m\rangle \in Q_{k} \times \omega\left|b_{\sigma_{k}}^{m}\right| c\right\}$. We have a computable index for the set $Q_{k+1}$ since $\left\langle Q_{n}\right\rangle$ is uniformly computable by Proposition 4.18, a computable index for 
$R_{k}$ by induction, and we have $b_{\sigma_{k}}$ also by induction. Then we have all the inputs needed for Corollary 3.19 and we effectively get an index for $Q_{k+1}$ from Remark 3.20.

(iii): we have indices for $R_{k}, Q_{k}$ by induction, and $Q_{k+1}$ obtained previously, we have $b_{\sigma_{k}}$ and indeed the sequence $a, \bar{b}$ also by induction. By Remark 3.26, we get effectively a computable index for $R_{k+1}$.

(iv): an index for an isomorphism from $R_{k+1}[y, \bar{x}]$ to $Q_{k+1}$ is found effectively given indices for $R_{k+1}, Q_{k+1}$ and given $a_{\sigma_{k+1}}, b_{\sigma_{k+1}\left\lceil 1,2 \cdots\left|\sigma_{k+1}\right|\right.}$ which we already have. This is done by observing that Remark 3.8 is uniform: given indices for $R$ and $Q$, and given $\bar{a}$, we get an index for the map $\langle f, \bar{a}\rangle \mapsto f(\bar{a})$.

Finally, step $k+1$, in the second case $T_{k+1}=T_{k} \cup\left\{\sigma_{k+1}\right\}$, and $\sigma_{k+1}$ is new.

(i) and (ii): by induction we have indices for $R_{k}$ and $Q_{k}$, and we have $a, \bar{b}$. This gives us a computable index for $R_{k}[\bar{b}]$ by Remark 3.24. We have an index for the set $Q_{k+1}$. So by Remark 3.22, we effectively get an index for $Q_{k+1}$, and also we get the elements $a_{\sigma_{k+1}}$ and $b_{\sigma_{k+1}}$.

(iii): $R_{k+1}=R_{k}$ and by induction we have an index for $R_{k}$.

(iv): exactly as above.

Computability of $Q_{\omega}$ follows from Proposition 4.24.

Proposition 4.25. The ring $Q_{\omega}$ is computable.

Proof. Given $q, r \in Q_{\omega}$, by Proposition 4.24, the sets $\left\{(q, n) \mid q \in Q_{n}\right\}$, $\left\{(p, q, r, k) \mid p=q+{ }_{Q_{k}} r\right\}$ and $\left\{(p, q, r, k) \mid p=q \cdot Q_{k} r\right\}$ are computable.

So one can find $k_{1}, k_{2} \in \omega$, such that $q \in Q_{k_{1}}$ and $r \in Q_{k_{2}}$. If $k=\max \left(k_{1}, k_{2}\right)$, then because $Q_{k}<Q_{\omega}, q+Q_{\omega} r=q+Q_{k} r$ and $q \cdot Q_{\omega} r=q \cdot Q_{k} r$.

We will observe that $b_{\sigma}$ is invertible in $Q_{\omega}$ if $\sigma$ is terminal in $T$. Further, an element will be invertible in $Q_{\omega}$ if it was explicitly made invertible in a ring $Q_{n}$ in the sequence.

Remark 4.26. $p$ is invertible in $Q_{\omega}$ if and only if it is invertible in $Q_{n}$, for some $n \in \omega$. Furthermore, if $p$ is invertible in $Q_{n}$, then it is invertible in all $Q_{m}$ with $m \geq n$. 
Proof. Suppose $p \cdot Q_{\omega} p^{-1}=1$. Then $p \cdot Q_{n} p^{-1}=1$ in some $Q_{n}$.

Since $Q_{n}<Q_{m}$ for all $m>n, p, p^{-1} \in Q_{m}$ and furthermore $p \cdot Q_{m} p^{-1}=1$.

As we noted above, if $\sigma$ is terminal there will be a stage $k$ of Construction 4.19 at which we make $b_{\sigma}$ a unit of $Q_{k+1}$.

Lemma 4.27. Suppose $\sigma$ is terminal in $T$. Then there is some stage s such that $b_{\sigma}$ is a unit of $Q_{s+1}$.

Proof. Proposition 4.13 together with the fact that $\sigma$ is terminal imply there exist stages $s$ with $s \geq k$ such that $\sigma_{s+1}$ does not extend $\sigma$.

Choose $s \geq k$ to be the least such stage.

By the choice of $s$, we have in Construction 4.3: $\sigma_{s}=\sigma_{k}$ and $\sigma_{s+1}=\sigma_{k}^{-}$.

This means $b_{\sigma_{k}}$ is made a unit of $Q_{s+1}$.

The units $b_{\sigma}$ of $Q_{\omega}$ are precisely the $b$ elements indexed by terminal elements of $T$.

Lemma 4.28. $b_{\sigma}$ is a unit of $Q_{\omega}$ if and only if $\sigma$ is terminal in $T$.

Proof. Suppose $\sigma$ is terminal. By Proposition 4.27, there is stage $k$ for some $k \in \omega$ such that $b_{\sigma}$ is a unit of $Q_{k}$. Since $Q_{k}<Q_{\omega}, b_{\sigma}$ is a unit of $Q_{\omega}$.

Conversely, suppose $\sigma$ is non-terminal in $T$. Let $k$ be the step at which $\sigma$ is added to the union, so $\sigma_{k}=\sigma$, so we have the ring $Q_{k}=$ $R_{k}\left[a_{\sigma_{k}}, b_{\sigma_{k} \backslash n}\right]_{n=1,2 \cdots\left|\sigma_{k}\right|}$ where the presented variables form an independent set over $R_{k}$.

Since $\sigma$ is non-terminal, by Remark 4.13, for all $n>k, \sigma_{n} \succeq \sigma_{k}=\sigma$ and thus $\sigma$ is an initial segment of $\sigma_{n}$, and so, in $Q_{n}, b_{\sigma}$ is transcendental over $R_{n}$, since all initial segments $\sigma$ of $\sigma_{n}$ index elements $b_{\sigma}$ in the independent set that generates $R_{n}$.

Therefore, by Remark 2.48, $b_{\sigma}$ is not invertible in $Q_{n}$. By Remark $4.26, b_{\sigma}$ is not invertible in $Q_{\omega}$.

The structure of the ascending chain of rings $\left\langle Q_{i}\right\rangle_{i \in \omega}$ makes it possible to factorize an element $a_{\sigma}$ using elements $a_{\tau}$ and $b_{\tau}$ where $\tau$ are strings descending from $\sigma$. This will produce an infinite descending chain in divis- 
ibility, along the infinite path of $T$. We refer to these factorizations in the following propositions.

Proposition 4.29. If $\tau \prec \sigma_{n}$ and $\tau_{0}, \tau_{1} \cdots \tau_{t} \in T$ is the sequence such that $\tau_{0}=\tau, \tau_{t}=\sigma_{n}$ and $\tau_{i}=\tau_{i+1}^{-}$for $0 \leq i<t$, then $a_{\tau}=a_{\sigma_{n}} \prod_{i=1}^{t} b_{\tau_{i}}$ in $Q_{n}=$ $R_{n}\left[a_{\sigma_{n}}, b_{\sigma_{n}\lceil m}\right]_{m=1,2 \cdots\left|\sigma_{n}\right|}$.

Proof. For each $i \in\{1,2 \cdots t\}$, let $n(i)$ be the least such that $\tau_{i}=\sigma_{n(i)}$. Then by Construction 4.19 , in $Q_{n(i)}, a_{\tau_{i-1}}=a_{\tau_{i}} b_{\tau_{i}}$. Note that $n(i) \leq n$ for each $i$, so $a_{\tau_{i-1}}=a_{\tau_{i}} b_{\tau_{i}}$ in $Q_{n}$ as well. By induction on $k \leq t$ we see that in $Q_{n}$, $a_{\tau}=a_{\tau_{k}} \prod_{i=1}^{k} b_{\tau_{i}}$.

Proposition 4.30. If $\tau \prec \sigma$ and $\tau_{0}, \tau_{1} \cdots \tau_{t} \in T$ is the sequence such that $\tau_{0}=\tau, \tau_{t}=\sigma$ and $\tau_{i}=\tau_{i+1}^{-}$for $0 \leq i<t$, then $a_{\tau}=a_{\sigma} \prod_{i=1}^{t} b_{\tau_{i}}$ in $Q_{\omega}$.

Proof. Let $\sigma=\sigma_{n}$ for some $n \in \omega$. By Proposition 4.29, $a_{\tau}=a_{\sigma} \prod_{i=1}^{t} b_{\tau_{i}}$.

Intuitively, for all elements $\sigma$ terminal in $T$, we make $b_{\sigma}$ a unit. This will make $a_{\sigma}$ and its terminal ancestors associates, while also providing an associate $a_{\tau}$ for $a_{\sigma}$, where $\tau$ belongs to the infinite path. We use this trick to ensure the non-wellfoundedness of divisibility in $Q_{\omega}$.

Lemma 4.31. If $\sigma, \sigma^{\wedge} k \in T$ are non-terminal and $\tau \succcurlyeq \sigma^{\wedge} 0, k>0$, then $a_{\tau} \sim a_{\sigma}$ in $Q_{\omega}$.

Proof. Let $\tau_{0}, \tau_{1} \cdots \tau_{k} \in T$ be the finite sequence such that $\tau_{0}=\tau, \tau_{k}=\sigma$ and $\tau_{i+1}=\tau_{i}^{-}$for $0 \leq i \leq k-1$.

From Proposition 4.30, $a_{\sigma}=a_{\tau} \prod_{i=0}^{k-1} b_{\tau_{i}}$.

Since $\tau_{i} \succeq \sigma^{\frown} 0$, by Proposition 4.10, $\tau_{i}$ is terminal for all $0 \leq i<k$. By Lemma $4.28, b_{\tau_{i}}$ are all invertible in $Q_{\omega}$.

The fact that the product of finitely many units is a unit is proved by $\Sigma_{1}$ induction. 
No $a_{\sigma}$ in $Q_{\omega}$ is a unit or irreducible. Hence $a_{\lambda}$ does not have an irreducible factorization.

Proposition 4.32. For any $\sigma \in T, a_{\sigma}$ is not a unit of $Q_{\omega}$.

Proof. Suppose some $a_{\sigma}$ is a unit of $Q_{\omega}$, with $\sigma=\sigma_{m}$. Then by Remark 4.26, it is a unit of some element of the sequence $\left\langle Q_{i}\right\rangle_{i \in \omega}, Q_{k}=$ $R_{k}\left[a_{\sigma_{k}}, b_{\sigma_{k} \mid n}\right]_{n=1,2 \cdots\left|\sigma_{k}\right|}$. We can choose $k$ such that $k>m$.

By Lemma 4.31, if $\sigma$ is terminal, then $a_{\sigma} \sim a_{\tau}$ such that $\tau$ is non-terminal; here, $\tau$ is the longest non-terminal initial-segment of $\sigma . \tau$ exists by Lemma 4.11 .

So we may assume that $\sigma$ is non-terminal.

By Remark 4.13, $\sigma_{k} \succeq \sigma$, and so by Proposition $4.30 a_{\sigma_{k}} \mid a_{\sigma}$. But then $a_{\sigma_{k}}$ is a unit, but this is impossible due to Remark 2.48.

Lemma 4.33. For all $\sigma \in T$, no $a_{\sigma}$ is irreducible in $Q_{\omega}$.

Proof. In the first case, if $\sigma$ is non-terminal, by Proposition 4.15 there is some $\tau$ non-terminal such that $\sigma \prec \tau$. But then by Remark 4.30, $a_{\tau} \mid a_{\sigma}$. But we know $a_{\tau}$ cannot be a unit by Proposition 4.32 , and we know $b_{\tau}$ is also not a unit by Lemma 4.28 , and so the division $a_{\tau} \mid a_{\sigma}$ must be proper.

If $\sigma$ is terminal, then we can find the longest initial segment $\tau$ of $\sigma$ that is not. By Lemma 4.31, there is invertible $b \in Q_{\omega}$ such that $a_{\tau} b=a_{\sigma}$. This reduces the proof to the first case.

Since every ring $Q_{i}$ in our sequence is a UFD, we will use unique factorization to our advantage in inferring that all factorizations must be along a path in $T$. We give the consequence of unique factorization here.

Proposition 4.34. Let $\sigma \in T$. If $\sigma$ is non-terminal and $a_{\sigma} \in Q_{k}$, then the only irreducible factorization of $a_{\sigma}$ in $Q_{k}$ is $\left[a_{\sigma_{k}}, b_{\sigma_{k}\lceil n}|n=| \sigma|+1,| \sigma|+2 \cdots| \sigma_{k} \mid\right]$, where $Q_{k}=R_{k}\left[a_{\sigma_{k}}, b_{\sigma_{k} \mid n}\right]_{n=1,2 \cdots\left|\sigma_{k}\right|}$.

Proof. In this proof, irreducible will mean irreducible in $Q_{k}$.

Note that by Remark 4.13, $\sigma \preceq \sigma_{k}$.

By Remark 2.50, the elements $a_{\sigma_{k}}$ and $b_{\sigma_{k} \backslash n}$ are irreducible. 
By Proposition 4.30, $a_{\sigma}=a_{\sigma_{k}} \prod_{n=|\sigma|+1}^{\left|\sigma_{k}\right|} b_{\sigma_{k}\lceil n}$.

By Proposition 4.21, $Q_{k}$ is a UFD, so any factorization of $a_{\sigma}$ into irreducibles must be equal up to association to $\left[a_{\sigma_{k}}, b_{\sigma_{k} \uparrow n}|n=| \sigma|+1,| \sigma \mid+\right.$ $\left.2 \cdots\left|\sigma_{k}\right|\right]$.

As note above, $a_{\lambda}$ has no irreducible factorization. This implies that $Q_{\omega}$ is non-Atomic.

Corollary 4.35. $Q_{\omega}$ is non-Atomic.

Proof. We claim $a_{\lambda}$ has no irreducible factorization in $Q_{\omega}$. Suppose the contrary, and let $\left[p_{i} \mid i \leq n\right]$ be such a factorization. Find the minimum $k$ such that $Q_{k}$ contains all $p_{i}$ with $i \leq n$. We know $Q_{k}=R_{k}\left[a_{\sigma_{k}}, b_{\sigma_{k} \mid n}\right]_{n=1,2 \cdots\left|\sigma_{k}\right|}$.

Note the mentioned elements are irreducible in $Q_{\omega}$, we argue by contrapositive they are irreducible in $Q_{k}$. Note that $a_{\sigma_{k}}$ divides $a_{\lambda}$ in $Q_{k}$. Because $a_{\sigma_{k}}$ is a prime element of $Q_{k}$, there is some $i$ such that $a_{\sigma_{k}}$ divides $p_{i}$ in $Q_{k}$, and so divides it in $Q_{\omega}$ as well; but $a_{\sigma_{k}}$ is reducible in $Q_{\omega}$, and so $p_{i}$ too is reducible in $Q_{\omega}$.

We need the following Lemma for proving a few of the upcoming results. It essentially states that along the chain of rings $\left\langle Q_{i}\right\rangle_{i \in \omega}$, the irreducibles remain irreducible or become units.

Lemma 4.36. If $p \in Q_{n}$ is irreducible and $a_{\sigma_{n}} \nmid_{Q_{n}} p$, then either:

1. $p \in Q_{n+1}^{\times}$or

2. $p$ is irreducible in $Q_{n+1}$ and $a_{\sigma_{n+1}} \nmid_{Q_{n+1}} p$.

Proof. We have $Q_{n}=R_{n}\left[a_{\sigma_{n}}, b_{\sigma_{n}\lceil m}\right]_{m=1,2 \cdots\left|\sigma_{n}\right|}$.

According to Construction 4.19 there are two cases to consider: $Q_{n+1}=Q_{n}\left[b_{\sigma_{n}}^{-1}\right]$ and $Q_{n+1}=Q_{n}\left[a_{\sigma_{n+1}}, b_{\sigma_{n+1}}\right]$ with $a_{\sigma_{n+1}} \cdot b_{\sigma_{n+1}}=a_{\sigma_{n}}$.

Suppose $Q_{n+1}=Q_{n}\left[b_{\sigma_{n}}^{-1}\right]=R_{n}\left[b_{\sigma_{n}}, b_{\sigma_{n}}^{-1}\right]\left[a_{\sigma_{n}^{-}}, b_{\sigma_{n}\lceil m}\right]_{m=1,2 \cdots\left|\sigma_{n}\right|-1}$.

By Proposition 2.39, $Q_{n+1}$ is isomorphic to the localization of $Q_{n}$ by $\left\{b_{\sigma_{n}}^{t} \mid t \in \omega\right\}$, and by Lemma 2.23 and the fact that $Q_{n}$ is a UFD we have that $p$ is either prime or unit in $Q_{n+1}$. 
Now suppose $Q_{n+1}=Q_{n}\left[a_{\sigma_{n+1}}, b_{\sigma_{n+1}}\right]$ where $a_{\sigma_{n+1}} \cdot b_{\sigma_{n+1}}=a_{\sigma_{n}}$.

Write $a_{1}=a_{\sigma_{n+1}}, b_{1}=b_{\sigma_{n+1}}, a_{2}=a_{\sigma_{n}}, \bar{b}=b_{\sigma_{n}\lceil m}$ for $m=1,2 \cdots\left|\sigma_{n}\right|$, $R=R_{n}[\bar{b}]$ and $\bar{y}=y_{1}, y_{2} \cdots y_{\left|\sigma_{n}\right|}$.

We have $Q_{n}=R\left[a_{2}\right]$ with $a_{2}$ transcendental over $R$ and $Q_{n+1}=R\left[a_{1}, b_{1}\right]$ with $\left\{a_{1}, b_{1}\right\}$ independent over $R$ and $a_{1} \cdot b_{1}=a_{2}$.

By Proposition 2.44, let $\varphi: R[y, z] \rightarrow Q_{n+1}$ be an isomorphism over $R$, with $\varphi(y)=a_{1}$ and $\varphi(z)=b_{1}$. Similarly, let $\psi: R[x] \rightarrow Q_{n}$ be an isomorphism over $R$ with $\psi(x)=a_{2}$.

Let $h \in R[x]$ with $h=\psi^{-1}(p)$, so $h\left(a_{2}\right)=p$. Since $p$ is irreducible in $Q_{n}, h$ is irreducible in $R[x]$. Since $a_{2}$ does not divide $p$ in $Q_{n}, x=\psi^{-1}\left(a_{2}\right)$ does not divide $h$ in $R[x]$. This means that the constant coefficient of $h$ is nonzero. Let $\hat{h}=h(y z) \in R[y, z]$. Since $a_{2}=a_{1} b_{1}$ and $h\left(a_{2}\right)=p$, we see that $\hat{h}\left(a_{1}, b_{1}\right)=p$. Hence $\hat{h}=\varphi^{-1}(p)$.

We show that $p$ is irreducible in $Q_{n+1}$. To do that, let $s, t \in Q_{n+1}$ and suppose that $p=s \cdot t$; we need to show that either $s$ or $t$ is a unit of $Q_{n+1}$. Let $f=\varphi^{-1}(s)$ and $g=\varphi^{-1}(t)$; equivalently, we need to show that either $f$ or $g$ is a unit of $R[y, z]$. Since $p=s t$, applying the isomorphism $\varphi^{-1}$ we have $\hat{h}=f g$.

There are several cases to consider:

(i) $\operatorname{deg}(h)=0$,

(ii) $\operatorname{deg}_{y}(f), \operatorname{deg}_{y}(g)>0$ (this case is analogous to $\operatorname{deg}_{z}(f), \operatorname{deg}_{z}(g)>0$, which we therefore omit),

(iii) $\operatorname{deg}_{y}(f)=\operatorname{deg}_{z}(f)=0$, i.e. $f \in R$ (analogous to $g \in R$ ), and

(iv) $\operatorname{deg}_{y}(f), \operatorname{deg}_{z}(g)>0, \operatorname{deg}_{z}(f)=\operatorname{deg}_{y}(g)=0$ (analogous to the case with $y$ and $z$ exchanged).

Case (i): in this case $h=\hat{h} \in R$, and therefore $f, g \in R$ also. Since $h$ is irreducible in $R[x]$, it is irreducible in $R$, and so $f g=\hat{h}=h$ implies that either $f$ or $g$ is a unit of $R$ and certainly this implies that $f$ or $g$ is a unit of $R[y, z]$.

Case (ii): write $f=\sum_{i=0}^{e} f_{i}(z) y^{i}$ and $g=\sum_{i=0}^{d} g_{i}(z) y^{i}$, for $d, e \in \omega$ and $g_{i}, f_{i} \in$ $R[z]$ and $f_{e}, g_{d} \neq 0$.

By Proposition 2.34, there is some $\alpha \in R^{\times}$such that $f_{e}(\alpha) \neq 0$ and 
$g_{d}(\alpha) \neq 0$. Let $\bar{f}(x)=f\left(x \alpha^{-1}, \alpha\right)=\sum_{i=0}^{e} \alpha^{-i} f_{i}(\alpha) x^{i}$ and similarly let $\bar{g}(x)=$ $g\left(x \alpha^{-1}, \alpha\right)=\sum_{i=0}^{d} \alpha^{-i} g_{i}(\alpha) x^{i}$. Since $f_{e}(\alpha) \neq 0$ and $g_{d}(\alpha) \neq 0$, we have that $\operatorname{deg}_{x}(\bar{f})=e>0$ and $\operatorname{deg}_{x}(\bar{g})=d>0$. Since the units of $R[x]$ are the same as the units of $R$ by Proposition 2.30, neither $\bar{f}$ nor $\bar{g}$ are units of $R[x]$.

We have $h=h\left(x \alpha^{-1} \cdot \alpha\right)=\hat{h}\left(x \alpha^{-1}, \alpha\right)=\bar{f} \bar{g}$ in $R[x]$. So in $R[x] h$ is the product of two non-units, which contradicts the assumption that it is an irreducible. Thus, case (ii) cannot happen.

Case (iii): if $f \in R$, then the monomials which appear in $g$ are the same as the monomials which appear in $\hat{h}=f g$. Write $h=\sum_{i=0}^{v} h_{i} x^{i}$ with $h_{i} \in R$ and $h_{v} \neq 0$; then $\hat{h}=\sum_{i=0}^{v} h_{i} y^{i} z^{i}$, and so $g=\sum_{i=0}^{v} \gamma_{i} y^{i} z^{i}$, with $h_{i}=f \cdot \gamma_{i}$ for all $i$.

Let $\bar{g}=\sum_{i=0}^{v} \gamma_{i} x^{i} \in R[x]$; so $h=f \bar{g} \in R[x]$. We assume that case (i) does not hold, and so $v>0$, and so $\bar{g}$ is not a unit of $R[x]$. Since $h$ is irreducible in $R[x]$, it follows that $f$ is a unit of $R[x]$, hence a unit of $R$, and so is also a unit of $R[y, z]$.

Case (iv): this is the interesting case, in which we use the assumption that $x$ does not divide $h$. In this case $f \in R[y]$ and $g \in R[z]$ are nonconstant. The constant coefficient of $\hat{h}$ is the same as the constant coefficient $h_{0}$ of $h$, which by assumption is nonzero. Since $f g=\hat{h}$, this implies that both the constant coefficient $f_{0}$ of $f$ and the constant coefficient $g_{0}$ of $g$ are nonzero, as $h_{0}=f_{0} g_{0}$. Let $d=\operatorname{deg}_{y}(g)$, and let $f_{d}$ be the leading coefficient of $f$. Then $g_{0} f_{d} z^{d}$ is a monomial of $f g=\hat{h}$, which is impossible, since the monomials of $\hat{h}$ are all of the form $h_{i} y^{i} z^{i}$, for $h_{i} \in R$. Thus case (iv) cannot happen either.

We still need to show that $a_{\sigma_{n+1}} \nmid p$.

Again, we consider the cases $Q_{n+1}=Q_{n}\left[b_{\sigma_{n}}^{-1}\right]$ and $Q_{n+1}=Q_{n}\left[a_{\sigma_{n+1}}, b_{\sigma_{n+1}}\right]$.

If $Q_{n+1}=Q_{n}\left[b_{\sigma_{n}}^{-1}\right]$, then $\sigma_{n+1}=\sigma_{n}^{-}$, write $a_{1}=a_{\sigma_{n+1}}, b_{1}=b_{\sigma_{n+1}}, a_{2}=$ $a_{\sigma_{n}}, b_{2}=b_{\sigma_{n}}$.

Note that $a_{2} b_{2}=a_{1}$ so $a_{2} b_{2} \nmid p$ in $Q_{n}$. Suppose $a_{1} \mid p$ in $Q_{n+1}$, so there is $\gamma \in Q_{n+1}$ such that $a_{1} \cdot \gamma=p$, so $a_{2} b_{2} \cdot \gamma=p$. By Proposition $2.38, \gamma=c / b_{2}^{k}$ for some $c \in Q_{n}, k \in \omega$. Then, $a_{2} b_{2} c=b_{2}^{k} p$, so $a_{2} \mid b_{2}^{k} p$ in $Q_{n}$. By Proposition 2.50, 
$a_{2}$ is irreducible in $Q_{n}$, by Proposition $4.21 Q_{n}$ is a UFD, so by Proposition $2.10 a_{2}$ is prime in $Q_{n}$. Which means $a_{2} \mid b_{2}^{k}$ or $a_{2} \mid p$ in $Q_{n}$. The second is impossible by assumption, so $a_{2} \mid b_{2}^{k}$ in $Q_{n}$, so $a_{2} \mid b_{2}$. By Proposition 2.50, $b_{2}$ is irreducible in $Q_{n}$, so either $a_{2}$ is a unit of $Q_{n}$ or $a_{2}=b_{2}$. The first case is impossible by Proposition 2.48, and the second is impossible because $a_{2}$ is transcendental over $R$, by Construction 4.19 .

Now suppose $Q_{n+1}=Q_{n}\left[a_{\sigma_{n+1}}, b_{\sigma_{n+1}}\right]$, where $\left\{a_{\sigma_{n+1}}, b_{\sigma_{n+1}}\right\}$ is independent over $Q_{n}$ and $a_{\sigma_{n+1}} \cdot b_{\sigma_{n+1}}=a_{\sigma_{n}}$. If $a_{\sigma_{n+1}} \mid p$ in $Q_{n+1}$, then $a_{\sigma_{n+1}} \gamma=p$ for some $\gamma \in Q_{n+1}^{\times}$. From Construction 4.19, $Q_{n+1}^{\times}=Q_{n}^{\times}$, so $p \gamma^{-1} \in Q_{n}$, which means $a_{\sigma_{n+1}} \in Q_{n}$, a contradiction.

If an element is irreducible or prime in a ring in the sequence $\left\langle Q_{i}\right\rangle_{i \in \omega}$, then it is either a unit or an irreducible/prime in $Q_{\omega}$.

Lemma 4.37. If $p$ is prime in some $Q_{n}$ and $p \notin Q_{\omega}^{\times}$, with $a_{\sigma_{n}} \nmid_{Q_{n}} p$, then $p$ is prime in $Q_{\omega}$ and no $\left.a_{\sigma}\right|_{Q_{\omega}} p$, for any $\sigma \in T$.

Proof. By induction on $m \geq n$ we see that $p$ is prime in $Q_{m}$ and $a_{\sigma_{m}}$ does not divide $p$ in $Q_{m}$. For if this holds for $Q_{m}$, then by Lemma 4.36 it holds for $Q_{m+1}$, unless $p$ is a unit of $Q_{m+1}$. But in that case, $p$ is a unit of $Q_{\omega}$, which we assumed is not the case.

Now let $c, d \in Q_{\omega}$ and suppose that $p \mid c d$ in $Q_{\omega}$. Let $m \geq n$ be sufficiently large so that $c, d \in Q_{m}$ and $p \mid c d$ in $Q_{m}$. Then $p \mid c$ or $p \mid d$ in $Q_{m}$, and so $p \mid c$ or $p \mid d$ in $Q_{\omega}$. Hence $p$ is prime in $Q_{\omega}$.

Let $\sigma \in T$, and suppose that $a_{\sigma} \mid p$ in $Q_{\omega}$. Since $a_{\sigma} \sim a_{\tau}$ for some nonterminal string $\tau$ by Lemma 4.31, we may assume that $\sigma$ is non-terminal. Let $m \geq n$ be sufficiently large so that $\sigma \in T_{m}$ and $a_{\sigma}$ divides $p$ in $Q_{m}$. By Proposition $4.294 .25, a_{\sigma_{m}}$ divides $a_{\sigma}$ in $Q_{m}$, and so $a_{\sigma_{m}}$ divides $p$ in $Q_{m}$, contrary to what we just showed.

Any elements of $Q_{\omega}$ that are not divisible by an $a_{\sigma}$ admit a prime factorization in $Q_{\omega}$.

Proposition 4.38. If $p \in Q_{\omega}$ such that $a_{\sigma} \nmid_{Q_{\omega}} p$ for all $\sigma \in T$, then $p$ has a prime decomposition in $Q_{\omega}$. 
Proof. If $p \in Q_{n}$ then $a_{\sigma_{n}} \nmid_{Q_{n}} p$. Since $Q_{n}$ is a UFD, by Proposition $2.10 p$ has a prime decomposition in $Q_{n}, B=\left[p_{i} \mid i \leq t\right]$.

By Lemma 4.37 and since $a_{\sigma_{n}} \nmid p$ so $a_{\sigma_{n}} \nmid p_{i}$, each $p_{i}$ is a prime or a unit of $Q_{\omega}$.

In a ring, a sequence $\left(n_{i}\right)_{i \in \omega}$ stabilizes if there exists $k^{*} \in \omega$ such that for all $k \geq k^{*}, n_{k+1}=n_{k}$. If rather $n_{k+1}=u n_{k}$ for some unit $u$, we say the sequence stabilizes up to association. If the ring is $\omega$, we say the sequence is increasing if $n_{k+1}>n_{k}$ for all $k \in \omega$. We say the sequence is non-increasing if $n_{k+1} \leq n_{k}$ for all $k \in \omega$.

Proposition 4.39. Let $\left(n_{i}\right)_{i \in \omega}$ be a non-increasing sequence in $\omega$. Then, this sequence stabilizes.

Proof. Suppose the sequence does not stabilize, so it has a subsequence $\left(m_{j}\right)_{j \in \omega}$ such that $m_{k} \neq m_{t}$ for $k \neq t$. Then, for $k>1, m_{k}<n_{0}, m_{k+1}<$ $n_{0}-1 \cdots m_{k+n_{0}}<0$, a contradiction.

If an element $a_{\sigma}$ is in $Q_{n}$ and $\sigma$ is a descendant of $\sigma_{n}$ in $T$, then $a_{\sigma}$ and the generator $a_{\sigma_{n}}$ of $Q_{n}$ are associates in $Q_{n}$.

Lemma 4.40. If $a_{\sigma} \in Q_{n}$ and $\sigma_{n} \prec \sigma$ in $T$, then $a_{\sigma_{n}} \sim a_{\sigma}$ in $Q_{n}$.

Proof. Let $\sigma \in T$. If $\sigma \in T_{m+1} \backslash T_{m}$ for some $m \geq n$, then $a_{\sigma} \in Q_{m+1} \backslash Q_{m}$. Hence, if $a_{\sigma} \in Q_{n}$ then $\sigma \in T_{n}$.

Suppose that $\sigma_{n} \prec \sigma$. Let $k$ be the least such that $\sigma=\sigma_{k}$; since $\sigma \in T_{n}$ we have $k \leq n$, and since $\sigma \neq \sigma_{n}$ we have $k \neq n$, so $k<n$.

Let $s$ be the least such that $\sigma_{s}$ does not extend $\sigma$; then $s \leq n$. Then $\sigma_{s-1}=\sigma$ and $\sigma_{s}=\sigma^{-}$, and $b_{\sigma}$ is a unit of $Q_{s}$. Hence $b_{\sigma}$ is a unit of $Q_{n}$.

Now let $\sigma \in T_{n}$ properly extending $\sigma_{n}$, and write $\sigma_{n}=\tau_{0}, \tau_{1}, \cdots \tau_{t}=\sigma$, with $\tau_{i}=\tau_{i+1}^{-}$. By Proposition 4.29, $a_{\sigma_{n}}=a_{\sigma} \prod_{i=1}^{t} b_{\tau_{i}}$ in $Q_{k}$, where $\sigma \in T_{k} \backslash T_{k-1}$. Since $k<n$, this equation holds in $Q_{n}$ as well, and by the argument just given, each $b_{\tau_{i}}$ for $i \geq 1$ is a unit of $Q_{n}$, and so $a_{\sigma} \sim a_{\sigma_{n}}$ in $Q_{n}$.

The generator $a_{\sigma_{n}}$ of $Q_{n}$ divides in $Q_{n}$ any element $a_{\sigma}$ that belongs to $Q_{n}$. 
Proposition 4.41. If $a_{\sigma} \in Q_{n}$, then $\left.a_{\sigma_{n}}\right|_{Q_{n}} a_{\sigma}$.

Proof. There are three cases to consider:

(i) $\sigma \preceq \sigma_{n}$,

(ii) $\sigma_{n} \prec \sigma$ and

(iii) $\sigma \mid \sigma_{n}$.

If (i) $\sigma \preceq \sigma_{n}$, by Proposition 4.29, the result follows.

If (ii) $\sigma_{n} \prec \sigma$, by Lemma 4.40 , the result follows.

If (iii) $\sigma \mid \sigma_{n}$, by Proposition $4.13 \sigma$ is terminal. Take the longest nonterminal $\tau$ such that $\tau \prec \sigma$ and $\tau \preceq \sigma_{n}$. Since $\sigma \in T_{n}$, $\tau$ is the longest non-terminal initial segment of $\sigma$.

By Lemma 4.40, if $\tau=\sigma_{m}$ with $\sigma \in T_{m}, a_{\tau}$ and $a_{\sigma}$ are associates in $Q_{m}$ with $Q_{m}<Q_{n}$, hence they associate in $Q_{n}$.

By Proposition $4.29, a_{\sigma_{n}} \mid a_{\tau}$. The result follows by transitivity.

If $d \in Q_{n}$ and the generator $a_{\sigma_{n}}$ of $Q_{n}$ does not divide $d$, it follows that no other $a$ element divides $d$ in $Q_{\omega}$.

Proposition 4.42. Let $Q_{n}$ be a ring in the sequence $\left(Q_{i}\right)_{i \in \omega}$. If for some $d \in$ $Q_{n}, a_{\sigma_{n}} \nmid_{Q_{n}} d$, then for all $\delta \in T a_{\delta} \nmid_{Q_{\omega}} d$.

Proof. Suppose $\left.a_{\delta}\right|_{Q_{\omega}} d$. Then $\left.a_{\delta}\right|_{Q_{m}} d$ for some $m \in \omega$ such that $m>n$. Since $a_{\delta} \in Q_{m}$, by Proposition $\left.4.41 a_{\sigma_{m}}\right|_{Q_{m}} a_{\delta}$, so $\left.a_{\sigma_{m}}\right|_{Q_{m}} d$.

Let $d=u p_{1} p_{2} \cdots p_{t}$ be an irreducible factorization of $d$ in $Q_{n}$. Since $a_{\sigma_{n}} \nmid_{Q_{n}}$ $d$, we have $a_{\sigma_{n}} \nmid_{Q_{n}} p_{i}$ for $1 \leq i \leq t$, and by iterating Lemma 4.36 each $p_{i}$ is either a unit of $Q_{m}$ or irreducible in $Q_{m}$ with $a_{\sigma_{m}} \nmid_{Q_{m}} p_{i}$. Since $a_{\sigma_{m}}$ is irreducible in $Q_{m}, a_{\sigma_{m}} \nmid_{Q_{m}} d$, a contradiction.

Next, we give a general form for the elements of $Q_{\omega}$. This will be useful when dealing with infinite descending chains in divisibility.

Lemma 4.43. Every element of $Q_{\omega}$ is of the form $d a_{\sigma}^{n}$ for some $\sigma \in T, n \in \omega$ and $d \in Q_{\omega}$ not divisible by any $a_{\tau}$. 
Proof. Let $q \in Q_{\omega}$; let $m \in \omega$ such that $q \in Q_{m}$. Let $k$ be the greatest power of $a_{\sigma_{m}}$ which divides $q$ in $Q_{m}$; so $d=q / a_{\sigma_{m}}^{k} \in Q_{m}$ and $a_{\sigma_{m}}$ does not divide $q / a_{\sigma_{m}}^{k}$ in $Q_{m}$. By Proposition 4.42, no $a_{\tau}$ divides $d$ in $Q_{\omega}$, and we have $q=d \cdot a_{\sigma_{m}}^{k}$ in $Q_{\omega}$.

By an infinite descending chain in divisibility in a ring we mean a sequence $\left(c_{i}\right)_{i \in \omega}$ such that for all $i, c_{i+1}$ properly divides $c_{i}$.

Any infinite divisibility descending sequence of $Q_{\omega}$ whose terms do not have an $a_{\sigma}$ factor must stabilize.

Lemma 4.44. Suppose $\left(c_{i}\right)_{i \in \omega}$ is a sequence of elements of $Q_{\omega}$ such that $c_{k+1} \mid c_{k}$, for all $k \in \omega$. Then, if for all $\sigma \in T, a_{\sigma} \nmid c_{0}$, there is some $k^{*} \in \omega$ such that for all $k \geq k^{*} c_{k} \sim c_{k+1}$.

Proof. Since for all $\sigma \in T a_{\sigma} \nmid c_{0}$ and every $c_{k}$ divides $c_{0}$, it follows that $a_{\sigma} \nmid c_{k}$ for all $k \in \omega$.

By Proposition 4.38, for all $k \in \omega, c_{k} \sim \prod B_{k}$ where $B_{k}=\left[p_{k, i} \mid i \leq n_{k}\right]$, for $n_{k} \in \omega$, where each $p_{k, i}$ is non-unit and prime.

By Proposition 2.12 we have $B_{k+1} \subseteq B_{k}$ up to association, and since each $B_{k}$ is finite, the sequence $\left|B_{k}\right|$ must stabilize; hence the sequence $\left(c_{i}\right)_{i \in \omega}$ stabilizes up to association.

An essential ingredient to our proof: an infinite descending chain in divisibility of $Q_{\omega}$ must compute the Halting Problem.

Proposition 4.45. Any infinite descending chain in divisibility of $Q_{\omega}$ computes $\emptyset^{\prime}$.

Proof. Let $\left(c_{i}\right)_{i \in \omega}$ be such a chain. We claim we can write non-effectively each $c_{k}$ as $c_{k}=d_{k} \prod_{b \in B_{k}} b \cdot a_{\epsilon_{k}}^{m_{k}}$, where $B_{k}$ is a multiset of elements $b_{\sigma}$, for $\sigma$ non-terminal, and $d_{k}$ is not divisible in $Q_{\omega}$ by any $a_{\sigma}$ and by any $b_{\sigma}$ with $\sigma$ non-terminal.

By Lemma 4.43 there is some $d_{k}^{\prime}$ and some $\epsilon_{k} \in T$ and $m_{k} \in \omega$ such that no $a_{\sigma}$ divides $d_{k}^{\prime}$ in $Q_{\omega}$ and such that $c_{k}=d_{k}^{\prime} a_{\epsilon_{k}}^{m_{k}}$.

By Proposition $4.38, d_{k}^{\prime}$ has a prime decomposition $D_{k}^{\prime}$ in $Q_{\omega}$. Let $B_{k}$ be the multiset of primes $p \in D_{k}^{\prime}$ such that $p \sim b_{\sigma}$ for some non-terminal $\sigma \in T$. 
Then let $d_{k}=\prod\left(D_{k}^{\prime} \backslash B_{k}\right)=d_{k}^{\prime} / \prod B_{k}$.

We claim $d_{k+1} \mid d_{k}$. Take $m$ sufficiently large so $Q_{m}$ contains $d_{k}, d_{k+1}, a_{\epsilon_{k}}, a_{\epsilon_{k+1}}$ and each element of $B_{k}$ and $B_{k+1}$ such that $\left.c_{k+1}\right|_{Q_{m}} c_{k}$ and $\sigma_{m}$ is a correct string with $\sigma_{m} \notin T_{m-1}$. Then for all $\sigma$ with $b_{\sigma} \in Q_{m}, \sigma$ is correct if and only if $\sigma \preceq \sigma_{m}$ if and only if $b_{\sigma}$ is prime in $Q_{m}$; if $b_{\sigma} \in Q_{m}$ and $\sigma \npreceq \sigma_{m}$ then $b_{\sigma}$ is a unit of $Q_{m}$.

In $Q_{m}$, let $D_{k}$ and $D_{k+1}$ be prime decompositions of $d_{k}$ and $d_{k+1}$ respectively, and let $A_{k}$ and $A_{k+1}$ be prime decompositions of $a_{\epsilon_{k}}^{m_{k}}$ and $a_{\epsilon_{k+1}}^{m_{k+1}}$ respectively. So $A_{k} \cup B_{k} \cup D_{k}$ is a a prime decomposition of $c_{k}$ in $Q_{m}$, and similarly for $k+1$. So $\left.c_{k+1}\right|_{Q_{m}} c_{k}$ means that $A_{k+1} \cup B_{k+1} \cup D_{k+1}$ is a subset of $A_{k} \cup B_{k} \cup D_{k}$ up to association. Now all the primes which appear in $A_{k} \cup B_{k}$ (and in $A_{k+1} \cup B_{k+1}$ ) are associates of either $a_{\sigma_{m}}$ or $b_{\sigma}$ for some $\sigma \preceq \sigma_{m}$, and no such primes can occur in $D_{k}$ or in $D_{k+1}$. Hence we can conclude that $D_{k+1} \subseteq D_{k}$ up to association, and so $d_{k+1} \mid d_{k}$ in $Q_{m}$, and so $d_{k+1} \mid d_{k}$ in $Q_{\omega}$. We also see that $a_{\sigma_{m}}$ does not appear in $B_{k} \cup D_{k}$ (or $B_{k+1} \cup D_{k+1}$ ) but appears (up to association) with multiplicity $m_{k}$ in $A_{k}$, and $m_{k+1}$ in $A_{k+1}$. Then the fact that $A_{k+1} \cup B_{k+1}$ is a subset of $A_{k} \cup B_{k}$ (up to association) implies that $m_{k+1} \leq m_{k}$.

Write $c_{k}^{\prime}=c_{k+K} / d_{K}$ and note that $\left\langle c_{k}\right\rangle$ computes the sequence $\left\langle c_{k}^{\prime}\right\rangle$. To avoid excess notation, write $c_{k}$ instead of $c_{k}^{\prime}$, and it remains to show that the new $\left\langle c_{k}\right\rangle$ computes $\emptyset^{\prime}$.

For the multiset $B$ of elements of $T$, if $S=\left[\sigma \mid b_{\sigma} \in B\right]$, we write $B(S)=B$.

Claim (i). Let $S$ be a finite multiset of strings from $T$ and let $\rho \in T$. We claim there exist $S^{\prime}$ and and $\rho^{\prime}$ such that:

(a) all of the strings in $S^{\prime} \cup\left[\rho^{\prime}\right]$ are comparable, and

(b) $a_{\rho^{\prime}}$ and $a_{\rho}$ are associates in $Q_{\omega}$, and

(c) $\prod_{b \in B(S)} b$ associates with $\prod_{b \in B\left(S^{\prime}\right)} b$ in $Q_{\omega}$.

Proof: let $R$ be the collection of initial segments of strings in $S \cup[\rho]$, so $R$ is a finite subtree of $T$. Let $\tau$ be the rightmost leaf of $R$. Then, every $\sigma \in R$ 
which is not extended by $\tau$ is terminal, hence by Lemma $4.28, b_{\sigma}$ is a unit of $Q_{\omega}$. We let $S^{\prime}=[\sigma \cap \tau \mid \sigma \in S]$ and let $\rho^{\prime}=\rho \cap \tau$.

Let $S$ and $S^{\prime}$ be multisets of strings of $T$ and let $\rho, \rho^{\prime} \in T$.

Claim (ii). There is a multiset $\bar{S}$ of strings of $T$ and a string $\bar{\rho} \in T$ such that:

(a) $a_{\rho^{\prime}}^{m_{k}} \cdot \prod B\left(S^{\prime}\right) \sim a_{\bar{\rho}}^{m_{k}} \prod B(\bar{S})$,

(b) for all $\sigma \in \bar{S}, \sigma \preceq \bar{\rho}$ and

(c) either $\rho^{\prime} \preceq \bar{\rho}$ or $\rho^{\prime}$ lies lexicographically to the left of $\bar{\rho}$, i.e. if $\sigma=\rho^{\prime} \cap \bar{\rho}$ then $\sigma^{\wedge} 0 \preceq \rho^{\prime}$ and $\sigma^{\wedge} s \preceq \bar{\rho}$ for some $s>0$.

Proof: let $R$ be the collection of initial segments of $S \cup S^{\prime} \cup\left[\rho, \rho^{\prime}\right]$. Let $\tau$ be the rightmost leaf of $R$. Let $\bar{\rho}=\tau$ and let $\bar{S}=\left[\sigma \cap \tau \mid \sigma \in S^{\prime}\right] \cup m_{K} \cdot[\sigma \mid$ $\left.\left(\rho^{\prime} \cap \tau\right) \prec \sigma \preceq \tau\right]$.

Using this claim we can find, computably from $\left\langle c_{k}\right\rangle$, by recursion a sequence $\left\langle S_{k}, \rho_{k}\right\rangle$ such that for all $k$ :

(a') $s_{k} \sigma_{\rho_{k}}^{m_{k}} \prod B\left(S_{k}\right)$,

(b') for all $\sigma \in S_{k}, \sigma \preceq \rho_{k}$, and

(c') either $\rho_{k} \preceq \rho_{k+1}$ or $\rho_{k}$ lies lexicographically to the left of $\rho_{k+1}$.

Let $\eta_{k}=\rho_{k} \cap \rho_{k+1}$, and let $\bar{\rho}_{k}$ be longest correct initial segment of $\rho_{k}$. Note that the sequence $\left\langle\eta_{k}\right\rangle$ is computable from $\left\langle c_{k}\right\rangle$.

Property ( $\mathrm{c}^{\prime}$ ) above implies that $\bar{\rho}_{k} \preceq \eta_{k}$ : this certainly holds if $\rho_{k} \preceq \rho_{k+1}$, otherwise, because $\rho_{k+1}$ witnesses that $\eta_{k}^{\curlyvee} 0 \preceq \rho_{k}$ is terminal. Hence $\bar{\rho}_{k} \preceq$ $\bar{\rho}_{k+1}$, as $\bar{\rho}_{k}$ is a correct initial segment of $\rho_{k+1}$.

Further, we note that property ( $\left.\mathrm{b}^{\prime}\right)$ above implies that $\rho \in S_{k}$ is correct if and only if $\rho \preceq \bar{\rho}_{k}$. Let $\bar{S}_{k}=\left[\sigma \in S_{k} \mid \sigma \preceq \bar{\rho}_{k}\right]$, then $c_{k} \sim a_{\bar{\rho}_{k}}^{m_{K}} \prod B\left(\bar{S}_{k}\right)$.

Note that in the model of $R C A_{0}$ in which we work, the sequence $\left\langle\bar{S}_{k}\right\rangle$ may not exist, but it is definable.

Claim (iii). For all $\sigma \preceq \bar{\rho}_{k}, m_{\sigma}\left(S_{k+1}\right) \leq m_{\sigma}\left(S_{k}\right)$.

Proof: let $n$ be large, so that $c_{k+1} \mid c_{k}$ in $Q_{n}$ and all the elements mentioned are in $Q_{n}$. Since $\bar{\rho}_{k+1}$ is correct, it is an initial segment of $\sigma_{n}$. Let $A_{k}$ be an irreducible factorization of $a_{\bar{\rho}_{k}}^{m_{K}}$ in $Q_{n}$, so $A_{k} \cup B\left(\bar{S}_{k}\right)$ is an irreducible factorization of $c_{k}$ in $Q_{n}$. We also have that $B\left(\bar{S}_{k+1}\right)$ is a set of 
primes of $Q_{n}$ and $\prod B\left(\bar{S}_{k+1}\right) \mid c_{k}$ in $Q_{n}$, as $\prod B\left(\bar{S}_{k+1}\right) \mid c_{k+1}$ in $Q_{n}$, and so $B\left(\bar{S}_{k+1}\right) \subseteq A_{k} \cup B\left(\bar{S}_{k}\right.$ up to association. However, for $\sigma \preceq \bar{\rho}_{k}, b_{\sigma} \notin A_{k}$, as $b_{\tau} \in A_{k}$ implies $\bar{\rho}_{k} \prec \tau$. Hence $m_{\sigma}\left(\bar{S}_{k+1}\right) \leq m_{\sigma}\left(\bar{S}_{k}\right)$. Since $\sigma \preceq \bar{\rho}_{k}, \bar{\rho}_{k+1}$, $m_{\sigma}\left(S_{k}\right)=m_{\sigma}\left(\bar{S}_{k}\right)$ and similarly for $k+1$.

Claim (iv). If $\eta_{k}$ is not correct, then there is some correct $\sigma \preceq \eta_{k}$ such that $m_{\sigma}\left(S_{k}\right)<m_{\sigma}\left(S_{k+1}\right)$.

Proof: the assumption implies that $\bar{\rho}_{k}=\bar{\rho}_{k+1}$ is a proper initial segment of $\eta_{k}$, and so

$$
a_{\bar{\rho}_{k}}^{m_{K}} \cdot \prod B\left(\bar{S}_{k}\right) \sim c_{k} \mid c_{k+1} \sim a_{\bar{\rho}_{k+1}}^{m_{K}} \cdot \prod B\left(\bar{S}_{k+1}\right)
$$

implies that

$$
\prod B\left(\bar{S}_{k}\right) \mid \prod B\left(\bar{S}_{k+1}\right)
$$

and the division is proper. However, if for all $\sigma \preceq \bar{\rho}_{k}$ we have $m_{\sigma}\left(S_{k}\right) \leq m_{\sigma}\left(S_{k+1}\right)$, then by Claim (iii) we would have $\bar{S}_{k}=\bar{S}_{k+1}$, so the division would not be proper.

Now define a string $\zeta_{k}$ as:

$\zeta_{k}=\eta_{k}$ if for all $\sigma \preceq \eta_{k}, m_{\sigma}\left(S_{k+1}\right)=m_{\sigma}\left(S_{k}\right)$. Otherwise, let $\zeta_{k}$ be the shortest initial segment $\sigma$ of $\eta_{k}$ such that $m_{\sigma}\left(S_{k+1}\right)<m_{\sigma}\left(S_{k}\right)$. Claim (iv) implies each $\zeta_{k}$ is correct. It follows that the strings $\zeta_{k}$ are pairwise comparable.

Now define the string $\mu_{k}$ as the longest string in $\left\{\zeta_{j} \mid j \leq k\right\}$. So each $\mu_{k}$ is correct and $\mu_{k} \preceq \mu_{k+1}$.

Claim (v). Suppose that $\zeta_{k}=\eta_{k}$ and $m_{\eta_{k}}\left(S_{k}\right)=m_{\eta_{k}}\left(S_{k+1}\right)$. In other words for all $\sigma \preceq \eta_{k}$ we have $m_{\sigma}\left(S_{k}\right)=m_{\sigma}\left(S_{k+1}\right)$. Then $\bar{\rho}_{k+1} \succ \eta_{k}$.

Proof: suppose for a contradiction that $\bar{\rho}_{k+1}=\eta_{k}=\bar{\rho}_{k}$. The assumption on $k$ implies that $\bar{S}_{k}=\bar{S}_{k+1}$. And then:

$$
c_{k} \sim a_{\bar{\rho}_{k}}^{m_{K}} \prod B\left(\bar{S}_{k}\right)=a_{\bar{\rho}_{k+1}}^{m_{K}} \prod B\left(\bar{S}_{k+1}\right) \sim c_{k+1},
$$

contrary to our assumption that the division $c_{k+1} \mid c_{k}$ is proper. 
Claim (vi). For all $k \in \omega$, for all $m \geq k, \bar{\rho}_{k} \preceq \rho_{m}$.

Proof: by induction on $m \geq k$. Note that the sequence $\left\langle\rho_{m}\right\rangle_{m \geq k}$ exists, and so the statement is both $\Sigma_{1}$ and $\Pi_{1}$ as $\bar{\rho}_{k}$ is fixed.

For the base case, $m=k$ and note that $\bar{\rho}_{k} \preceq \bar{\rho}_{m}$. Let $m \geq k$ and suppose $\bar{\rho}_{k} \preceq \rho_{m}$. Since $\bar{\rho}_{k}$ is correct, we have $\bar{\rho}_{k} \preceq \bar{\rho}_{m}$. We know that $\bar{\rho}_{m} \preceq \bar{\rho}_{m+1} \preceq \rho_{m+1}$ so $\bar{\rho}_{k} \preceq \rho_{m+1}$.

It follows that for all $k$, for all $m \geq k, \zeta_{k} \preceq \rho_{m}$, so $\zeta_{k} \preceq \bar{\rho}_{m}$. It then follows that for all $k, \mu_{k} \preceq \rho_{k}$, since $\mu_{k}=\zeta_{j}$ for some $j \leq k$. Since $\mu_{k}$ is correct, $\mu_{k} \preceq \bar{\rho}_{k}$.

Claim (vii). For all $\sigma \preceq \mu_{k}, m_{\sigma}\left(S_{k}\right) \geq m_{\sigma}\left(S_{k+1}\right)$.

Proof: immediate from Claim (iii), since $\mu_{k} \preceq \bar{\rho}_{k}$.

Claim (viii). Now let $k \in \omega$ and $n=\left|S_{k}\right|+3$. We claim $\left|\mu_{k+n}\right|>\left|\mu_{k}\right|$.

Proof: we see that $n>\left|S_{k}\right|+2$ together with Claim (vii) implies that there are at least two $i, j \in\{k, k+1, \cdots, k+n-1\}$ such that for all $\sigma \preceq \mu_{k}$, $m_{\sigma}\left(S_{i}\right)=m_{\sigma}\left(S_{i+1}\right)$ and similar for $j$. If $\eta_{i} \succ \mu_{k}$ then $\zeta_{i} \succ \mu_{k}$ and we are done. Otherwise, $\eta_{i}=\mu_{i}$ and by Claim (vi) $\bar{\rho}_{i+1} \prec \mu_{i}$. By Claim (vi), $\bar{\rho}_{i+1} \preceq \bar{\rho}_{j}$, and we know that $\bar{\rho}_{j} \preceq \eta_{j}$, so $\eta_{j} \succ \mu_{k}$, and so $\zeta_{j} \succ \mu_{k}$, and we are done.

Now we know that for all $i$ there is some $j>i$ such that $\mu_{i} \prec \mu_{j}$. By recursion we define a sequence $\left\langle\mu_{k}^{\prime}\right\rangle$; given $\mu_{k}^{\prime}=\mu_{i}$ for some $i$, we let $\mu_{k+1}^{\prime}=$ $\mu_{j}$ for some $j>i$ such that $\mu_{i} \prec \mu_{j}$. Then $\mu_{k}^{\prime} \prec \mu_{k+1}^{\prime}$, and so by Lemma 4.17, $\left\langle\mu_{k}^{\prime}\right\rangle$ computes $\emptyset^{\prime}$. This completes our proof.

We now put all of our previous work together to show that there exists a ring that satisfies the conditions of the contrapositive of Theorem 1.3 and some sequence in the ring computes the Halting Problem. 
Proposition $4.46\left(R C A_{0}\right)$. There exists a computable integral domain $Q$, not Atomic, such that any sequence $\left\langle c_{i}\right\rangle_{i \in \omega}$ from $Q$, with $c_{k+1}$ properly dividing $c_{k}$ for all $k$, computes $\emptyset^{\prime}$.

Proof. Let $Q=Q_{\omega}$. By Proposition 4.35, it is non-Atomic. By Proposition 4.45 , any chain $\left\langle c_{i}\right\rangle_{i \in \omega}$ of $Q$ with $c_{k+1}$ properly dividing $c_{k}$ computes $\emptyset^{\prime}$.

Finally, we obtain our desired result.

Theorem $4.47\left(R C A_{0}\right)$. Theorem 1.3 is equivalent to $A C A_{0}$.

Proof. The proof of Theorem 1.3, carried in $A C A_{0}$ provides the first direction of the equivalence.

Let $M$ be a model of $R C A_{0}+$ Theorem 1.3. Let $X \in M$, we show $X^{\prime}$ exists. Note that $M$ is closed under Turing reducibility. In $M$, from Proposition 4.46 and $X$, obtain an $X$-computable ring $Q$ which is non-Atomic and if $\left\langle c_{i}\right\rangle_{i \in \omega}$ from $Q$ with $\forall k c_{k+1} \mid c_{k}$ and they do not associate then $X \oplus\left\langle c_{i}\right\rangle \geq_{T} X^{\prime}$.

By Theorem 1.3, there is such a sequence in $M$. So $X^{\prime} \in M$. This shows Theorem 1.3 implies $A C A_{0}$.

This concludes our proof. We have shown that Theorem 1.3 is equivalent to $A C A_{0}$, over the base system $R C A_{0}$.

\subsection{Conclusion}

We have investigated the proof-theoretic strengths of Theorems 1.2 and 1.3. We have found that Theorem 1.2 is provable within $R C A_{0}$ and that Theorem 1.3 is equivalent to $A C A_{0}$ over the base system $R C A_{0}$.

The proof of Theorem 1.2 in $R C A_{0}$ is straight-forward and relies on a $\Sigma_{1}$-induction argument. A good open question here would be establishing an equivalence between $R C A_{0}$ and Theorem 1.2 over a weaker base system.

The proof of the equivalence with $A C A_{0}$ was accomplished by proving Theorem 4.46, which gives a non-Atomic integral domain in which any descending chain in divisibility computes $\emptyset^{\prime}$. The conjunction of this result and Theorem 1.3 proves the existence of $\emptyset^{\prime}$ in any $\omega$-model of $R C A_{0}+$ Theorem 1.3. 
One thing to notice here is that the direct proof in $A C A_{0}$ of Theorem 1.3 uses $\emptyset^{\prime \prime}$ as an oracle, so it is natural to ask whether a version of Theorem 4.46 that implies the existence of $\emptyset^{\prime \prime}$ holds. Hence, we state this as a question.

Question 4.48. Does there exist a computable integral domain $Q$, non-Atomic, such that any sequence $\left\langle c_{i}\right\rangle_{i \in \omega}$ from $Q$, with $c_{k+1}$ properly dividing $c_{k}$ for all $k$, computes $\emptyset^{\prime \prime}$ ? 


\section{Bibliography}

[1] D. Bridges and F. Richman, Varieties of Constructive Mathematics. Lon. Math. Soc. Lec. Ser., 97, Cambridge University Press, Cambridge, 1987.

[2] J. Coykendall and M. Zafrullah, AP-domains and unique factorization. J. of Pure and App. Algebra, 189, (2004), 27-35.

[3] C. J. Conidis, Chain Conditions in Computable Rings. Trans. Amer. Math. Soc., 362, (2010), 6523-6550.

[4] R. G. Downey, S. Lempp and J. R. Mileti, Ideals in Computable Rings. J. Algebra, 314, (2007), 872-887.

[5] R. G. Downey and D. R. Hirschfeldt, Algorithmic Randomness and Complexity. Springer-Verlag 2010.

[6] H. M. Friedman, S. G. Simpson and R. L. Smith, Countable Algebra and Set Existence Axioms. Ann. of Pure and App. Logic, 25, (1983), 141-181.

[7] A. Fröhlich and J.C. Sheperdson, On the factorization of polynomials in finite number of steps. Math. Z., 62, (1955), 331-334.

[8] A. Fröhlich and J.C. Sheperdson, Effective procedures in field theory. Philos. Trans. Royal Soc. London Ser. A, 248, (1956), 407-432.

[9] J. A. Gallian, Contemporary Abstract Algebra. Houghton Mifflin Company, Boston, 2nd edition, 2006.

[10] A. Grams, Atomic Rings and the Ascending Chain Condition for Principal Ideals. Proceedings of the Cambridge Philosophical Society, 75, (1974), 321-329.

[11] K. Hatzikiriakou, Commutative algebra in subsystems of second order arithmetic. PhD Thesis, Penn. State University, 1989.

[12] M. Kneser, Bemerkung über die Primpolynomzerlegung in epdlich vielen Schritten. Math. Z., 57, (1953), 238-240. 
[13] S. Lang, Undergraduate Algebra. Undergraduate texts in mathematics, Springer, 2004.

[14] A. I. Mal'cev, Constructive algebra I. Russ. Math. Surv., 16, (1961), 77-129.

[15] G. Metakides and A. Nerode, Effective content of field theory. Ann. Math. Logic, 17, (1979), 289-320.

[16] R. Mines, F. Richman and W. Ruitenburg, A course in constructive algebra. Springer, New York, 1988.

[17] A. Montalbán, Open Questions in Reverse Mathematics. manuscript, 2010.

[18] J. Mott, The Theory of Divisibility. in Factorization in Integral Domains, Marcel Dekker Inc., New York, 1997.

[19] A. Nerode and N. Greenberg The theory of the donut. Elliptic curves for undergraduates. manuscript, 2012.

[20] M. O. Rabin, Computable algebra, general theory and theory of computable fields. Trans. Amer. Math. Soc., 95, (1960), 341-360.

[21] M. O. Rabin, Computable algebraic systems, in Summer Institute for Symbolic Logic, Cornell University. Institute for Defence Analyses, 1957.

[22] J. Rao and S.G. Simpson, Factorization in subsytems of second order arithmetic, quoted in Handbook of Recursive Mathematics. Elsevier, 1998.

[23] S. G. Simpson, Subsystems of Second Order Arithmetic. Cambridge University Press, 2nd edition, 2010.

[24] S. G. Simpson and J. Rao, Reverse Algebra, in Handbook of Recursive Mathematics. Elsevier, 1998.

[25] S. G. Simpson and R. L. Smith, Factorization of polynomials and $\Sigma_{1}^{0}$ Induction. Ann. Pure Appl. Logic, 31, (1986), 289-306.

[26] V. Stoltenberg-Hansen and J. V. Tucker, Computable rings and fields, in Handbook of Computability Theory. Elsevier, 1999.

[27] R. I. Soare, Recursively Enumerable Sets and Degrees. Springer-Verlag, New York, 1987.

[28] D. R. Solomon, Reverse Mathematics and Ordered Groups. PhD Thesis, Cornell University, 1998.

[29] B.L. van der Waerden, Eine Bemerkung über die Unzerlegbarkeit von Polynomen. Math. Ann., 102, (1930), 738-739. 